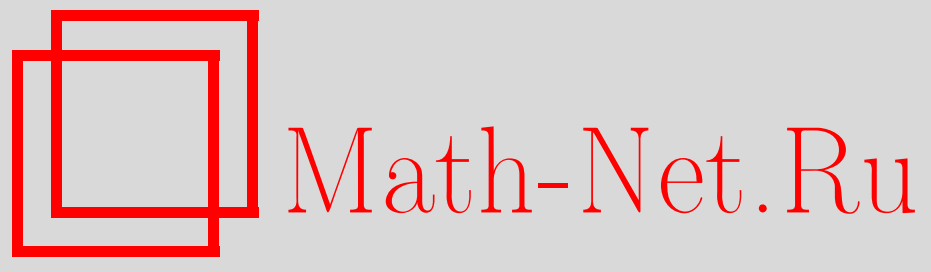

И. К. Бабенко, Алгебра, геометрия и топология группы подстановок формальных степенных рядов, УМН, 2013, том 68 , выпуск 1, 3-76

DOI: https://doi.org/10.4213/rm9471

Использование Общероссийского математического портала Math-Net.Ru подразумевает, что вы прочитали и согласны с пользовательским соглашением http://www . mathnet.ru/rus/agreement

Параметры загрузки:

IP : 54.166 .219 .16

26 апреля 2023 г., 17:45:05

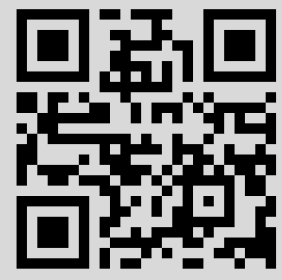




\title{
Алгебра, геометрия и топология группы подстановок формальных степенных рядов
}

\author{
И. К. Бабенко
}

Работа посвящена систематическому описанию различных свойств группы $\mathscr{J}(\mathbf{k})$ формальных степенных рядов от одной переменной с коэффициентами в некотором коммутативном кольце с единицей $\mathbf{k}$. В последние 20 лет эта топологическая группа интенсивно изучалась и был получен ряд интересных результатов о ее строении. В статье обсуждается, каким образом эта группа возникает в ряде проблем из различных областей математики, таких, например, как комплексные кобордизмы или симплектическая топология. Рассматривается, как классические проблемы локальной униформизации и включения ростка голоморфного отображения в поток связаны с общей структурой группы формальных степенных рядов с комплексными коэффициентами.

Библиография: 115 названий.

Ключевые слова: степенные ряды, обратный предел, топологическая группа, про-р-группа.

DOI: $10.4213 / \mathrm{rm} 9471$

\section{СоДЕРЖАНИЕ}

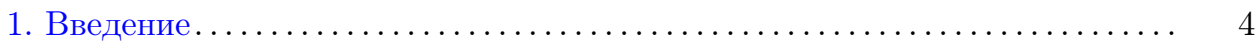

2. Группа $\mathscr{J}(\mathbf{k})$ над коммутативным кольцом с единицей $\mathbf{k} \ldots \ldots \ldots \ldots \ldots$.

2.1. Основные определения и конструкции ................... 9

2.2. Подгруппы, соответствующие подкольцам и идеалам ......... 11

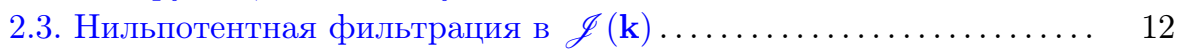

2.4. Обратные пределы топологических групп; топология на $\mathscr{J}(\mathbf{k}) \cdot 13$

2.5. $\mathscr{J}(\mathbf{k})$ как группа автоморфизмов кольца $\mathbf{k}[[x]] \ldots \ldots \ldots \ldots \ldots . . \ldots$

2.6. Однопараметрические подгруппы .................... 16

2.7. Некоторые топологические свойства группы $\mathscr{J}(\mathbf{k}) \ldots \ldots \ldots \ldots . . \ldots 17$

2.8. Группа автоморфизмов группы $\mathscr{J}(\mathbf{k})$ и группа $\mathscr{A}(\mathbf{k}) \ldots \ldots \ldots . .19$

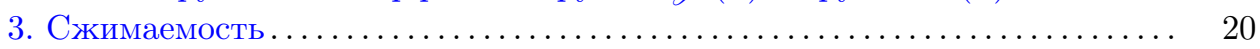

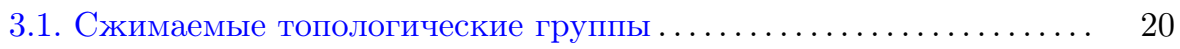

3.2. Одна теорема Эйзенштейна об алгебраических функциях...... 21

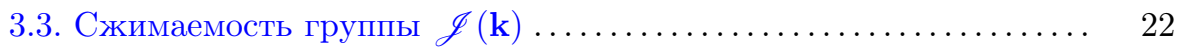

Работа выполнена при поддержке РФФИ (гранты № 10-01-00257-а, 11-01-90413-Укр-ф-а).

(C) И. К. БАБенко, 2013 


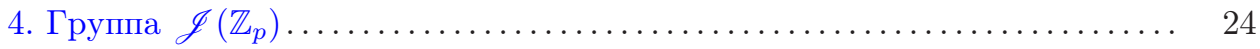

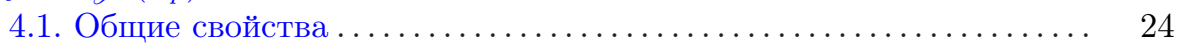

4.2. $p$-группы $\mathscr{J}_{n}(\mathbf{k})$, для кольца характеристики $p \ldots \ldots \ldots \ldots \ldots .25$

4.3. Системы топологических образующих, определяющие соотно-

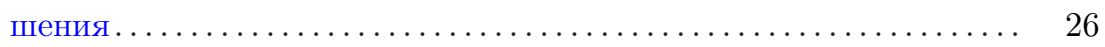

4.4. Универсальность группы $\mathscr{J}\left(\mathbb{Z}_{p}\right) \ldots \ldots \ldots \ldots \ldots \ldots \ldots \ldots \ldots \ldots .28$

4.5. Дальнейшие топологические свойства групп $\mathscr{J}\left(\mathbb{Z}_{p}\right)$ и некоторых

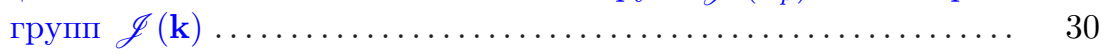

4.6. Индексные и некоторые другие большие подгруппы........... 32

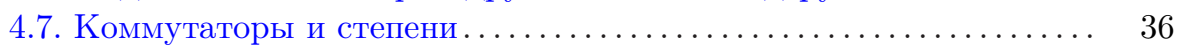

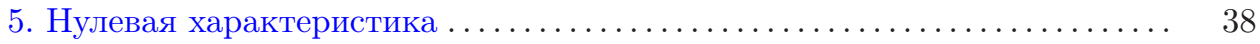

5.1. Некоторые общие свойства....................... 38

5.2. Бесконечномерные группы Ли ..................... 41

5.3. Алгебра Ли........................................ 42

5.4. Экспоненциальное отображение........................ 43

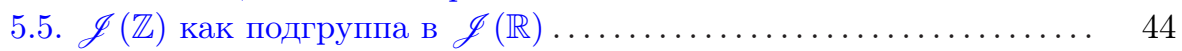

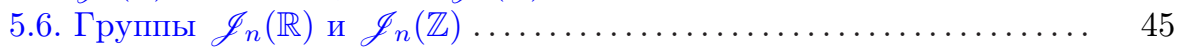

5.7. Симплектические многообразия $\left(K_{2 n}, \Omega_{2 n}\right)$ и произведения Мас-

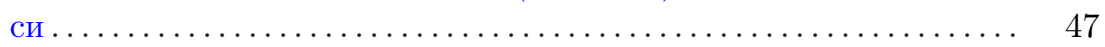

5.8. Вещественные когомологии группы $\mathscr{J}(\mathbb{Z}) \ldots \ldots \ldots \ldots \ldots \ldots \ldots . \ldots 48$

5.9. Несколько замечаний о когомологиях группы $\mathscr{J}_{n}(\mathbb{Z}) \ldots \ldots \ldots . \quad 51$

6. Подгруппы ростков голоморфных диффеоморфизмов в группах $\mathscr{J}(\mathbb{C})$

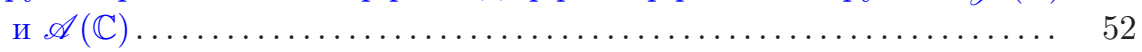

6.1.Однопараметрические подгруппы, классы сопряженных в $\mathscr{A}(\mathbb{C})$ и проблема Зигеля............................. 52

6.2. Однопараметрические подгруппы и классы сопряженных в груп-

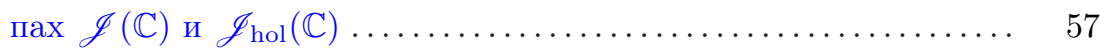

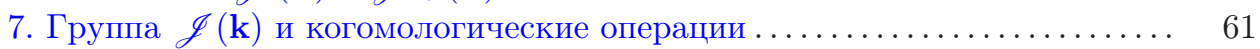

7.1. Инвариантные дифференциальные операторы на группе $\mathscr{J}(\mathbf{k}) .61$

7.2. Когомологические операции ........................ 64

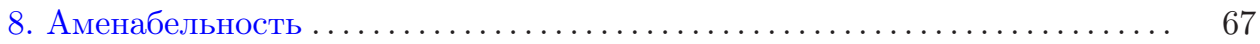

8.1. Несколько замечаний об аменабельности не локально ком-

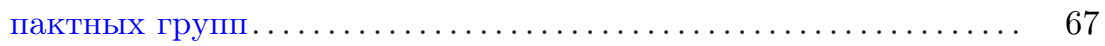

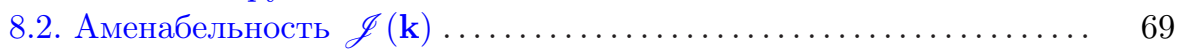

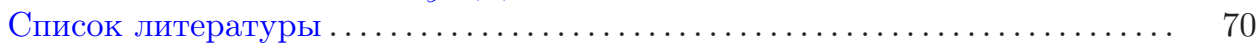

\section{1. Введение}

Пусть $\mathbf{k}$ - коммутативное кольцо. В дальнейшем, если не оговорено противное, мы всегда будем предполагать наличие единицы в кольце k. Рассмотрим множество $\mathscr{J}(\mathbf{k})$ всевозможных формальных степенных рядов от переменной $x$ с коэффициентами из $\mathbf{k}$, имеющих вид:

$$
f(x)=x+\alpha_{1} x^{2}+\alpha_{2} x^{3}+\cdots+\alpha_{n} x^{n+1}+\cdots, \quad \alpha_{n} \in \mathbf{k}, \quad n=1,2, \ldots .
$$

Снабженное операцией подстановки ряда в ряд, множество $\mathscr{J}(\mathbf{k})$ становится группой. Даже для простейшего кольца $\mathbf{k}=\mathbb{Z}_{2}$ группа $\mathscr{J}(\mathbf{k})$ несчетна и ее 
следует рассматривать как топологическую группу. Ниже мы увидим, что для любого основного кольца $\mathbf{k}$ топология на $\mathscr{J}(\mathbf{k})$ играет существенную роль, этот вопрос подробно обсуждается в разделе 2 .

Группа $\mathscr{J}(\mathbf{k})$ обладает рядом замечательных свойств. Часть из них, например, нильпотентная аппроксимируемость, аменабельность и некоторые другие, не зависят от свойств основного кольца. Большинство же алгебраических, да и топологических свойств этой группы определяются свойствами исходного кольца $\mathbf{k}$.

Наверное, первой работой, посвященной группе формальных степенных рядов (даже от многих переменных), была появившаяся в 1950 г. работа Гото [51]. Рассматривая вопрос в слишком общей постановке, он получил в этой работе несколько общих результатов, относящихся к группе формальных степенных рядов от нескольких переменных, которые становятся тривиальными в случае одной переменной. Никакого видимого продолжения эта работа не получила.

Первое многоплановое изучение строения и свойств группы $\mathscr{J}(\mathbf{k})$ было предпринято Дженнингсом [59] почти 60 лет назад. И сейчас эту статью без преувеличения можно порекомендовать как лучшее введение в предмет. $\mathrm{K}$ сожалению, эта работа сразу не получила никакого развития и вскорости была забыта, часть ее результатов впоследствии была заново доказана, часто без надлежащих ссылок, другими авторами.

Раздел 2 этого обзора содержит общую информацию о группе $\mathscr{J}(\mathbf{k})$, его начало во многом основано на работе [59]. Этот раздел написан несколько более подробно, чем остальные, часть утверждений сопровождается набросками доказательств. Это сделано для того, чтобы дать читателю представление об идеях и методах, используемых в доказательствах, и облегчить чтение текста начинающим исследователям.

В течение более чем 30 лет после статьи [59] фактически не было работ, где изучалась бы группа $\mathscr{J}(\mathbf{k})$. Наверное, единственное исключение - работа Макенхоупта [82], в которой исследовалась группа автоморфизмов группы $\mathscr{J}(\mathbf{k})$.

Группа $\mathscr{J}(\mathbf{k})$ снова привлекла внимание алгебраистов в 1988 г. - после появления работы Джонсона [60]. Эта статья, практически не содержащая новых, по сравнению с [59], результатов, сыграла существенную роль в деле пропаганды исследований группы $\mathscr{J}(\mathbf{k})$. С начала 90 -х годов прошлого века систематически стали появляться работы, посвященные группе $\mathscr{J}(\mathbf{k})$, а к середине 90-х поток работ в этом направлении стал значительным. Практически все работы этого периода касались случая конечного поля $\mathbf{k}=\mathbb{F}_{q}$ характеристики $p$, содержащего $q=p^{d}$ элементов. Часть работ относилась к "совсем частному случаю" группы $\mathscr{J}\left(\mathbb{Z}_{p}\right)$, где $\mathbb{Z}_{p}=\mathbb{Z} / p \mathbb{Z}$ - простое поле из $p$ элементов. Такой интерес именно к группе $\mathscr{J}\left(\mathbb{Z}_{p}\right)$ совсем не случаен. С одной стороны, группа $\mathscr{J}\left(\mathbb{Z}_{p}\right)$ является естественным примером про-р-группы и уже поэтому заслуживает пристального изучения, с другой стороны, она связана с рядом вопросов теории чисел, в частности, с теорией Галуа локальных полей.

Активное изучение группы $\mathscr{J}\left(\mathbb{Z}_{p}\right)$ в 90-е годы прошлого и начале нынешнего века позволило ответить на целый ряд вопросов общей теории про-р-групп. Например, оказалось, что сама $\mathscr{J}\left(\mathbb{Z}_{p}\right)$ является универсальной группой в классе про- $p$-групп со счетной базой. Это означает, что любая про- $p$-группа со счетной 
базой вкладывается в $\mathscr{J}\left(\mathbb{Z}_{p}\right)$ как замкнутая подгруппа. Хотя универсальные про- $p$-группы были известны и раньше, универсальность $\mathscr{J}\left(\mathbb{Z}_{p}\right)$ представляет особый интерес в силу естественности этой группы.

Начиная с работы [60] многие исследования группы $\mathscr{J}\left(\mathbb{Z}_{p}\right)$ в 90-х годах были проведены в Ноттингемском университете, и в тот же период за группой $\mathscr{J}\left(\mathbb{Z}_{p}\right)$ закрепился термин Ноттингемская группа. Исследования группы $\mathscr{J}\left(\mathbb{Z}_{p}\right)$ в последние 20 лет отражены в разделе 4 настоящего обзора. Отметим, что в этом направлении имеется еще обзор Камины [25], который, однако, не охватывает период после 2000 г.

Для конечного кольца $\mathbf{k}$ группа $\mathscr{J}(\mathbf{k})$ является проконечной группой и гомеоморфна канторову множеству как топологическое пространство. Гораздо более сложная топологическая структура возникает на $\mathscr{J}(\mathbf{k})$ в случае бесконечного основного кольца $\mathbf{k}$. Так, если $\mathbf{k}$ бесконечно и дискретно, например $\mathbf{k}=\mathbb{Z}$, то $\mathscr{J}(\mathbf{k})$ уже не локально компактна и даже не вкладывается ни в какую локально компактную группу. Здесь алгебраические и топологические свойства $\mathscr{J}(\mathbf{k})$ сильно переплетаются. Например, если кольцо k содержит поле рациональных чисел $\mathbb{Q}$, то при любой топологии основного кольца $\mathbf{k}$ любой непрерывный гомоморфизм $\mathscr{J}(\mathbf{k})$ в локально компактную группу имеет нетривиальное ядро. Этот круг вопросов обсуждается в п. 2.7.

Еще одним общим топологическим свойством групп $\mathscr{J}(\mathbf{k})$ является разновидность самоподобия, называемая в дальнейшем сжимаемостью. Сжимаемыми оказываются группы $\mathscr{J}(\mathbf{k})$ для практически всех "разумных" колец $\mathbf{k}$. Здесь топологическую группу мы называем сжимаемой, если она вкладывается в любую наперед заданную окрестность своей единицы. Сжимаемость напоминает свойство фрактальности и может возникать не только когда группа $\mathscr{J}(\mathbf{k})$ нульмерна, но и в любой размерности. Конструкция сжимаемости для групп $\mathscr{J}(\mathbf{k})$ опирается на классическую лемму, восходящую к Эйзенштейну, об алгебраических функциях, разлагающихся в ряд с рациональными коэффициентами. Свойству сжимаемости посвящен третий раздел.

Характеристика 0 представляет немалый интерес сразу по нескольким причинам. Как группа, наибольший интерес представляет, конечно, $\mathscr{J}(\mathbb{Z})$, которая до недавнего времени пристально не изучалась. Группа $\mathscr{J}(\mathbb{Z})$ играет роль равномерной решетки в бесконечномерной группе Ли $\mathscr{J}(\mathbb{R})$, смоделированной на локально выпуклом топологическом векторном пространстве $\mathbb{R}^{\infty}$. Подгруппа $\mathscr{J}(\mathbb{Z})$ кокомпактна в $\mathscr{J}(\mathbb{R})$, но не дискретна. Это объясняет достаточно сложную структуру классифицирующего пространства $K_{\infty}=\mathscr{J}(\mathbb{R}) / \mathscr{J}(\mathbb{Z})$, которое, например, гомотопически не эквивалентно никакому $C W$-комплексу и не имеет универсального накрытия (п. 5.5).

Хотя $\mathscr{J}(\mathbb{Z})$ имеет четыре образующие как топологическая группа (п. 5.1), ее строение достаточно сложно, о чем свидетельствуют ее вещественные когомологии. В силу локальной некомпактности $\mathscr{J}(\mathbb{Z})$ подходящей теорией когомологий групп являются когомологии с так называемыми локально постоянными носителями (п. 5.8). Такие когомологии группы $\mathscr{J}(\mathbb{Z})$ оказываются изоморфными вещественным когомологиям Александера-Чеха пространства $K_{\infty}$, которые, в свою очередь, изоморфны вещественным когомологиям нильпотентной 
части алгебры формальных векторных полей на прямой. Этот и многие другие близкие вопросы обсуждаются в разделе 5 .

Другая причина, по которой случай колец $\mathbf{k}$ нулевой характеристики представляет интерес, состоит в различных приложениях групп $\mathscr{J}(\mathbf{k})$ в геометрии и топологии. Так, конечномерные редукции групп $\mathscr{J}(\mathbb{R})$ и $\mathscr{J}(\mathbb{Z})$ приводят к группам $\mathscr{J}_{n}(\mathbb{R})$ и $\mathscr{J}_{n}(\mathbb{Z})$ полиномов степени не выше $n+1$ соответственно с вещественными и целыми коэффициентами. Нильпотентная группа Ли $\mathscr{J}_{n}(\mathbb{R})$ обладает инвариантной симплектической или контактной структурой в зависимости от четности $n$. Это обстоятельство позволяет, например, строить компактные симплектические многообразия, обладающие нетривиальными произведениями Масси сколь угодно большого веса и, следовательно, очень сильно отличающиеся от кэлеровых многообразий (п. 5.7).

Еще один круг приложений групп $\mathscr{J}(\mathbb{R})$ и $\mathscr{J}(\mathbb{Z})$ был найден в 70 -е годы Бухштабером и Шокуровым [22] еще до систематического изучения этих групп. Бесконечномерная группа Ли $\mathscr{J}(\mathbb{R})$ имеет единственную координатную карту, где естественными координатами служат коэффициенты ряда (1), так называемые канонические координаты. Это подсказывает, что в качестве естественного пространства функций на $\mathscr{J}(\mathbb{R})$ можно рассматривать пространство полиномов от канонических координат. Алгебра левоинвариантных дифференциальных операторов на группе $\mathscr{J}(\mathbb{R})$ представляет отдельный интерес, так как она изоморфна универсальной обертывающей алгебре нильпотентной части алгебры формальных векторных полей на прямой. В алгебре всех инвариантных операторов имеется подалгебра целочисленных инвариантных дифференциальных операторов, т. е. тех операторов, которые оставляют инвариантным подмодуль полиномов с целыми коэффициентами. В [22] было показано, что алгебра целочисленных инвариантных операторов изоморфна хорошо известной в топологии алгебре Ландвебера-Новикова. Эта последняя является подалгеброй стабильных когомологических операций в комплексных кобордизмах, а всю алгебру стабильных операций можно рассматривать как модуль над алгеброй Ландвебера-Новикова. Этот круг вопросов рассматривается в разделе 7.

Наконец, еще одно замечательное явление, возникающее в нулевой характеристике, состоит в следующем. Группа формальных степенных рядов с комплексными коэффициентами $\mathscr{J}(\mathbb{C})$, а также полная группа таких рядов $\mathscr{A}(\mathbb{C})$ (где первый коэффициент - любое ненулевое комплексное число) содержат естественные подгруппы $\mathscr{J}_{\text {hol }}(\mathbb{C})$ и $\mathscr{A}_{\mathrm{hol}}(\mathbb{C})$, состоящие из рядов с ненулевым радиусом сходимости. Изучение строения групп $\mathscr{J}_{\mathrm{hol}}(\mathbb{C})$ и $\mathscr{A}_{\mathrm{hol}}(\mathbb{C})$, а также структуры их вложения в группы $\mathscr{J}(\mathbb{C})$ и $\mathscr{A}(\mathbb{C})$ соответственно представляет собой крайне сложные и еще очень далекие от полного решения задачи. Можно смело сказать, что исследования, относящиеся к этой тематике, ведутся по крайней мере с 80-х годов XIX века. На протяжении этого периода в многочисленных работах по анализу изучались свойства заданного голоморфного в нуле отображения вида $f(x)=\alpha x+\alpha_{1} x^{2}+\alpha_{2} x^{3}+\cdots, \alpha \neq 0$, с точки зрения возможности его локальной линеаризации или его включения в непрерывную однопараметрическую подгруппу голоморфных отображений. Под линеаризацией (униформизацией) здесь понимается отыскание (если это возможно) такой голоморфной замены координат $x=\phi(z)$, что $g(z)=\phi^{-1}(f(\phi(z)))=\alpha z$. Соот- 
ветственно, под включением в непрерывную подгруппу понимается отыскание (если это возможно) такого непрерывного по $\lambda \in \mathbb{C}$ семейства голоморфных отображений $f_{\lambda}(x)$, что $f_{1}(x)=f(x)$ и $f_{\mu}\left(f_{\lambda}(x)\right)=f_{\lambda+\mu}(x)$. Как выяснилось впоследствии, обе эти задачи разрешимы далеко не всегда. Количество работ по указанной тематике за последние 130 лет огромно, среди наиболее значительных следует отметить следующие: Кёнигс [68], Кремер [28], Зигель [99], Брюно [17], [18] и Йокоз [113], [114], а также Эрдёш и Жаботинский [40], Жаботинский [57], [58], Бейкер [9], [10], Экаль [35]-[37] и Воронин [108].

Классы сопряженных и однопараметрические подгруппы в $\mathscr{J}(\mathbb{C})$ и $\mathscr{A}(\mathbb{C})$ устроены достаточно просто и могут быть легко описаны. Ситуация неимоверно усложняется в голоморфном случае. Результаты цитированных выше исследований, сформулированные на языке теории групп, дают почти полную информацию о классах сопряженных и однопараметрических подгруппах в группах $\mathscr{J}_{\text {hol }}(\mathbb{C})$ и $\mathscr{A}_{\text {hol }}(\mathbb{C})$. Эти результаты также дают определенную информацию о структуре вложений $\mathscr{J}_{\mathrm{hol}}(\mathbb{C}) \subset \mathscr{J}(\mathbb{C})$ и $\mathscr{A}_{\mathrm{hol}}(\mathbb{C}) \subset \mathscr{A}(\mathbb{C})$. Обсуждению этой тематики посвящен раздел 6.

В последнем разделе 8 мы рассматриваем аменабельность и некоторые динамические свойства группы $\mathscr{J}(\mathbf{k})$. В классических работах 20-30-х годов прошлого века, принадлежащих фон Нейману [107] и Боголюбову [16], под аменабельностью некоторой топологической группы $G$ понималось существование левоинвариантного среднего на пространстве ограниченных непрерывных функций, заданных на $G$.

Общая теория инвариантных средних на локально компактных и, в частности, на дискретных группах достаточно хорошо отражена в ставшей уже классической монографии [53]. Удачным дополнением к [53] может служить монография [89].

Поскольку для конечных колец группа $\mathscr{J}(\mathbf{k})$ компактна, то вопрос о существовании инвариантных средних становится интересным лишь в случае бесконечных колец $\mathbf{k}$. Вместе с тем для бесконечного кольца $\mathbf{k}$ группа $\mathscr{J}(\mathbf{k})$ может не быть локально компактной и даже не вкладываться в локально компактные группы. Поэтому хорошо развитая в локально компактном случае теория инвариантных средних к группе $\mathscr{J}(\mathbf{k})$ применена быть не может. Отметим еще одно обстоятельство - группа $\mathscr{J}(\mathbf{k})$ содержит свободные подгруппы для любых колец $\mathbf{k}$, поэтому инвариантное среднее заведомо отсутствует на пространстве всех ограниченных функций.

Оказывается, что инвариантное среднее существует на пространстве ограниченных равномерно непрерывных на $\mathscr{J}(\mathbf{k})$ функций. Это, в свою очередь, влечет существование инвариантной вероятностной меры для непрерывного действия $\mathscr{J}(\mathbf{k})$ на любом компакте. В качестве примера обсуждается естественная борелевская вероятностная мера на бесконечномерном компакте правых смежных классов $K_{\infty}=\mathscr{J}(\mathbb{R}) / \mathscr{J}(\mathbb{Z})$, инвариантная относительно левого действия $\mathscr{J}(\mathbb{R})$.

Наконец, сделаем несколько замечаний, касающихся обозначений. Мы придерживаемся топологической традиции в обозначениях, простое поле из $p$ элементов далее обозначается $\mathbb{Z}_{p}$. При этом конечное поле характеристики $p$, содержащее $q=p^{d}$ элементов, обозначается $\mathbb{F}_{q}$. Кольцо целых $p$-адических чисел 
и поле $p$-адических чисел обозначаются $\mathbb{Z}_{(p)}$ и $\mathbb{Q}_{(p)}$ соответственно. Остальные обозначения не должны вызвать недоразумений.

Как уже видно из введения, группа $\mathscr{J}(\mathbf{k})$ связана со многими весьма далекими друг от друга разделами математики. Ввиду очень большого тематического разнообразия, мы отбирали лишь результаты, связанные либо непосредственно с изучением $\mathscr{J}(\mathbf{k})$, ее подгрупп или факторгрупп, либо с прямыми приложениями этой группы.

\section{2. Группа $\mathscr{J}(\mathbf{k})$ над коммутативным кольцом с единицей $\mathrm{k}$}

Этот раздел посвящен самым общим свойствам группы $\mathscr{J}(\mathbf{k})$, которые фактически не зависят от свойств основного кольца $\mathbf{k}$.

2.1. Основные определения и конструкции. С каждым формальным рядом $f(x)$ вида (1)

$$
f(x)=x+\alpha_{1} x^{2}+\alpha_{2} x^{3}+\cdots=x\left(1+\alpha_{1} x+\alpha_{2} x^{2}+\cdots\right), \quad \alpha_{n} \in \mathbf{k},
$$

можно однозначно связать бесконечную последовательность его коэффициентов

$$
\alpha=\left(\alpha_{1}, \alpha_{2}, \ldots, \alpha_{n}, \ldots\right) \in \mathbf{k}^{\mathbb{N}},
$$

которые далее мы будем называть каноническими координатами на множестве $\mathscr{J}(\mathbf{k})$.

Снабдим $\mathscr{J}(\mathbf{k})$ операцией подстановки ряда в ряд с последующим раскрытием скобок и приведением подобных членов (см. [59]):

$$
g \circ f(x)=g(f(x))=x\left(1+\sum_{i \geqslant 1} \gamma_{i} x^{i}\right),
$$

где

$$
f(x)=x\left(1+\sum_{i \geqslant 1} \alpha_{i} x^{i}\right), \quad g(x)=x\left(1+\sum_{i \geqslant 1} \beta_{i} x^{i}\right)
$$

- два элемента из $\mathscr{J}(\mathbf{k})$. Последовательно выполняя возведения в степень и приведение подобных членов в (3), с помощью рутинных вычислений находим следующие выражения для канонических координат $\gamma_{i}$ :

$$
\begin{gathered}
\gamma_{1}=\alpha_{1}+\beta_{1}, \\
\gamma_{2}=\alpha_{2}+\beta_{2}+2 \alpha_{1} \beta_{1}, \\
\gamma_{3}=\alpha_{3}+\beta_{3}+3 \beta_{2} \alpha_{1}+2 \beta_{1} \alpha_{2}+\beta_{1} \alpha_{1}^{2}, \\
\ldots \ldots \ldots \ldots \ldots \ldots \ldots \ldots \ldots \ldots \ldots \ldots \ldots \ldots \ldots \ldots \ldots \ldots \ldots \\
\gamma_{n}=\alpha_{n}+\beta_{n}+\sum_{s=1}^{n-1} \beta_{s} \phi_{n-s}\left(\alpha_{1}, \alpha_{2}, \ldots, \alpha_{n-s}\right) .
\end{gathered}
$$

Здесь $\phi_{r}\left(\alpha_{1}, \alpha_{2}, \ldots, \alpha_{r}\right)$ - универсальные однородные многочлены мультистепени $r$ с целыми коэффициентами. Это означает, что при умножении переменных $\alpha_{i}$ на соответствующий множитель $t^{i}$ многочлен $\phi_{r}\left(\alpha_{1}, \alpha_{2}, \ldots, \alpha_{r}\right)$ умножится на $t^{r}$. 
ПреДЛОЖЕНИЕ 2.1. Множество формалъных степенных рядов $\mathscr{J}(\mathbf{k})$, снабженное операцией подстановки (3), является группой.

ДокАзАтельство. Отсылая за деталями к [59], кратко наметим доказательство, которое проясняет структуру этой группы. Легко видеть, что ряд $f(x)=x$ является единицей группы $\mathscr{J}(\mathbf{k})$. Далее, заметим, что любой элемент $f(x) \in \mathscr{J}(\mathbf{k})$ определяет некоторое отображение множества $\mathscr{J}(\mathbf{k})$ в себя (перестановку) по правилу $F: x \mapsto f(x)$, т. е. $F: r(x) \mapsto r(f(x))$. Иначе говоря, перестановка $F$ действует на ряды заменой независимой переменной $x$ на ряд $f(x)$. Если теперь $G: x \mapsto g(x)$ - некоторое другое отображение, то композиция отображений $G F: x \mapsto g(f(x))$ соответствует операции подстановки (3). Ассоциативность произведения в $\mathscr{J}(\mathbf{k})$ непосредственно вытекает из закона ассоциативности в группе подстановок на некотором множестве.

Для доказательства существования обратного для $f(x) \in \mathscr{J}(\mathbf{k})$ положим $\gamma_{i}=0, i=1,2, \ldots$, в $(4), n=1,2, \ldots$, и попытаемся рекуррентным образом найти $\beta_{i}$ как функции от $\alpha_{1}, \alpha_{2}, \ldots, \alpha_{i}$. Структура формул $\left(4_{1}\right)-\left(4_{n}\right)$ такова, что это всегда возможно и дает элемент $f^{-1}(x)=x\left(1+\tilde{\alpha}_{1} x^{1}+\tilde{\alpha}_{2} x^{2}+\cdots\right)$. Индуктивно находя коэффициенты $f^{-1}(x)$, мы получаем

$$
\tilde{\alpha}_{n}=-\alpha_{n}+\psi_{n}\left(\alpha_{1}, \alpha_{2}, \ldots, \alpha_{n-1}\right), \quad n=1,2, \ldots,
$$

где $\psi_{n}\left(\alpha_{1}, \alpha_{2}, \ldots, \alpha_{n-1}\right)$ - универсальные однородные многочлены мультистепени $n$ с целыми коэффициентами.

Заметим, что для всех трех элементов $f(x), g(x)$ и $g \circ f(x)$ в формулах $\left(4_{1}\right)-\left(4_{n}\right)$ участвуют лишь первые $n$ коэффициентов. Более детальную структуру многочленов $\phi_{r}$, полезную при тонких вычислениях в группе $\mathscr{J}(\mathbf{k})$, можно найти в [54].

ЗАмечАниЕ 2.2. Несложно показать, что формулы $\left(4_{k}\right), k=1,2, \ldots, n$, определяют структуру группы на прямом произведении $\mathbf{k}^{n}$. Эта группа далее обозначается $\mathscr{J}_{n}(\mathbf{k})$. Очевидно, что $\mathscr{J}_{1}(\mathbf{k})$ - абелева группа, которая изоморфна аддитивной группе кольца $\mathbf{k}$. Группа $\mathscr{J}_{2}(\mathbf{k})$ изоморфна аддитивной группе $\mathbf{k} \oplus \mathbf{k}$. При этом соответствующий изоморфизм нелинейно зависит от канонических координат $\left(\alpha_{1}, \alpha_{2}\right)$. Найти явный вид этого изоморфизма мы оставляем читателю в качестве упражнения.

Оказывается, что $\mathscr{J}_{3}(\mathbf{k})$ изоморфна группе Гейзенберга верхнетреугольных матриц вида

$$
\left(\begin{array}{ccc}
1 & u & w \\
0 & 1 & v \\
0 & 0 & 1
\end{array}\right), \quad \text { где } \quad u, v, w \in \mathbf{k}
$$

над кольцом k. Рекомендуем читателю найти явное выражение для такого изоморфизма, при этом координаты $u, v, w$ будут нелинейно зависеть от $\alpha_{1}, \alpha_{2}, \alpha_{3}$.

Иногда удобно $n$-й коэффициент элемента $f(x) \in \mathscr{J}(\mathbf{k})$ вида (1) обозначать $\alpha_{n}(f(x))$. Величину

$$
D(f(x))=\inf _{\alpha_{n}(f) \neq 0} n
$$


называют глубиной элемента $f(x)$, считая при этом, что $D(x)=\infty$. Везде далее под $\mathscr{O}\left(x^{n}\right)$ понимается некоторый формальный ряд, содержащий $x$ в степенях не меньше $n$.

Для вычислений в группе $\mathscr{J}(\mathbf{k})$ оказывается полезным следующее утверждение.

ЛЕмма 2.3. Для двух элементов $f(x)=x\left(1+\alpha_{r} x^{r}\right)+\mathscr{O}\left(x^{r+2}\right), g(x)=$ $x\left(1+\beta_{s} x^{s}\right)+\mathscr{O}\left(x^{s+2}\right)$ группь $\mathscr{J}(\mathbf{k})$ и произвольного целого $k$ справедливы формуль:

a) $f^{g}=g^{-1} \circ f \circ g(x)=x\left(1+\alpha_{r} x^{r}\right)+\mathscr{O}\left(x^{r+2}\right)$;

b) $[f, g]=f^{-1} \circ g^{-1} \circ f \circ g(x)=x\left(1+(r-s) \alpha_{r} b_{s} x^{r+s}\right)+\mathscr{O}\left(x^{r+s+2}\right)$;

c) $f^{k}=x\left(1+k \alpha_{r} x^{r}\right)+\mathscr{O}\left(x^{r+2}\right)$.

ДокАзАТЕльство. Лемма доказывается прямыми вычислениями. Так, например, для доказательства а) сначала заметим, что если $s>r$, то формула очевидна, поскольку $f \circ g(x)=x\left(1+\alpha_{r} x^{r}\right)+\mathscr{O}\left(x^{r+2}\right)=g \circ f(x)+\mathscr{O}\left(x^{r+2}\right)$. Предположим, что $s \leqslant r$, тогда

$$
g(x)=x\left(1+\sum_{i=s}^{r} \beta_{i} x^{i}\right)+\mathscr{O}\left(x^{r+2}\right) .
$$

Из формул (4) получаем

$$
f \circ g(x)=x\left(1+\sum_{i=s}^{r} \beta_{i} x^{i}+\alpha_{r} x^{r}\right)+\mathscr{O}\left(x^{r+2}\right)=g \circ f(x)+\mathscr{O}\left(x^{r+2}\right),
$$

откуда вытекает результат.

Доказательство b) мы оставляем читателю в качестве полезного упражнения. Для доказательства с) в случае положительного $k$ последовательно применяем формулы (4) к $f^{2}=f \circ f, f^{3}=f^{2} \circ f$ и т. д. В случае отрицательного $k$ применяем формулу (5) к уже вычисленному $f^{-k}$.

2.2. Подгруппы, соответствующие подкольцам и идеалам. Данное в начале работы определение группы $\mathscr{J}(\mathbf{k})$ остается справедливым и для колец $\mathbf{k}$, не имеющих единицы. Более того, при работе с подкольцами и идеалами в $\mathbf{k}$ даже удобно предполагать возможное отсутствие единицы в основном кольце.

Рассмотрим некоторое подкольцо $\mathbf{s} \subset \mathbf{k}$; очевидно, что $\mathscr{J}(\mathbf{s})$ является подгруппой $\mathscr{J}(\mathbf{k})$. Если $\mathbf{s} \subset \mathbf{k}$ - идеал, то будем говорить, что элементы $f(x)$, $g(x) \in \mathscr{J}(\mathbf{k})$ являются s-эквивалентными, и писать $f(x) \equiv g(x) \bmod \mathbf{s}$, если $\alpha_{n}-\beta_{n} \in \mathbf{s}, n=1,2, \ldots$ Из формул (4) вытекает, что для любых $f \in \mathscr{J}(\mathbf{k})$ и $g \in \mathscr{J}(\mathbf{s})$

$$
f \circ g \equiv g \circ f \equiv f \quad \bmod \mathbf{s} .
$$

Это, в свою очередь, влечет

$$
f^{-1} \circ g \circ f \equiv x \quad \bmod \mathbf{s} .
$$

Последняя эквивалентность означает, что $\mathscr{J}(\mathbf{s})$ - нормальная подгруппа в $\mathscr{J}(\mathbf{k})$. Положим $\overline{\mathbf{k}}=\mathbf{k} / \mathbf{s}$ и пусть $\theta: \mathbf{k} \rightarrow \overline{\mathbf{k}}-$ естественная проекция. Определим 
отображение множеств $\Theta: \mathscr{J}(\mathbf{k}) \rightarrow \mathscr{J}(\overline{\mathbf{k}})$ формулой

$$
\Theta(f(x))=x\left(1+\theta\left(\alpha_{1}\right) x+\theta\left(\alpha_{2}\right) x^{2}+\cdots\right) .
$$

Используя формулы (4), легко видеть, что это отображение согласовано с произведениями и, следовательно, является эпиморфизмом групп. Также очевидно, что $\operatorname{ker} \Theta=\mathscr{J}(\mathbf{s})$, откуда вытекает следующее равенство:

$$
\mathscr{J}(\overline{\mathbf{k}})=\mathscr{J}(\mathbf{k}) / \mathscr{J}(\mathbf{s})
$$

Пусть $\mathbf{s}$ и $\mathbf{t}-$ два взаимно аннулирующих подкольца в $\mathbf{k}$ (для любых $a \in \mathbf{s}$ и $b \in \mathbf{t}$ выполнено равенство $a b=0$ ). Несложно показать, что соответствующие подгруппы $\mathscr{J}(\mathbf{s})$ и $\mathscr{J}(\mathbf{t})$ поэлементно коммутируют. Если, кроме того, $\mathbf{k}=$ $\mathbf{s} \oplus \mathbf{t}$, то

$$
\mathscr{J}(\mathbf{k})=\mathscr{J}(\mathbf{s}) \times \mathscr{J}(\mathbf{t}) .
$$

Из вышеприведенных рассуждений вытекает следующее предложение.

ПреДЛОЖЕНИЕ 2.4. а) Для любого подколъца $\mathbf{s} \subset \mathbf{k}$ группа $\mathscr{J}(\mathbf{s})$ является подгруппой $\mathscr{J}(\mathbf{k})$.

b) Если $\mathbf{s} \subset \mathbf{k}-$ идеал, то подгруппа $\mathscr{J}(\mathbf{s})$ нормальна в $\mathscr{J}(\mathbf{k})$ и имеется естественный изоморфизм

$$
\mathscr{J}(\mathbf{k} / \mathbf{s}) \simeq \mathscr{J}(\mathbf{k}) / \mathscr{J}(\mathbf{s}) .
$$

c) $Е с л и \mathbf{k}=\mathbf{s} \oplus \mathbf{t}$, где $\mathbf{s}$ и $\mathbf{t}-$ два взаимно аннулирующих подкольца, то

$$
\mathscr{J}(\mathbf{k})=\mathscr{J}(\mathbf{s}) \times \mathscr{J}(\mathbf{t}) .
$$

2.3. Нильпотентная фильтрация в $\mathscr{J}(\mathbf{k})$. Рассмотрим следующие подмножества в $\mathscr{J}(\mathbf{k})$ :

$$
\begin{aligned}
& \mathscr{J}^{n}(\mathbf{k})=\left\{f(x) \in \mathscr{J}(\mathbf{k}) \mid \alpha_{1}=\alpha_{2}=\cdots=\alpha_{n-1}=0\right\}, \quad n=2,3, \ldots, \\
& \mathscr{J}^{1}(\mathbf{k})=\mathscr{J}(\mathbf{k}) .
\end{aligned}
$$

Из леммы 2.3, а) вытекает, что $\mathscr{J}^{n}(\mathbf{k}), n=1,2, \ldots$, являются нормальными подгруппами в $\mathscr{J}(\mathbf{k})$, а из части b) той же леммы получаем, что

$$
\left[\mathscr{J}^{n}(\mathbf{k}), \mathscr{J}^{m}(\mathbf{k})\right] \subseteq \mathscr{J}^{n+m}(\mathbf{k}), \quad \gamma_{n}(\mathscr{J}(\mathbf{k})) \subseteq \mathscr{J}^{n+1}(\mathbf{k}) \quad \text { и } \quad[\mathscr{J}(\mathbf{k})]_{n} \subseteq \mathscr{J}^{2^{n}}(\mathbf{k}),
$$

где $\gamma_{n}(\mathscr{J}(\mathbf{k}))=\left[\mathscr{J}(\mathbf{k}), \gamma_{n-1}(\mathscr{J}(\mathbf{k}))\right]-n$-й член нижнего центрального ряда, $\mathrm{a}[\mathscr{J}(\mathbf{k})]_{n}=\left[[\mathscr{J}(\mathbf{k})]_{n-1},[\mathscr{J}(\mathbf{k})]_{n-1}\right]-n$-й коммутант группы $\mathscr{J}(\mathbf{k})$.

Нормальные подгруппы $\mathscr{J}^{n}(\mathbf{k})$ играют важную роль в изучении самой группы $\mathscr{J}(\mathbf{k})$. Соответствующие факторгруппы естественно изоморфны группам $\mathscr{J}_{n-1}(\mathbf{k})$, уже встречавшимся в замечании 2.2 , т. е.

$$
\mathscr{J}_{n}(\mathbf{k})=\mathscr{J}(\mathbf{k}) / \mathscr{J}^{n+1}(\mathbf{k}), \quad n=1,2, \ldots
$$

Из включений (6) вытекает, что все эти факторгруппы нильпотентны. Группы $\mathscr{J}_{n}(\mathbf{k}), n=1,2, \ldots$, естественно интерпретировать как группы полиномов

$$
\mathscr{J}_{n}(\mathbf{k})=\left\{P(x)=x\left(1+\alpha_{1} x+\alpha_{2} x^{2}+\cdots+\alpha_{n} x^{n}\right)\right\}
$$


а операцию - как подстановку полинома в полином с последующим отбрасыванием всех членов в степенях $n+2$ и выше:

$$
P \circ Q(x)=P(Q(x)) \bmod \mathscr{O}\left(x^{n+2}\right) .
$$

Подгруппы $\left\{\mathscr{J}^{n}(\mathbf{k})\right\}_{n=1}^{\infty}$ имеют в пересечении лишь единичный элемент:

$$
\bigcap_{n=1}^{\infty} \mathscr{J}^{n}(\mathbf{k})=x \in \mathscr{J}(\mathbf{k}) .
$$

Для нижнего центрального ряда

$$
\mathscr{J}(\mathbf{k}) \supseteq[\mathscr{J}(\mathbf{k}), \mathscr{J}(\mathbf{k})]=\gamma_{2}(\mathscr{J}(\mathbf{k})) \supseteq \cdots \supseteq \gamma_{n}(\mathscr{J}(\mathbf{k})) \supseteq \cdots
$$

из формул (6) и (7) вытекает, что

$$
\bigcap_{n=1}^{\infty} \gamma_{n}(\mathscr{J}(\mathbf{k}))=x .
$$

Таким образом, мы приходим к выводу, что $\mathscr{J}(\mathbf{k})$ - остаточно нильпотентная группа.

ЗАмечАние 2.5. Свойства подгрупп $\left\{\mathscr{J}^{n}(\mathbf{k})\right\}_{n=1}^{\infty}$ зависят от алгебраических свойств основного кольца $\mathbf{k}$. Так, например, если $\mathbf{k}-$ поле характеристики, отличной от 2 и 3 , то $\left\{\mathscr{J}^{n}(\mathbf{k})\right\}_{n=1}^{\infty}$ будут характеристическими подгруппами B $\mathscr{J}(\mathbf{k})$.

Свойство (7) позволяет ввести топологию на $\mathscr{J}(\mathbf{k})$. С этой целью подмножества $\left\{\mathscr{J}^{n}(\mathbf{k})\right\}_{n=1}^{\infty}$ объявляются базой окрестностей единицы $x$, а затем это семейство открытых множеств продолжается до базы топологии на $\mathscr{J}(\mathbf{k})$ с помощью левых и правых действий.

Так полученная топология является топологией формальной сходимости (ФС-топология). Легко видеть, что сходимость в этой топологии означает, что последовательность $\left\{f_{k}(x)\right\}_{k=1}^{\infty}$ стабилизируется modulo $\mathscr{O}\left(x^{n}\right)$, если $n$ стремится к бесконечности. После дискретной, ФС-топология является самой сильной из естественных топологий на $\mathscr{J}(\mathbf{k})$. Эта топология имеет следующий очевидный недостаток. Если основное кольцо $\mathbf{k}$, в свою очередь, снабжено некоторой (естественной) топологией, как, например, $\mathbf{k}=\mathbb{Q}, \mathbb{R}, \mathbb{C}, \mathbb{Z}_{(p)}, \mathbb{Q}(p), \ldots$, то ФС-топология совершенно нечувствительна к последней и всегда "воспринимает" k как дискретное кольцо. Чтобы устранить этот недостаток, введем на $\mathscr{J}(\mathbf{k})$ топологию, более тонкую, чем ФС-топология, которая была бы чувствительна к исходной топологии $\mathbf{k}$.

2.4. Обратные пределы топологических групп; топология на $\mathscr{J}(\mathbf{k})$. Рассмотрим (бесконечную) обратную последовательность множеств и отображений

$$
X_{1} \stackrel{p_{1}^{2}}{\longleftarrow} X_{2} \stackrel{p_{2}^{3}}{\longleftarrow} X_{3} \stackrel{p_{3}^{4}}{\longleftarrow} \cdots \stackrel{p_{n-1}^{n}}{\longleftarrow} X_{n} \stackrel{p_{n}^{n+1}}{\longleftarrow} \cdots .
$$


Если все множества $\left\{X_{n}\right\}_{n=1}^{\infty}$ наделены какой-то алгебраической структурой (группы, модули, кольца, ...), то отображения $\left\{p_{n}^{n+1}\right\}_{n=2}^{\infty}$ предполагаются гомоморфизмами; если $\left\{X_{n}\right\}_{n=1}^{\infty}-$ топологические пространства, то $\left\{p_{n}^{n+1}\right\}_{n=1}^{\infty}$ автоматически предполагаются непрерывными отображениями. Пусть

$$
X=\left\{\left(x_{1}, x_{2}, \ldots, x_{n}, \ldots\right) \mid x_{n} \in X_{n}, x_{n}=p_{n}^{n+1}\left(x_{n+1}\right), n=1,2, \ldots\right\}=\underset{n}{\lim } X_{n}
$$

- обратный предел последовательности (8), наделенный соответствующей структурой (группы, модуля, кольца, ... и/или топологического пространства) в зависимости от исходной структуры на $\left\{X_{n}\right\}_{n=1}^{\infty}$. Очевидным образом определены проекции на конечные факторы

$$
p_{n}: X \rightarrow X_{n}, \quad n=1,2, \ldots
$$

Заметим, что в случае, когда $\left\{X_{n}\right\}_{n=1}^{\infty}-$ топологические пространства, топология, возникающая на обратном пределе (9), будет наименьшей топологией, в которой все отображения (10) непрерывны.

Пример 2.6. Пусть $q$ - натуральное число и $X_{n}=S^{1}, n \in \mathbb{N}$, где окружность понимается как множество комплексных чисел, по модулю равных единице. Положим $p_{n}^{n+1}(z)=z^{q}$ для всех $n \in \mathbb{N}$. Рассматривая окружность как одномерную топологическую группу, мы получим в качестве обратного предела $S(q)=\lim _{n} X_{n}$ одномерную компактную топологическую группу, называемую $q$-адическим соленоидом. Рекомендуем читателю проверить, что в $S(q)$ уже нет элементов конечного порядка и что существует мономорфизм $\phi: \mathbb{R} \rightarrow S(q)$ топологических групп.

Пример 2.7. Все члены $X_{n}$ в $(8)$ - конечные группы. В этом случае соответствующий обратный предел $X$ называется проконечной группой. Конечные множества наделяются дискретной топологией, и, таким образом, проконечная группа $X$ естественно превращается в топологическую группу.

Проконечная группа $X$ компактна и имеет размерность 0 . Если все группы в (8) нетривиальны, а все проекции $p_{n}^{n+1}$ - эпиморфизмы, причем бесконечное число этих проекций имеет нетривиальное ядро, то $X$ не имеет изолированных точек и, следовательно, является канторовым множеством. С общей теорией проконечных групп можно познакомиться в [96] или [110].

ПримеР 2.8. Пусть $q$ - простое число, предположим, что все $X_{n}$ в (8) являются $q$-группами. В этом случае обратный предел $X$ последовательности (8) называется nро-q-группой. Группа целых $q$-адических чисел $\mathbb{Z}_{(q)}$ возникает как важный частный случай этой общей конструкции.

Пусть $X_{n}=\left\{z \in \mathbb{C} \mid z^{q^{n}}=1\right\}$ - группа корней степени $q^{n}$ из единицы. Положим $p_{n}^{n+1}(z)=z^{q}, z \in X_{n}$, и обозначим $R(q)$ соответствующий обратный предел; если $q$ - простое число, то $R(q) \simeq \mathbb{Z}_{(q)}$.

Несложно показать, что точной является следующая последовательность топологических групп:

$$
1 \longrightarrow R(q) \longrightarrow S(q) \stackrel{p_{1}}{\longrightarrow} S^{1} \longrightarrow 1
$$


Рекомендуем читателю разобраться, что происходит с подгруппой $\mathbb{R} \subset S(q)$ (см. пример 2.6) в этой точной последовательности.

Пример 2.9. Для группы $\mathscr{J}(\mathbf{k})$ формальных степенных рядов имеем следующее разложение:

$$
\mathscr{J}(\mathbf{k})=\lim _{n} \mathscr{J}_{n}(\mathbf{k})
$$

Если $\mathbf{k}$ - топологическое кольцо, то с помощью канонических координат группа $\mathscr{J}_{n}(\mathbf{k})$, как множество, отождествляется с

$$
\mathscr{J}_{n}(\mathbf{k}) \simeq \underbrace{\mathbf{k} \times \mathbf{k} \times \cdots \times \mathbf{k}}_{n \text { раз }} ;
$$

и становится топологическим пространством. Легко убедиться, что формулы $\left(4_{1}\right)-\left(4_{n}\right)$ непрерывны в топологии прямого произведения (12). Таким образом, группа $\mathscr{J}_{n}(\mathbf{k})$ становится топологической группой для любого топологического кольца $\mathbf{k}$, а проекции

$$
p_{n}^{n+1}: \mathscr{J}_{n+1}(\mathbf{k}) \rightarrow \mathscr{J}_{n}(\mathbf{k})
$$

становятся непрерывными отображениями. В случае топологического кольца $\mathbf{k}$ наделим группу $\mathscr{J}(\mathbf{k})$ топологией обратного предела (11) и будем далее называть такую топологию на $\mathscr{J}(\mathbf{k})$ естественной топологией.

ЗАмЕчАнИЕ 2.10. Если $\mathbf{k}$ дискретно, то естественная топология на $\mathscr{J}(\mathbf{k})$ совпадает с ФС-топологией. Если изначально на $\mathbf{k}$ не было задано никакой топологии, то мы считаем, что оно дискретно и снабжаем $\mathscr{J}(\mathbf{k})$ естественной топологией.

2.5. $\mathscr{J}(\mathbf{k})$ как группа автоморфизмов кольца $\mathbf{k}[[x]]$. Рассмотрим кольцо $\mathbf{k}[[x]]$ формальных степенных рядов с коэффициентами в кольце $\mathbf{k}$, произвольный элемент этого кольца имеет вид

$$
r(x)=\rho_{0}+\rho_{1} x+\rho_{2} x^{2}+\cdots, \quad \rho_{n} \in \mathbf{k}, \quad n=0,1, \ldots .
$$

Подмножества

$$
M_{n}=\left\{\rho_{n} x^{n}+\rho_{n+1} x^{n+1}+\cdots\right\}
$$

являются идеалами в $\mathbf{k}[[x]]$. Если $\mathbf{k}-$ поле, то $M_{1}$ - единственный максимальный идеал, $\mathbf{k}[[x]] / M_{1} \simeq \mathbf{k}$, т. е. $\mathbf{k}[[x]]$ - локальное кольцо.

Как описать группу автоморфизмов кольца $\mathbf{k}[[x]]$, которые постоянны на основном кольце $\mathbf{k}$, а также тривиально действуют на $M_{1}$ modulo $M_{2}$ ?

Если $A$ - некоторый элемент этой группы автоморфизмов, то достаточно знать, как $A$ действует на $x$. Предположим, что

$$
A(x)=x+r_{2}(x)=x+\rho_{1} x^{2}+\rho_{2} x^{3}+\cdots,
$$

тогда для всех $r(x) \in \mathbf{k}[[x]]$ имеем

$$
A: r(x) \mapsto r\left(x+r_{2}(x)\right) .
$$

Из (13) мы получаем, что $A$ действует на $\mathbf{k}[[x]]$ как элемент $f(x)=x+r_{2}(x)$ группы $\mathscr{J}(\mathbf{k})$. Обратно, любой $f(x) \in \mathscr{J}(\mathbf{k})$ действует на $\mathbf{k}[[x]]$ по формуле (13). Таким образом, справедлива следующая теорема. 
Tеорема 2.11. Группа $\mathscr{J}(\mathbf{k})$ изоморфна группе автоморфизмов кольца $\mathbf{k}[[x]]$, постоянных на $\mathbf{k}$ и сохраняющих $M_{1}$ modulo $M_{2}$.

ЗАмечание 2.12. Рассмотрим нильпотентное кольцо $M_{1} / M_{n+1}$. Тогда группа автоморфизмов $M_{1} / M_{n+1}$, тождественных на $\mathbf{k}$, совпадает с $\mathscr{J}_{n}(\mathbf{k})$.

2.6. Однопараметрические подгруппы. Скажем, что $G \subset \mathscr{J}(\mathbf{k})$ - однопараметрическая подгруппа, если она изоморфна аддитивной группе $\mathbf{k}$. По крайней мере одна однопараметрическая подгруппа существует для любого кольца $\mathbf{k}$.

ПримеР 2.13. Рассмотрим отображение групп $\mu: \mathbf{k} \rightarrow \mathscr{J}(\mathbf{k})$, определенное формулой

$$
\mu(\alpha)=x\left(1+\sum_{n=1}^{\infty} \alpha^{n} x^{n}\right) .
$$

Используя формулу геометрической прогрессии, несложно показать, что $\mu-$ мономорфизм группы $(\mathbf{k},+)$ в $\mathscr{J}(\mathbf{k})$.

Если $\mathbf{k}=\mathbb{Z}$, то любой элемент $f \in \mathscr{J}(\mathbb{Z})$ порождает однопараметрическую подгруппу $\left\{f^{n}\right\}_{n \in \mathbb{Z}}$. В дальнейшем мы увидим, что существуют другие, гораздо менее очевидные однопараметрические подгруппы.

Скажем, что $\mathbf{k}$ - кольцо характеристики 0 , если для любого $\alpha \in \mathbf{k}$ из равенства $n \alpha=0, n \in \mathbb{N}$, вытекает, что $\alpha=0$. Пусть $q$ - простое число; скажем, что $\mathbf{k}$ имеет характеристику $q$, если $q \alpha=0$ для любого $\alpha \in \mathbf{k}$.

Если $\mathbf{k}$ - область целостности нулевой характеристики, то об однопараметрических подгруппах в $\mathscr{J}(\mathbf{k})$ можно сказать достаточно много. Любая однопараметрическая подгруппа состоит из взаимно коммутирующих элементов. Для областей целостности нулевой характеристики верно и обратное: любые два коммутирующих элемента группы $\mathscr{J}(\mathbf{k})$ лежат в одной однопараметрической подгруппе. Прояснить ситуацию позволяет следующее полезное техническое утверждение.

ЛЕмма 2.14. Пусть $\mathbf{k}$ - область иелостности нулевой характеристики и

$$
f(x)=x\left(1+\alpha_{r} x^{r}+\cdots\right), \quad \alpha_{r} \neq 0,
$$

- некоторый элемент группы $\mathscr{J}(\mathbf{k})$. Предположим, что элемент $g(x)=x(1+$ $\left.\beta_{s} x^{s}+\cdots\right)$, где $\beta_{s} \neq 0$, коммутирует $c f$. Тогда $g$ однозначно определяется своим коэфбициентом $\beta_{s}$.

ДокАЗАтЕльство. Если $f$ и $g$ коммутируют, то $s=r$ согласно лемме $2.3, \mathrm{~b})$. Предположим, что существует еще один элемент $g^{\prime}(x)=x\left(1+\beta_{r} x^{r}+\cdots\right)$, коммутирующий с $f$. Из той же самой леммы 2.3 следует, что $g^{\prime} \circ g^{-1}=x(1+$ $\left.\mathscr{O}\left(x^{r+1}\right)\right)$ и этот элемент также должен коммутировать с $f$. Снова применяя лемму $2.3, \mathrm{~b})$, мы заключаем, что $\mathscr{O}\left(x^{r+1}\right)=0$.

ПРЕДЛОЖЕНИЕ 2.15. а) Пусть $\mathbf{k}$ - кольцо нулевой характеристики, тогда любой отличный от единицы элемент группы $\mathscr{J}(\mathbf{k})$ имеет бесконечный порядок. 
b) Два элемента $f$ u $g$ из $\mathscr{J}(\mathbb{Z})$ коммутируют $(f \circ g=g \circ f)$ тогда и только тогда, когда существует (единственньй) третий элемент $h \in \mathscr{J}(\mathbb{Z})$ такой, чmo

$$
f=h^{n} \quad u \quad g=h^{k} .
$$

с) В более общем виде: если $\mathbf{k}$ - целостное кольцо характеристики нуль, то два элемента $f$ и $g$ из $\mathscr{J}(\mathbf{k})$ коммутируют тогда и только тогда, когда они принадлежат одной однопараметрической подгруппе.

Заметим, что утверждение b) этого предложения не обобщается на евклидовы кольца (нулевой характеристики). Оно неверно, например, если $\mathbf{k}=$ $\mathbb{Z}[i]$ - кольцо целых гауссовых чисел. В действительности, часть с) предложения 2.15 - это наиболее удобный способ описания коммутирующих элементов в $\mathscr{J}(\mathbf{k})$ для целостных колец нулевой характеристики (см. п. 4.4).

ДоказАтельство. а) Рассмотрим элемент $f \in \mathscr{J}(\mathbf{k})$ вида (14). Тогда из леммы 2.3, с) вытекает, что $f^{n}(x)=x\left(1+n \alpha_{r} x^{r}+\cdots\right)$. Равенство $f^{n}(x)=x$ влечет $n \alpha_{r}=0$, откуда $\alpha_{r}=0$, что противоречит выбору $f$ в (14).

b) Предположим, что $g(x)=x\left(1+\beta_{s} x^{s}+\cdots\right)$, где $\beta_{s} \neq 0$, коммутирует с $f$ вида (14). Из леммы 2.3, b) вытекает, что $s=r$. Для упрощения обозначений далее полагаем $\alpha_{r}=\alpha$ и $\beta_{r}=\beta$, и пусть $\gamma=(\alpha, \beta)-$ наибольший общий делитель этих чисел:

$$
\gamma=u \alpha+v \beta, \quad \alpha=\gamma \alpha^{\prime}, \quad \beta=\gamma \beta^{\prime}
$$

Положим $h=f^{u} \circ g^{v}=x\left(1+\gamma x^{r}+\cdots\right)$. Мы видим, что $f$ коммутирует с $h^{\alpha^{\prime}}=x\left(1+\alpha x^{r}+\cdots\right)$ и, согласно лемме 2.14 , получаем $f=h^{\alpha^{\prime}}$. Второе равенство $g=h^{\beta^{\prime}}$ получается аналогичным образом.

Для доказательства с) следует использовать отображение ехр (см. п. 5.4) для группы $\mathscr{J}(\widehat{\mathbf{k}})$, где $\widehat{\mathbf{k}}-$ поле частных кольца $\mathbf{k}$, и лемму 2.14. Другая возможность состоит в рассмотрении обратной последовательности (11) и отыскании однопараметрических подгрупп в каждом члене $\mathscr{J}_{n}(\mathbf{k})$, используя мальцевскую теорию нильпотентных групп без кручения. Детали мы оставляем читателю.

ЗАмЕчАНИЕ 2.16. С помощью экспоненциального отображения (п. 5.4) несложно показать, что, в случае поля нулевой характеристики $\mathbf{k}$, через любой неединичный элемент $f \in \mathscr{J}(\mathbf{k})$ проходит (единственная) однопараметрическая подгруппа в $\mathscr{J}(\mathbf{k})$. Ситуация радикально меняется в положительной характеристике. Так, например, если $\mathbf{k}-$ конечное кольцо характеристики $p$ без делителей нуля и $d \equiv 0 \bmod p$, то элемент вида $f(x)=x\left(1+\alpha x^{d}+\mathscr{O}\left(x^{d+1}\right)\right)$ будет иметь бесконечный порядок и через него не может проходить никакая однопараметрическая подгруппа $\mathscr{J}(\mathbf{k})$.

2.7. Некоторые топологические свойства группы $\mathscr{J}(\mathbf{k})$. Топологические свойства группы $\mathscr{J}(\mathbf{k})$ находятся в прямой зависимости от топологических свойств базисного кольца $\mathbf{k}$. Так, если кольцо $\mathbf{k}$ компактно, например конечно, то $\mathscr{J}(\mathbf{k})$ - компактная топологическая группа. Если $\mathbf{k}$ дискретно и бесконечно, то $\mathscr{J}(\mathbf{k})$ уже не локально компактна и, как мы увидим, даже не 
вкладывается ни в какую локально компактную группу. Прежде чем сформулировать соответствующие утверждения, уточним терминологию.

Будем говорить, что топологическая группа $G$ уплотняется в топологическую группу $H$, если существует непрерывный мономорфизм $\phi: G \rightarrow H$. Если такой мономорфизм $\phi$ является еще и гомеоморфизмом на свой образ, то будем говорить, что группа $G$ вкладывается в топологическую группу $H$.

ПримеР 2.17. Рассмотрим аддитивные группы $\mathbb{R}$ и $T^{2}=S^{1} \times S^{1}$, снабженные обычной топологией. Отображение $\phi(t)=\left(\mathbf{e}^{i t}, \mathbf{e}^{i \sqrt{2} t}\right)$ задает уплотнение $\mathbb{R}$ в $T^{2}$, но это отображение не будет вложением топологических групп.

ПримеР 2.18. Если некоторое топологическое кольцо $\mathbf{k}$ уплотняется в компактное кольцо $\mathbf{k}^{\prime}$, то группа $\mathscr{J}(\mathbf{k})$, очевидно, уплотняется в группу $\mathscr{J}\left(\mathbf{k}^{\prime}\right)$, которая компактна. С этой точки зрения группа $\mathscr{J}(\mathbb{Z})$, не являясь ни компактной, ни локально компактной, уплотняется в компактную группу. Наиболее простое и естественное уплотнение $\mathscr{J}(\mathbb{Z})$ в компактную группу можно получить, снабдив $\mathbb{Z} p$-адической топологией и вложив $\mathbb{Z} \subset \mathbb{Z}(p)$ в кольцо целых $p$-адических чисел для некоторого простого $p$. В силу компактности кольца $\mathbb{Z}_{(p)}$ в $p$-адической топологии, группа $\mathscr{J}\left(\mathbb{Z}_{(p)}\right)$ также компактна, причем $\mathscr{J}(\mathbb{Z})$ будет в ней всюду плотной подгруппой.

Предыдущее уплотнение основано на уплотнении основного кольца. Интересно, что $\mathscr{J}(\mathbb{Z})$ уплотняется также в группу $\mathscr{J}\left(\mathbb{Z}_{p}\right)$. Такие уплотнения уже будут неявными и основаны на аппроксимируемости $\mathscr{J}(\mathbb{Z})$ конечными p-группами и теореме вложения 4.10.

Ситуация меняется, если мы попытаемся вложить $\mathscr{J}(\mathbb{Z})$ в какую-нибудь локально компактную группу, нижеследующие результаты доказаны в [3].

Теорема 2.19. Если аддитивная группа топологического кольиа $\mathbf{k}$ содержит бесконечную дискретную подгруппу, то группа $\mathscr{J}(\mathbf{k})$ не вкладывается в локально компактную группу.

СлеДСтвие 2.20. Группа $\mathscr{J}(\mathbb{Z})$ не вкладывается ни в какую локально компактную группу.

Оказывается, что чисто алгебраические свойства базисного кольца $\mathbf{k}$ также влияют на возможную вложимость группы $\mathscr{J}(\mathbf{k})$ в компактные и локально компактные группы.

Теорема 2.21. Пусть $G$ - локально компактная группа и $\phi: \mathscr{J}(\mathbb{Q}) \rightarrow G$ (непрерывный) гомоморфизм. Тогда найдется натуральное $m$ такое, что $\mathscr{J}^{m}(\mathbb{Q}) \subset \operatorname{ker} \phi$. Если при этом группа $G$ компактна, то $\mathscr{J}^{3}(\mathbb{Q}) \subset \operatorname{ker} \phi$.

СлеДСтвиЕ 2.22. Пусть $\mathbf{k}$ - топологическое кольцо, содержащее поле рациональных чисел. Тогда группа $\mathscr{J}(\mathbf{k})$ не уплотняется ни в какую локально компактную группу.

Здесь мы не делаем никаких дополнительных предположений о топологии кольца $\mathbf{k}$, никакие топологические свойства включения $\mathbb{Q} \subset \mathbf{k}$ также не предполагаются. 
Важным свойством группы $\mathscr{J}(\mathbb{Q})$ и всех связанных с ней групп $\mathscr{J}^{m}(\mathbb{Q})$, $m>1$, является их алгебраическая полнота, или бесконечная делимость. Это чисто алгебраическое свойство, в конечном итоге, и определяет невозможность вложения в локально компактные группы, детали см. в [3].

2.8. Группа автоморфизмов группы $\mathscr{J}(\mathbf{k})$ и группа $\mathscr{A}(\mathbf{k})$. Под автоморфизмами группы $\mathscr{J}(\mathbf{k})$ мы всегда понимаем непрерывные автоморфизмы. Каждый (непрерывный) автоморфизм кольца $\mathbf{k}, \varphi \in \operatorname{Aut}(\mathbf{k})$, очевидно действует на $\mathscr{J}(\mathbf{k})$ по правилу

$$
\varphi\left(x\left(1+\sum_{i=1}^{\infty} \alpha_{i} x^{i}\right)\right)=x\left(1+\sum_{i=1}^{\infty} \varphi\left(\alpha_{i}\right) x^{i}\right),
$$

задавая вложение $\operatorname{Aut}(\mathbf{k}) \subset \operatorname{Aut}(\mathscr{J}(\mathbf{k}))$. Другой очевидный набор автоморфизмов группы $\mathscr{J}(\mathbf{k})$ составляют ее внутренние автоморфизмы.

$\mathrm{K}$ перечисленным выше очевидным автоморфизмам группы $\mathscr{J}(\mathbf{k})$ следует добавить еще и гомотетии, подробно рассматриваемые в п. 2.3 в более общем контексте. Пусть $\mathbf{k}^{*}$ - мультипликативная группа обратимых элементов кольца $\mathbf{k}$. Для $t \in \mathbf{k}^{*}$ и $f(x)=x\left(1+\sum_{i=1}^{\infty} \alpha_{i} x^{i}\right) \in \mathscr{J}(\mathbf{k})$ положим

$$
\delta_{t}(f(x))=x\left(1+\sum_{i=1}^{\infty} \alpha_{i} t^{i} x^{i}\right)
$$

и назовем такое преобразование гомотетией с коэффициентом $t$. Записывая формально гомотетию как $\delta_{t}(f(x))=(1 / t) f(t x)$, легко видеть, что это автоморфизм группы $\mathscr{J}(\mathbf{k})$ для любого $t \in \mathbf{k}^{*}$.

В действительности гомотетии являются "почти" внутренними автоморфизмами группы $\mathscr{J}(\mathbf{k})$. Рассмотрим полную группу формальных диффеоморфизмов k-прямой, состоящую из всех формальных рядов вида

$$
\mathscr{A}(\mathbf{k})=\left\{f(x)=\sum_{i=0}^{\infty} \alpha_{i} x^{i+1}, \alpha_{0} \in \mathbf{k}^{*} ; \alpha_{i} \in \mathbf{k}, i>0\right\}
$$

с той же операцией подстановки ряда в ряд. Коэффициент $\alpha_{0}$ часто называют мулътипликатором элемента $f(x)$. Очевидно, что $\mathscr{A}(\mathbf{k})$ - это группа и $\mathscr{J}(\mathbf{k})-$ нормальная подгруппа в ней.

ЗАмечАниЕ 2.23. Несложно показать, что $\mathscr{A}(\mathbf{k}) \simeq \mathbf{k}^{*} \ltimes \mathscr{J}(\mathbf{k})$. При этом структура полупрямого произведения задается на множестве $\mathbf{k}^{*} \times \mathscr{J}(\mathbf{k})$ операцией

$$
(\alpha, f(x)) \circ(\beta, g(x))=\left(\alpha \beta, \delta_{\beta}(f(x)) \circ g(x)\right),
$$

где $\alpha, \beta \in \mathbf{k}^{*}$ и $f(x), g(x) \in \mathscr{J}(\mathbf{k})$.

Теперь мы видим, что гомотетия $\delta_{t}$ является ограничением на $\mathscr{J}(\mathbf{k})$ внутреннего автоморфизма $\mathscr{A}(\mathbf{k})$, задаваемого элементом $t x, t \in \mathbf{k}^{*}$. Автоморфизм групп $\mathscr{A}(\mathbf{k})$ и $\mathscr{J}(\mathbf{k})$ назовем стандартным, если он является композицией внутреннего автоморфизма $\mathscr{A}(\mathbf{k})$ и автоморфизма, индуцированного кольцевым автоморфизмом. 
Первые исследования групп $\operatorname{Aut}(\mathscr{A}(\mathbf{k}))$ и $\operatorname{Aut}(\mathscr{J}(\mathbf{k}))$ были проведены Макенхоуптом более 50 лет назад [82]. Все известные к настоящему времени результаты об этих группах доказаны в предположении, что $\mathbf{k}$ - поле, причем характеристики, отличной от 2 . То, что $\mathbf{k}$ является полем, - вполне естественное предположение, пример 2.25 ниже показывает, что вряд ли стоит ожидать разумного описания групп автоморфизмов $\operatorname{Aut}(\mathscr{A}(\mathbf{k}))$ и $\operatorname{Aut}(\mathscr{J}(\mathbf{k}))$ для колец общего вида.

ТЕОРема 2.24. Пусть $\mathbf{k}$ - поле характеристики $p \neq 2$, причем если $\mathbf{k} к о-$ нечно, то $p \geqslant 5$. Любой автоморфизм группь $\mathscr{A}(\mathbf{k})$, так же как и любой автоморфизм $\mathscr{J}(\mathbf{k})$, является стандартным. Более того, имеет место изоморфизм

$$
\operatorname{Aut}(\mathscr{A}(\mathbf{k})) \simeq \operatorname{Aut}(\mathscr{J}(\mathbf{k})) \simeq \operatorname{Aut}(\mathbf{k}) \ltimes \mathscr{A}(\mathbf{k}) .
$$

Этот результат был первоначально доказан Макенхоуптом [82] для полей нулевой характеристики и бесконечных полей конечной характеристики, отличной от 2. Случай конечных полей характеристики $p \geqslant 5$ был доказан Клопшем [65]. Вопрос об автоморфизмах групп $\mathscr{A}(\mathbf{k})$ и $\mathscr{J}(\mathbf{k})$ в случае поля $\mathbf{k}$ характеристики 2 остается полностью неизученным.

Следующий пример показывает, что для достаточно простых кольц $\mathbf{k}$ ситуация может быть совсем не так прозрачна, как в теореме 2.24. В целях упрощения мы рассматриваем здесь кольцо без единицы; как уже отмечалось выше, определение группы $\mathscr{J}(\mathbf{k})$ над такими кольцами не теряет смысл.

ПримеР 2.25. Рассмотрим кольцо из двух элементов $\mathbf{k}=\{0, a \mid a+a=$ $a a=0\}$. Несложно проверить, что группа $\mathscr{J}(\mathbf{k})$ абелева, более того, $\mathscr{J}(\mathbf{k}) \simeq$ $\mathbb{Z}_{2}^{\mathbb{N}}$. Мы видим, что $\mathscr{J}(\mathbf{k})$ не имеет никаких стандартных автоморфизмов, тем не менее группа $\operatorname{Aut}(\mathscr{J}(\mathbf{k}))$ чрезвычайно велика и структура ее элементов совершенно отлична от структуры стандартных автоморфизмов.

\section{3. Сжимаемость}

3.1. Сжимаемые топологические группы. В этом разделе мы рассматриваем свойство типа фрактальности, присущее группе $\mathscr{J}(\mathbf{k})$ для очень многих базисных колец $\mathbf{k}$. Систематическое изучение этого свойства было начато в [3], где можно найти и доказательства нижеследующих утверждений.

ОПРеДЕЛЕНИЕ 3.1. Пусть $G$ - топологическая группа. Скажем, что $G$ сжимаема, если для любой окрестности единицы $e \in U \subset G$ существует вложение

$$
\phi_{U}: G \rightarrow G,
$$

такое, что $\operatorname{Im} \phi_{U} \subset U$. Вложение $\phi_{U}$ называется сжатием, соответствующим окрестности $U$.

ПримеР 3.2. Пусть $H$ - топологическая группа, положим $G=H^{\mathbb{N}}$. Легко видеть, что $G$ - сжимаемая топологическая группа. Правые сдвиги

$$
\phi_{n}\left(g_{1}, g_{2}, \ldots\right)=\left(e, e, \ldots, e, g_{1}, g_{2}, \ldots\right), \quad g_{1} \text { на }(n+1) \text {-м месте }
$$

дают необходимую последовательность сжатий. 
ПримеР 3.3. Рассмотрим группу $G=$ Homeо $+_{+}[0,1]$ гомеоморфизмов отрезка $[0,1]$, сохраняющих его концы. Мы рассматриваем $G$ в естественной топологии группы гомеоморфизмов метрического пространства. Пусть $f(x) \in G$, положим

$$
\phi_{n}(f(x))=\frac{1}{n} f(n x), \quad 0 \leqslant x \leqslant \frac{1}{n} ; \quad \phi_{n}(f(x))=x, \quad \frac{1}{n} \leqslant x \leqslant 1 .
$$

Легко видеть, что $\phi_{n}$ является вложением при любом $n \in \mathbb{N}$ и, кроме того, для любой окрестности $U$ единицы $e \in G$ найдется натуральное $n$ такое, что $\operatorname{Im} \phi_{n} \subset U$. Это означает, что вложения $\left\{\phi_{n}\right\}_{n=1}^{\infty}$ образуют систему сжатий группы $G$.

Заметим, что группа Томпсона $F$, состоящая из 2-адических гомеоморфизмов отрезка $[0,1]$ (см. [26]), является подгруппой $G$ и это включение согласовано со сжатиями $\left\{\phi_{2^{n}}\right\}_{n=1}^{\infty}$. Иначе говоря, отображения $\left\{\phi_{2^{n}}\right\}_{n=1}^{\infty}$ образуют систему сжатий группы $F$, если ее снабдить топологией, индуцированной естественным вложением $F \hookrightarrow G$. Эта топология совершенно отлична от дискретной топологии, обычно рассматриваемой на $F$.

Ввиду отсутствия малых подгрупп, сжимаемость никогда не возникает в группах Ли. Пример 3.2 показывает, что сжимаемые топологические группы могут иметь размерность 0 или бесконечность. Следующий результат [3] утверждает, что для компактных групп это действительно единственно возможные размерности.

ТЕорема 3.4. Любая компактная сжсимаемая группа имеет размерность 0 или бесконечность.

При изучении сжимаемости группы $\mathscr{J}(\mathbf{k})$ нам будут полезны несколько фактов классического анализа.

3.2. Одна теорема Эйзенштейна об алгебраических функциях. Рассмотрим функцию $(1+z)^{1 / s}$, где $s$ - натуральное число, большее единицы. Разлагая ее в биномиальный ряд, мы получим ряд Тейлора, коэффициенты которого рациональные, но не целые. Тем не менее справедливо следующее утверждение, доказательство которого может служить неплохим упражнением.

ЛЕмма 3.5. Для любого натуралъного s функиия

$$
u(z)=\left(1+s^{2} z\right)^{1 / s}
$$

разлагается в окрестности нуля в ряд Тейлора с иелыми коэбфициентами, кратными $s$.

ЗАмечание 3.6. Допустим, что разложение $s$ на простые множители имеет вид $s=q_{1}^{m_{1}} q_{2}^{m_{2}} \cdots q_{l}^{m_{l}}$, и положим $\widehat{s}=q_{1}^{m_{1}+1} q_{2}^{m_{2}+1} \cdots q_{l}^{m_{l}+1}$. Можно показать [92], что функция

$$
u(z)=(1+\widehat{s} z)^{1 / s}
$$

разлагается в нуле в ряд с целыми коэффициентами. 
Лемма 3.5 и замечание 3.6 являются, в действительности, частными случаями общего результата, принадлежащего Эйзенштейну, в качестве ссылки cм. [92].

ТЕОРема 3.7. Пусть ряд $u(z)=\beta_{1} z+\beta_{2} z^{2}+\cdots$ имеет рациональные коэффициенты и представляет в нуле алгебраическую функиию. Тогда найдется натуральное число Т такое, что все коэффициенты ряда

$$
u(T z)=\beta_{1} T z+\beta_{2} T^{2} z^{2}+\cdots
$$

являются целыми числами.

Наименьшее число T, удовлетворяющее теореме 3.7, назовем индексом Эйзенштейна. В общем случае его достаточно трудно вычислить. Замечание 3.6 показывает, что ответ нетривиален даже в наипростейшем случае, которому посвящена лемма 3.5. Число $s^{2}$, как правило, превосходит индекс Эйзенштейна $\widehat{s}$, но оно гораздо проще и полностью удовлетворяет нашим техническим нуждам.

3.3. Сжимаемость группы $\mathscr{J}(\mathbf{k})$. Группа $\mathscr{J}(\mathbf{k})$ имеет несколько замечательных эндоморфизмов. Для любого элемента $f(x) \in \mathscr{J}(\mathbf{k})$ и для любого $t \in \mathbf{k}$ рассмотрим гомотетию

$$
\delta_{t}(f)=x\left(1+t \alpha_{1} x+t^{2} \alpha_{2} x^{2}+\cdots\right)=\frac{1}{t} f(t x) .
$$

Здесь левое равенство корректно определяет $\delta_{t}(f)$, а правое равенство, понимаемое формально, показывает, что $\delta_{t}(f)$ является эндоморфизмом группы $\mathscr{J}(\mathbf{k})$. Если $t$ не является делителем нуля в $\mathbf{k}$, то $\delta_{t}$ будет алгебраическим мономорфизмом. Если к тому же кольцо $\mathbf{k}$ дискретно, то $\delta_{t}$ будет вложением группы $\mathscr{J}(\mathbf{k})$ в себя. Если $\mathbf{k}-$ топологическое поле и $t \in \mathbf{k}^{*}$, то, как мы видели выше, $\delta_{t}$ будет автоморфизмом группы $\mathscr{J}(\mathbf{k})$, причем обратным автоморфизмом является $\delta_{1 / t}$.

Для любых $t, r \in \mathbf{k}$ непосредственно из (15) заключаем, что $\delta_{t}$ о $\delta_{r}=\delta_{t r}$. Иначе говоря, отображение $\delta_{t}$ осуществляет вложение мультипликативной группы $\mathbf{k}^{*}$ обратимых элементов кольца $\mathbf{k}$ в группу автоморфизмов $\operatorname{Aut}(\mathscr{J}(\mathbf{k}))$.

Чтобы определить еще один эндоморфизм группы $\mathscr{J}(\mathbf{k})$, перепишем произвольный элемент $f(x)=x\left(1+\alpha_{1} x+\alpha_{2} x^{2}+\cdots\right)$ этой группы в следующей форме:

$$
f(x)=x(1+x h(x)),
$$

где $h(x)=\alpha_{1}+\alpha_{2} x+\cdots$. Далее для натурального $s$ положим

$$
\Theta_{s}(f)=x\left(1+s^{2} x^{s} h\left(s^{2} x^{s}\right)\right)^{1 / s},
$$

где правая часть предполагает разложение в биномиальный ряд с дальнейшим возведением $h\left(s^{2} x^{s}\right)$ в последовательные степени и последующим приведением подобных членов. То, что коэффициенты полученного ряда принадлежат кольцу $\mathbf{k}$, обеспечивается леммой 3.5. 
Для изучения отображения (16) введем следующее подмножество $\mathscr{J}(\mathbf{k})$ :

$$
\mathscr{J}_{(s)}(\mathbf{k})=\left\{x\left(1+\alpha_{1} x^{s}+\alpha_{2} x^{2 s}+\cdots\right), \alpha_{n} \in \mathbf{k}\right\} \subset \mathscr{J}(\mathbf{k}) .
$$

Очевидно, что $\mathscr{J}(s)(\mathbf{k})$ - замкнутая подгруппа в $\mathscr{J}(\mathbf{k})$, кроме того, для любого натурального $s$

$$
\mathscr{J}_{(s)}(\mathbf{k}) \subset \mathscr{J}^{s}(\mathbf{k}) .
$$

ПреДЛОЖЕНИЕ 3.8. Для любого натуралъного s отображение $\Theta_{s}$ корректно определено и является гомоморфизмом группь $\mathscr{J}(\mathbf{k})$ в группу $\mathscr{J}_{(s)}(\mathbf{k})$.

Если элемент $s=s \mathbf{1}, \mathbf{1} \in \mathbf{k}$, не является делителем нуля в $\mathbf{k}$, то $\Theta_{s}-$ мономорфизм; если, кроме того, $\mathbf{k}$ дискретно (либо является одним из колеи, $\mathbb{Q}$, $\mathbb{R}, \mathbb{C}, \mathbb{Z}_{(p)}, \mathbb{Q}_{(p)}$, снабженных обычной топологией), то $\Theta_{s}$ - вложение.

Если s обратим в $\mathbf{k}$, то $\Theta_{s}-$ изоморбизм группь $\mathscr{J}(\mathbf{k})$ на группу $\mathscr{J}_{(s)}(\mathbf{k})$.

Доказательство этого утверждения можно найти в [3]. Отметим лишь, что имеет место полезное формальное представление $\Theta_{s}$ в виде

$$
\Theta_{s}=\theta_{s} \circ \delta_{s^{2}}
$$

где $\delta_{s^{2}}-$ гомотетия с параметром $s^{2}=s^{2} \mathbf{1}$ и

$$
\theta_{s}(f(x))=f\left(x^{s}\right)^{1 / s} .
$$

Гомоморфность отображения $\Theta_{s}$ вытекает из формулы (19).

ЗАмечАниЕ 3.9. а) Предположим, что натуральное число $s$ не делится на простое число $p$. Тогда для любого дискретного поля $\mathbf{k}$ характеристики $p$ формула $(20)$ корректно определена и задает изоморфизм $\mathscr{J}(\mathbf{k})$ на группу $\mathscr{J}_{(s)}(\mathbf{k})$.

b) Если основное кольцо $\mathbf{k}$ содержит поле рациональных чисел $\mathbb{Q}$, то конструкцию сжатия $\Theta_{s}$ можно упростить, исключив гомотетию $\delta_{s^{2}}$ из равенства (19) и просто положив $\Theta_{s}=\theta_{s}$. В этом случае формулы несколько упрощаются.

c) Сжатие $\theta_{s}$ на группе $\mathscr{J}(\mathbb{R})$ индуцирует эндоморфизм $\theta_{s}^{*}$ соответствующей бесконечномерной алгебры Ли (см. п. 4.3). В базисе $(31) \theta_{s}^{*}$ имеет простой вид

$$
\theta_{s}^{*}\left(e_{n}\right)=\frac{1}{s} e_{s n}, \quad n=1,2, \ldots
$$

Если интерпретировать элементы $W_{1}(1)$ как полиномиальные векторные поля на окружности, то отображение $\theta_{s}^{*}$ индуцируется $s$-листным накрытием окружности. Отображение $\theta_{s}^{*}$ носит универсальный характер - оно определено на всех алгебрах $W_{1}(k), k=0,1,2, \ldots$, где нумерация векторов базиса ведется по $n \geqslant k$, а также на полной алгебре Витта $W_{1}$, где $n$ пробегает все целые числа. Аналоги эндоморфизмов $\theta_{s}^{*}$ существуют и в конечной характеристике, когда $s$ взаимно просто с характеристикой.

d) Существуют кольца $\mathbf{k}$ такие, что $\Theta_{s}$ не будет мономорфизмом ни при каком $s \geqslant 2$. В этом случае предложенная конструкция сжатий неэффективна.

ОПРЕДЕЛЕНИЕ 3.10. Эндоморфизм $\Theta_{s}$, определенный в (16), будем называть сжатием с коэфбиииентом s. 
Если кольцо $\mathbf{k}$ таково, что предложение 3.8 имеет силу для бесконечной последовательности натуральных чисел $\left\{s_{i}\right\}_{i=1}^{\infty}$, то сжатия $\left\{\Theta_{s_{i}}\right\}_{i=1}^{\infty}$ являются вложениями, а включение (18), в свою очередь, гарантирует сжимаемость группы $\mathscr{J}(\mathbf{k})$. Таким образом, имеет место следующее утверждение.

СЛЕДСТВИЕ 3.11. Пусть кольщо $\mathbf{k}$ удовлетворяет одному из следующих условий:

а) $\mathbf{k}$ дискретно и для бесконечного числа натуральных чисел $s$ элемент $s \mathbf{1} \in \mathbf{k}$ не является делителем 0 в $\mathbf{k}$;

b) $\mathbf{k}$ - одно из колец, $\mathbb{Q}, \mathbb{R}, \mathbb{C}, \mathbb{Z}_{(p)}, \mathbb{Q}_{(p)}$, снабженных обычной топологией.

Тогда группа $\mathscr{J}(\mathbf{k})$ сжимаема.

Может создаться впечатление, что эффект сжимаемости группы $\mathscr{J}(\mathbf{k})$ присущ исключительно размерностям 0 и бесконечность. Это в действительности не так, закончим этот пункт следующим утверждением [3].

ПрЕДЛОЖЕНИЕ 3.12. Существует такое топологическое колъцо с единицей $\mathbf{k}$, что группа $\mathscr{J}(\mathbf{k})$ является одномерной сепарабельной метризуемой сжимаемой группой.

\section{4. Группа $\mathscr{J}\left(\mathbb{Z}_{p}\right)$}

4.1. Общие свойства. Если $p$ - простое число, то группа $\mathscr{J}\left(\mathbb{Z}_{p}\right)$ обладает рядом интересных свойств, которые мы рассмотрим в этом разделе. Для элемента $f \in \mathscr{J}\left(\mathbb{Z}_{p}\right)$ вида

$$
f(x)=x\left(1+\alpha_{n} x^{n}+\mathscr{O}\left(x^{n+1}\right)\right), \quad \alpha_{n} \neq 0,
$$

из леммы 2.3, с) вытекает, что $f^{p}(x)=x\left(1+\mathscr{O}\left(x^{n+1}\right)\right)$. Отсюда, индукцией по $k$, получаем, что для произвольного элемента $f \in \mathscr{J}\left(\mathbb{Z}_{p}\right)$ справедливо равенство

$$
f^{p^{k}}(x)=x\left(1+\mathscr{O}\left(x^{n+k}\right)\right) \in \mathscr{J}^{n+k-1}\left(\mathbb{Z}_{p}\right) .
$$

Следовательно, группы $\mathscr{J}_{n}\left(\mathbb{Z}_{p}\right), n=1,2, \ldots$, являются $p$-группами и, в силу представления $(11), \mathscr{J}\left(\mathbb{Z}_{p}\right)$ будет про-р-группой. Таким образом, мы видим, что $\mathscr{J}\left(\mathbb{Z}_{p}\right)$ компактная нульмерная (несчетная) группа, т. е. она гомеоморфна канторову совершенному множеству.

Легко видеть, что элемент

$$
f(x)=x\left(1+x+x^{2}+\cdots\right)=\frac{x}{1-x}
$$

имеет порядок $p$ и, следовательно, порождает в $\mathscr{J}\left(\mathbb{Z}_{p}\right)$ однопараметрическую подгруппу. Можно указать даже бесконечную последовательность однопараметрических подгрупп в $\mathscr{J}\left(\mathbb{Z}_{p}\right)$. Если $(s, p)=1$, то, применяя к $f(x)$ из $(21)$ отображение (20), получим бесконечную последовательность элементов

$$
f_{s}(x)=\theta_{s}(f(x))=\frac{x}{\left(1+x^{s}\right)^{1 / s}}, \quad s \in \mathbb{N}, \quad(s, p)=1 .
$$

Согласно замечанию 3.9 , а), элементы (22) порождают однопараметрические подгруппы в $\mathscr{J}\left(\mathbb{Z}_{p}\right)$. Эти подгруппы попарно различны даже с точностью до 
автоморфизма самой группы $\mathscr{J}\left(\mathbb{Z}_{p}\right)$. Можно показать (рекомендуем читателю это проделать в качестве хорошего упражнения), что ни через один элемент вида $f(x)=x\left(1+\alpha x^{m p}+\mathscr{O}\left(x^{m p+1}\right)\right), \alpha \neq 0$, не может проходить однопарамерическая подгруппа в $\mathscr{J}\left(\mathbb{Z}_{p}\right)$.

Оказывается, что с точностью до сопряженности все однопараметрические подгруппы в $\mathscr{J}\left(\mathbb{Z}_{p}\right)$ исчерпываются подгруппами, порожденными элементами (22). Это вытекает из следующего несколько более общего результата Клопша [65].

ТЕОРема 4.1. Пусть $\mathbf{k}=\mathbb{F}_{q}$ - конечное поле характеристики $p$, тогда подмножество элементов $\mathscr{J}(\mathbf{k})$ вида

$$
\left\{f_{s, \lambda}(x)=x\left(1+s \lambda x^{s}\right)^{-1 / s}, s \in \mathbb{N},(s, p)=1 ; \lambda \in \mathbf{k}^{*}\right\}
$$

взаимно однозначно представляет все классы сопряженных элементов порядка $p$.

4.2. $p$-группы $\mathscr{J}_{n}(\mathbf{k})$, для кольца характеристики $p$. Как было отмечено в предыдущем пункте, $\mathscr{J}_{n}\left(\mathbb{Z}_{p}\right)$ является $p$-группой для любого $n$. Вычисление экспоненты этой группы является важной и естественной задачей, которой посвящен настоящий пункт. Напомним, что для некоторой $p$-группы $G$ ее экспонентой $\exp (G)$ называется наименьшее натуральное $s$ такое, что $g^{p^{s}}=\mathbf{e}$ для любого $g \in G$.

Мы рассматриваем чуть более общую ситуацию, чем выше, и считаем, что $\mathbf{k}$ - произвольное кольцо простой характеристики $p$. Применяя систематически лемму $2.3, \mathrm{c})$, для любого элемента $f(x) \in \mathscr{J}(\mathbf{k})$ получаем, что $f^{p^{n}}(x) \in$ $\mathscr{J}^{n+1}(\mathbf{k})$. Можно заметить, что это включение слишком грубое и уже в основополагающей работе [59] Дженнингсом был получен существенно более сильный результат. Пусть $G$ - группа, условимся обозначать $G^{(k)}$ подгруппу, порожденную $k$-ми степенями всевозможных элементов из $G$.

Теорема 4.2. Если $\mathbf{k}$ - колъцо простой характеристики р, то для любого натурального $n$ имеет место включение $\left(\mathscr{J}^{n}(\mathbf{k})\right)^{(p)} \subseteq \mathscr{J}^{n p}(\mathbf{k})$.

Непосредственно из этого результата вытекает, что если $n+1 \leqslant p^{s}$, то порядок любого элемента группы $\mathscr{J}_{n}(\mathbf{k})$ не превосходит $p^{s}$. Иначе говоря, для любого кольца $\mathbf{k}$ характеристики $p$ экспонента $\mathscr{J}_{n}(\mathbf{k})$ не превосходит $s$. Например, можно показать (советуем читателю проделать это вычисление), что $\exp \left(\mathscr{J}_{3}\left(\mathbb{Z}_{2}\right)\right)=2$ и $\exp \left(\mathscr{J}_{3}\left(\mathbb{Z}_{p}\right)\right)=1$, если $p \neq 2$.

Точное вычисление $\exp \left(\mathscr{J}_{n}(\mathbf{k})\right)$ для кольца характеристики $p$ представляет собой достаточно тонкую задачу. Продолжая исследование Дженнингса, Йорк [115] существенно уточнил результат [59] и получил точную формулу для экспоненты. Сведем эти результаты в следующую теорему, детали см. в [115].

Теорема 4.3. Для кольца $\mathbf{k}$ с единицей характеристики $p \geqslant 3$

$$
\exp \left(\mathscr{J}_{n}(\mathbf{k})\right)=\left[\log _{p}(n(p-1))\right] .
$$

Если $\mathbf{k}$ - область иелостности характеристики 2 , отличная от $\mathbb{Z}_{2}$, то

$$
\exp \left(\mathscr{J}_{n}(\mathbf{k})\right)=\left[\log _{2} n\right] .
$$


Кроме того,

$$
\exp \left(\mathscr{J}_{n}\left(\mathbb{Z}_{2}\right)\right)= \begin{cases}{\left[\log _{2} n\right],} & \text { nри } n \geqslant 5 \text { u } n \neq 2^{m} \\ m-1, & \text { nрu } n=2^{m}>5 .\end{cases}
$$

Здесь, как обычно, [ [ ] означает целую часть числа. Мы видим, что наиболее сложное строение групп $\mathscr{J}_{n}(\mathbf{k})$ возникает в характеристике 2 . В дальнейшем мы еще не раз убедимся, что при изучении групп $\mathscr{J}(\mathbf{k})$ характеристика 2 оказывается более сложной, чем нечетная характеристика. Многие изученные в нечетной характеристике вопросы остаются открытыми в характеристике 2 и по сей день.

Для доказательства точности указанных в теореме значений экспоненты в [115] были предъявлены элементы максимального порядка в группах $\mathscr{J}_{n}(\mathbf{k})$. Оказывается, что в любой нечетной характеристике в качестве такого элемента можно взять $f(x)=x+x^{3}$. В случае $\mathbf{k}=\mathbb{Z}_{2}$ можно выбрать $f(x)=x+x^{2}$, а в остальных случаях характеристики 2 соответственно $f(x)=x+x^{2}+a x^{3}$, где коэффициент $а$ принадлежит $\mathbf{k}$ и отличен от 0 и 1.

4.3. Системы топологических образующих, определяющие соотношения. Будем говорить, что топологическая группа $G$ обладает системой образующих $g_{1}, g_{2}, \ldots, g_{n}$, если замыкание подгруппы $\left\langle g_{1}, g_{2}, \ldots, g_{n}\right\rangle$, порожденной элементами $g_{1}, g_{2}, \ldots, g_{n}$, совпадает с самой группой $G$ :

$$
\overline{\left\langle g_{1}, g_{2}, \ldots, g_{n}\right\rangle}=G \text {. }
$$

ПримеР 4.4. Рассмотрим $n$-мерный тор $T^{n}$. Являясь $n$-мерным многообразием, $T^{n}$, как топологическая группа, может быть порожден всего одним своим элементом.

Этот пример показывает серьезное различие между системами порождающих дискретной группы и топологической группы общего вида.

Теорема 4.5. а) Если $p>2$, то группа $\mathscr{J}\left(\mathbb{Z}_{p}\right)$ порождается не менее чем двумя образующими. Два элемента

$$
f_{1}(x)=x(1+x)+\mathscr{O}\left(x^{3}\right), \quad f_{2}(x)=x\left(1+x^{2}\right)+\mathscr{O}\left(x^{4}\right)
$$

порождают $\mathscr{J}\left(\mathbb{Z}_{p}\right)$ при любом выборе остаточных членов $\mathscr{O}\left(x^{i}\right), i=3,4$.

b) Группа $\mathscr{J}\left(\mathbb{Z}_{2}\right)$ порождается не менее чем тремя образующими. Следующие три элемента:

$$
f_{1}(x)=x(1+x)+\mathscr{O}\left(x^{3}\right), \quad f_{2}(x)=x\left(1+x^{2}\right)+\mathscr{O}\left(x^{4}\right), \quad f_{6}(x)=x\left(1+x^{6}\right)+\mathscr{O}\left(x^{8}\right)
$$

порождают $\mathscr{J}\left(\mathbb{Z}_{2}\right)$ при любом выборе остаточных членов $\mathscr{O}\left(x^{i}\right), i=3,4,8$.

Для доказательства минимальности числа образующих группы $\mathscr{J}\left(\mathbb{Z}_{p}\right)$ следует вычислить либо $H_{1}\left(\mathscr{J}\left(\mathbb{Z}_{p}\right), \mathbb{Z}\right)$, либо $H^{1}\left(\mathscr{J}\left(\mathbb{Z}_{p}\right), \mathbb{Z}_{p}\right)$. В случае нечетного $p$ простое вычисление показывает, что обе группы изоморфны $\mathbb{Z}_{p} \oplus \mathbb{Z}_{p}$. Если $p=2$, то несколько более сложное вычисление приводит к равенству $H_{1}\left(\mathscr{J}\left(\mathbb{Z}_{2}\right), \mathbb{Z}\right)=\mathbb{Z}_{2} \oplus \mathbb{Z}_{4} \oplus \mathbb{Z}_{2}$, отсюда легко находим, что $H^{1}\left(\mathscr{J}\left(\mathbb{Z}_{2}\right), \mathbb{Z}_{2}\right)=$ 
$\mathbb{Z}_{2} \oplus \mathbb{Z}_{2} \oplus \mathbb{Z}_{2}$. Порождаемость $\mathscr{J}\left(\mathbb{Z}_{p}\right)$ приведенными наборами образующих может быть доказана прямым индуктивным вычислением $\bmod \mathscr{J}^{n}\left(\mathbb{Z}_{p}\right)$.

Рассмотрим, какие вообще подгруппы в $\mathscr{J}\left(\mathbb{Z}_{p}\right)$ могут порождаться семейством $f$ образующих из теоремы 4.5. Обозначим $\mathbf{m}(p)$ вероятностную меру Хаара на группе $\mathscr{J}\left(\mathbb{Z}_{p}\right)$, она имеет прозрачный геометрический смысл. Так, мера любого подмножества вида

$$
A\left(\alpha_{1}, \alpha_{2}, \ldots, \alpha_{n}\right)=\left\{f(x)=x\left(1+\alpha_{1} x+\alpha_{2} x^{2}+\cdots+\alpha_{n} x^{n}\right)+\mathscr{O}\left(x^{n+2}\right)\right\},
$$

где $\alpha_{1}, \alpha_{2}, \ldots, \alpha_{n}$ фиксированы, равна

$$
\mathbf{m}(p)\left(A\left(\alpha_{1}, \alpha_{2}, \ldots, \alpha_{n}\right)\right)=\frac{1}{p^{n}} .
$$

Подмножества $A\left(\alpha_{1}, \alpha_{2}, \ldots, \alpha_{n}\right)$ открыто-замкнуты и порождают борелевскую $\sigma$-алгебру на $\mathscr{J}\left(\mathbb{Z}_{p}\right)$. Единственное продолжение меры (23) на полную борелевскую $\sigma$-алгебру и дает нам требуемую вероятностную меру Хаара на $\mathscr{J}\left(\mathbb{Z}_{p}\right)$. Заметим, что полученная таким образом мера совпадает с колмогоровским пределом (см. [88]), соответствующим обратной последовательности (11), дискретных вероятностных мер на конечных группах $\mathscr{J}_{n}\left(\mathbb{Z}_{p}\right)$. Следующий результат Сегеди [104] представляет отдельный интерес.

ТЕОРема 4.6. Любъе $n$ элементов группь $\mathscr{J}\left(\mathbb{Z}_{p}\right)$ почти наверное порождают свободную подгруппу ранга $n$.

СлЕДСТвИЕ 4.7. Образующие $f_{1}, f_{2}$ группъ $\mathscr{J}\left(\mathbb{Z}_{p}\right), p>2\left(f_{1}, f_{2}, f_{6}\right.$ в случае $p=2)$, при случайном выборе остаточных членов почти наверное порождают свободную подгруппу ранга 2 (ранга 3 в случае $p=2)$.

Эта теорема показывает, что с точки зрения поведения случайных конечно порожденных подгрупп группы $\mathscr{J}\left(\mathbb{Z}_{p}\right)$ ведут себя похоже на группы Ли, не являющиеся разрешимыми [39]. Несмотря на то что любая случайная подгруппа группы $\mathscr{J}\left(\mathbb{Z}_{p}\right)$ свободна, в этой группе существуют многочисленные соотношения между элементами - приведем один нетривиальный пример, содержащийся в [60].

ПримеР 4.8. Рассмотрим следующие четыре элемента группы $\mathscr{J}(\mathbb{Z})$ :

$$
\begin{array}{ll}
u_{2}(x)=x+27 x^{2}, & v_{3}(x)=x+32 x^{2}+256 x^{3}, \\
u_{3}(x)=x+8 x^{2}+16 x^{3}, & v_{2}(x)=x+3 x^{3} .
\end{array}
$$

Оставляем читателю проверку равенства

$$
u_{2} \circ u_{3}=v_{3} \circ v_{2} .
$$

Это соотношение справедливо в группе $\mathscr{J}(\mathbb{Z})$. Делая в $(24)$ редукцию коэффициентов $\bmod p$, мы получим четыре элемента в $\mathscr{J}\left(\mathbb{Z}_{p}\right)$, которые удовлетворяют тому же самому соотношению. Если $p \neq 2,3$, то это дает нетривиальные примеры в $\mathscr{J}\left(\mathbb{Z}_{p}\right)$. 
Несмотря на то что образующие теоремы 4.5 почти наверное порождают свободную группу, $\mathscr{J}\left(\mathbb{Z}_{p}\right)$, как про- $p$-группа, может быть задана конечным набором соотношений. Напомним необходимые определения (см. [96] или [110]). Пусть $f=\left\{f_{1}, f_{2}, \ldots, f_{n}\right\}-$ (конечный) набор символов и $F(f)$ - порожденная этим набором свободная группа. Свободной про-р-группой $\mathscr{F}(f)$, порожденной $f$, называется про- $p$-пополнение свободной группы $F(f)$. Группу $\mathscr{F}(f)$ можно представить следующим образом. Рассмотрим множество пар $(M, \phi)$, где $M$ - конечная $p$-группа, а $\phi: F(f) \rightarrow M$ - эпиморфизм. Имеется естественный мономорфизм

$$
\Phi=\prod_{(M, \phi)} \phi: F(f) \rightarrow \prod_{(M, \phi)} M=\mathscr{M} .
$$

Замыкание образа $\Phi(F(f))$ в про-р-группе $\mathscr{M}$ и будет группой $\mathscr{F}(f)$.

Пусть теперь $G$ - некоторая про-р-группа, порожденная семейством образующих $f$. Имеется естественный эпиморфизм про- $p$-групп

$$
\mathscr{F}(f) \rightarrow G
$$

ядро которого обозначим $\mathscr{R}$. Говорят, что элементы $r=\left\{r_{1}, r_{2}, \ldots, r_{m}\right\}$ порождают $\mathscr{R}$ (как нормальный делитель в $\mathscr{F}(f)$ ), если наименьшая замкнутая нормальная подгруппа в $\mathscr{F}(f)$, содержащая $r$, совпадает с $\mathscr{R}$. В этом случае говорят, что группа $G$ может быть задана не более чем $m$ соотношениями. Наименьшее возможное число $m$ элементов в семействе $r$ называется рангом $\mathscr{R}$. Следующий результат Ершова [42] устанавливает конечную заданность $\mathscr{J}\left(\mathbb{Z}_{p}\right)$ при нечетном $p$.

Теорема 4.9. Если $p$ - нечетное простое число, то группа $\mathscr{J}\left(\mathbb{Z}_{p}\right)$ может быть задана не более чем $12 p+32$ соотношениями.

Конечная заданность $\mathscr{J}\left(\mathbb{Z}_{2}\right)$ остается полностью неизученной. Также не известно никакой универсальной (не зависящей от характеристики $p$ ) оценки на число соотношений. В работе [42] рассматриваются ассоциированные с $\mathscr{J}\left(\mathbb{Z}_{p}\right)$ градуированные $\mathbb{Z}_{(p)}$-алгебры Ли. Исследование их конечной представимости и приводит к указанной выше оценке. Между тем (см. [96] или [110]), если про- $p$-группа $G$ задана $n$ образующими и имеет конечное число соотношений, то для минимального количества соотношений $m$ справедливо равенство

$$
m=n-b_{1}+b_{2}
$$

где $b_{1}$ и $b_{2}-$ первое и второе $\mathbb{Z}_{p}$-числа Бетти группы $G$. Таким образом, вопрос о точной оценке числа соотношений в $\mathscr{J}\left(\mathbb{Z}_{p}\right)$ помогло бы решить вычисление когомологий $H^{2}\left(\mathscr{J}\left(\mathbb{Z}_{p}\right), \mathbb{Z}_{p}\right)$. Результаты в этом направлении автору не известны.

4.4. Универсальность группы $\mathscr{J}\left(\mathbb{Z}_{p}\right)$. Как мы видели, $\mathscr{J}\left(\mathbb{Z}_{p}\right)$ является про-р-группой со счетной базой. Оказывается, что $\mathscr{J}\left(\mathbb{Z}_{p}\right)$ обладает свойством универсальности для про- $p$-групп со счетной базой. Оставшуюся часть этого пункта посвятим следующему результату, впервые доказанному Каминой [23]. 
ТеОрема 4.10. Всякая про-р-группа со счетной базой вкладьвается в групny $\mathscr{J}\left(\mathbb{Z}_{p}\right)$.

ЗАмЕчАНИЕ 4.11. В п. 3.1 были описаны все подгруппы $\mathscr{J}\left(\mathbb{Z}_{p}\right)$, изоморфные $\mathbb{Z}_{p}$. Все эти подгруппы были реализованы, с точностью до сопряжения, явными вложениями. Из теоремы 4.10 , в частности, вытекает существование вложений $\mathbb{Z}_{p} \oplus \mathbb{Z}_{p}$ и $\mathbb{Z}_{p^{2}}$ в $\mathscr{J}\left(\mathbb{Z}_{p}\right)$. Удивительно, но ни одного явного такого вложения не известно! Например, было бы очень интересно найти элемент порядка $p^{2}$ в группе $\mathscr{J}\left(\mathbb{Z}_{p}\right)$, который в ней заведомо существует.

Проблема вложимости различных классов групп в соответствующую универсальную группу известна давно и этой тематике посвящен целый ряд работ, среди которых, не претендуя на полноту списка, мы отметим лишь [84], [109], [72]. Приведем основной результат, принадлежащий Любоцкому и Вильсону [72] и представляющий независимый интерес. Сформулированное ниже следствие из этого результата существенным образом используется в оригинальном доказательстве [23] теоремы 4.10.

ТЕОРема 4.12. Пусть $\mathscr{C}$ - класс конечных групп, замкнутый относительно взятия подгрупп и расширений. Существует про-С્C-группа с двумя образующими, в которую может быть вложена любая про-С્С-группа со счетной базой.

Из этой теоремы непосредственно вытекает следующий результат.

СЛЕДСТВИЕ 4.13. Существует универсальная про-р-группа с двумя образующими, в которую вкладывается любая про-р-группа со счетной базой.

Универсальные группы Любоцкого-Вильсона допускают вполне конструктивное описание, но все-таки являются специально сконструированными объектами. Несомненное преимущество теоремы 4.10 - доказательство универсальности естественного объекта, группы $\mathscr{J}\left(\mathbb{Z}_{p}\right)$.

Чтобы составить представление об идеях, задействованных в доказательстве теоремы 4.10, дадим здесь его набросок, подробности можно найти в работе [23].

Рассмотрим локальное поле $\mathbb{Z}_{p}((x))$ (для тех же целей можно было бы рассматривать локальное кольцо $\left.\mathbb{Z}_{p}[[x]]\right)$. Группа (непрерывных) автоморфизмов $\mathbb{Z}_{p}((x))$ изоморфна группе формальных рядов $\mathscr{A}\left(\mathbb{Z}_{p}\right)$. Если $h(x) \in \mathbb{Z}_{p}((x))$ и $f(x) \in \mathscr{A}\left(\mathbb{Z}_{p}\right)$, то действие $\mathscr{A}\left(\mathbb{Z}_{p}\right)$ на $\mathbb{Z}_{p}((x))$ задается как $h(x) \mapsto h(f(x))$. Группа $\mathscr{J}\left(\mathbb{Z}_{p}\right)$ нормальна в $\mathscr{A}\left(\mathbb{Z}_{p}\right)$ и имеет место короткая точная последовательность

$$
1 \longrightarrow \mathscr{J}\left(\mathbb{Z}_{p}\right) \longrightarrow \mathscr{A}\left(\mathbb{Z}_{p}\right) \longrightarrow \mathbb{Z}_{p-1} \longrightarrow 1,
$$

см. замечание 2.23. Здесь $\mathbb{Z}_{p-1}$ рассматривается как мультипликативная подгруппа поля $\mathbb{Z}_{p}$. Центральную роль в последующей части доказательства играет следующий классический результат Витта [112] 30-х годов прошлого века.

Теорема 4.14. Пусть $\mathbf{k}$ - поле характеристики р и $G$ - конечнал р-группа. Существует расширение $\mathbf{K}$ поля $\mathbf{k}$ такое, что группа Галуа $\mathrm{Gal}(\mathbf{K}: \mathbf{k})$ изоморфна $G$. 
К сожалению, доказательство существования такого расширения неявно и не дает возможности конструктивно восстановить К. Сперва Витт решает проблему для абелевых $p$-групп $G$, строя некоторое абелево расширение. Затем, некоторым итерационным процессом, он строит $\mathbf{K}$ в случае общей $p$-группы $G$.

Анализируя эту конструкцию Витта, Лидхем-Грин и Вайс (не опубликовано) заметили, что если начальное поле $\mathbf{k}$ есть $\mathbb{Z}_{p}((x))$, то полученное расширение $\mathbf{K}$ можно выбрать вполне разветвленным накрытием поля $\mathbb{Z}_{p}((x))$. Известно, что в этом случае оно должно быть изоморфно исходному полю $\mathbb{Z}_{p}((x))$. Иными словами, в рассматриваемом случае группа Галуа $G$ реализуется как группа автоморфизмов поля $\mathbb{Z}_{p}((x))$, т. е. $G \subset \mathscr{A}\left(\mathbb{Z}_{p}\right)$. Поскольку $G-p$-группа, а $\mathscr{A}\left(\mathbb{Z}_{p}\right) / \mathscr{J}\left(\mathbb{Z}_{p}\right) \simeq Z_{p-1}$, то получаем, что $G \subset \mathscr{J}\left(\mathbb{Z}_{p}\right)$.

Следствие 4.13 позволяет свести проблему вложения общих про-р-групп со счетной базой к вопросу вложения одной специальной про- $p$-группы. В несколько более общем виде можно сказать, что достаточно научиться вкладывать в $\mathscr{J}\left(\mathbb{Z}_{p}\right)$ лишь конечно порожденные про-р-группы. Этот последний этап был осуществлен Каминой [23], которая, используя трюк Витта в случае локального поля $\mathbb{Z}_{p}((x))$, получила следующий результат.

ТеОрема 4.15. Любая конечно порожденная про-р-группа может быть вложена в группу $\mathscr{J}\left(\mathbb{Z}_{p}\right)$.

Для завершения доказательства теоремы 4.10 остается применить этот результат к универсальной про-р-группе следствия 4.13.

ЗАмечАниЕ 4.16. Вскорости после работы Камины [23] альтернативное доказательство теоремы 4.10 было дано Фесенко [48]. Доказательство этой работы непосредственно использует теорию Галуа расширений локальных полей и не использует следствие 4.13. Это позволяет получить само следствие 4.13 как побочный результат, взяв в качестве универсальной про- $p$-группы с двумя образующими саму группу $\mathscr{J}\left(\mathbb{Z}_{p}\right)$. Здесь следует отметить, что это соображение справедливо лишь при $p>2$, поскольку, как мы видели выше, группа $\mathscr{J}\left(\mathbb{Z}_{2}\right)$ имеет не менее 3 образующих. О связи теории Галуа расширений локальных полей с группой $\mathscr{J}\left(\mathbb{Z}_{p}\right)$ см. также [33].

\section{5. Дальнейшие топологические свойства групп $\mathscr{J}\left(\mathbb{Z}_{p}\right)$ и некото-} рых групп $\mathscr{J}(\mathbf{k})$. Изучение замкнутых подгрупп, в частности замкнутых нормальных делителей, в некоторой топологической группе доставляет важную информацию о ее строении. Изучение таких подгрупп в $\mathscr{J}\left(\mathbb{Z}_{p}\right)$, кроме внутреннего интереса, позволяет еще ответить на некоторые старые вопросы теории про- $p$-групп.

Оказывается, что для многих нётеровых колец $\mathbf{k}$ нормальные подгруппы в $\mathscr{J}(\mathbf{k})$ автоматически замкнуты и жестко вложены. Начнем со следующего замечания, которое объясняет механизм, отвечающий за справедливость дальнейших результатов.

ЗАмЕчАНИЕ 4.17. Пусть $H \unlhd \mathscr{J}\left(\mathbb{Z}_{p}\right)$ - замкнутый нормальный делитель, а $p$ - нечетное простое число. Очевидно, найдется максимальное натуральное число $n$ такое, что $H \subseteq \mathscr{J}^{n}\left(\mathbb{Z}_{p}\right)$. Рассматривая коммутаторы $\left[f_{1}, g\right]$ или $\left[f_{2}, g\right]$, 
где $f_{1}$ и $f_{2}$ - образующие $\mathscr{J}\left(\mathbb{Z}_{p}\right)$, а $g \in H$, несложно показать, что $\mathscr{J}^{n+1}\left(\mathbb{Z}_{p}\right) \subseteq$ $H$, если $n \neq k p+1$, и $\mathscr{J}^{n+2}\left(\mathbb{Z}_{p}\right) \subseteq H$ в противном случае. Рекомендуем читателю провести соответствующие вычисления.

Аналогичное свойство замкнутых нормальных подгрупп верно и в любой группе $\mathscr{J}(\mathbf{k})$, где $\mathbf{k}-($ не обязательно конечное) поле характеристики $p \neq 2$. Более того, имеет место следующее существенное обобщение, доказанное Клопшем [66].

ТеОРема 4.18. Пусть $\mathbf{k}$ - поле характеристики $p>2$. Любая (абстрактная) нормальная подгруппа $H \unlhd \mathscr{J}(\mathbf{k})$ замкнута. Если $n$ - максимальное натуральное число такое, что $H \subseteq \mathscr{J}^{n}\left(\mathbb{Z}_{p}\right)$, mо $\mathscr{J}^{n+1}(\mathbf{k}) \subseteq H \subseteq \mathscr{J}^{n}(\mathbf{k})$, если $n \neq k p+1, u \mathscr{J}^{n+2}(\mathbf{k}) \subseteq H \subseteq \mathscr{J}^{n}(\mathbf{k})$, если $n=k p+1$.

В частности, эта теорема показывает, что если $\mathbf{k}$ конечно, то любой нормальный делитель в $\mathscr{J}(\mathbf{k})$ имеет конечный индекс. Напомним, что топологическая группа $G$ называется едва бесконечной, если любая ее нетривиальная замкнутая нормальная подгруппа имеет конечный индекс. Соответственно группа $G$ называется наследственно едва бесконечной, если любая ее открытая подгруппа является едва бесконечной.

Используя теорему 4.18 и тот факт, что группы $\left\{\mathscr{J}^{n}(\mathbf{k})\right\}_{n=1}^{\infty}$ образуют базу окрестностей единицы, несложно получить, что группа $\mathscr{J}(\mathbf{k})$ наследственно едва бесконечна для любого конечного поля характеристики $p \neq 2$.

Следующий результат Хегедюса [54] требует еще более тонких вычислений, чем необходимы для доказательства теоремы 4.18 и тем более для обоснования замечания 4.17 .

ТЕОРема 4.19. Для любого конечного поля $\mathbf{k}=\mathbb{F}_{2^{d}}$ характеристики 2 группа $\mathscr{J}(\mathbf{k})$ наследственно едва бесконечна.

Идея доказательства состоит в рассмотрении некоторого неединичного элемента $h$ в открытой подгруппе $H \subseteq \mathscr{J}(\mathbf{k})$ и доказательства открытости его нормального замыкания $\langle h\rangle^{H}$, детали см. в [54].

Теорема 4.18 утверждает, в частности, автоматическую замкнутость нормальных подгрупп $\mathscr{J}(\mathbf{k})$ в случае поля характеристики $p \neq 2$. Оказывается, что свойство замкнутости верно в гораздо более общей ситуации.

Рассмотрим (дискретное) кольцо с единицей $\mathbf{k}$ и пусть $I \subset \mathbf{k}-$ идеал. Подгруппа $\mathscr{J}(I) \subseteq \mathscr{J}(\mathbf{k})$ нормальна (п. 2.2) и замкнута; положим $\mathscr{J}^{n}(I)=$ $\mathscr{J}(I) \cap \mathscr{J}^{n}(\mathbf{k})$. Далее, для любой подгруппы $H \subseteq \mathscr{J}(k)$ определим подмножество "младших элементов", положив

$$
M(H)=\left\{\alpha \in \mathbf{k} \mid x\left(1+\alpha x^{n}+\mathscr{O}\left(x^{n+1}\right)\right) \in H \text { для некоторого натурального } n\right\} .
$$

Пусть $I(H)=I(M(H))$ - порожденный этим подмножеством идеал в $\mathbf{k}$. Имеет место следующий общий результат Клопша [66].

ТеОрема 4.20. Пусть $\mathbf{k}$ - нётерово коммутативное кольцо с единицей, в котором 2 - обратимый элемент. Тогда любая абстрактная нормальная подгруппа $H \unlhd \mathscr{J}(\mathbf{k})$ замкнута. Более того, если $H \subseteq \mathscr{J}(I(H))$, то найдется натуральное $n$ такое, что $\mathscr{J}^{n}(I(H)) \subseteq H$. 
Насколько условие включения $H \subseteq \mathscr{J}(I(H))$ независимо, не ясно. В цитированной выше работе [66] ставится вопрос:

Могут ли в предположениях теоремы на $\mathrm{k}$ существовать замкнутые нормальные подгруппы $H \unlhd \mathscr{J}(\mathbf{k})$ такие, что $H \nsubseteq \mathscr{J}(I(H))$ ?

Неясно также, существенно ли ограничение на характеристику в теореме 4.20. В этой связи в [66] поставлен второй естественный вопрос:

Пусть $\mathbf{k}$ - нётерово коммутативное кольцо с единицей, в котором 2 необратима (или даже $2=0$ ), существуют ли незамкнутые нормальные подгруппь $H \unlhd \mathscr{J}(\mathbf{k})$ ?

Насколько известно автору, ответов на эти вопросы пока нет.

Если $H \subseteq \mathscr{J}(\mathbf{k})$ - открытая подгруппа, то $\mathscr{J}^{n}(\mathbf{k}) \subset H$ для подходящего номера $n$. Связь между минимальным допустимым $n$ и индексом $H$ определяется следующим результатом.

ТЕОРЕма 4.21. Пусть $\mathbf{k}=\mathbb{F}_{q}$ конечное поле характеристики $p$ u $\mathrm{C}$ $\mathscr{J}(\mathbf{k})$ - открытая подгруппа индекса $p^{k}$. Тогда $\mathscr{J}^{n}(\mathbf{k}) \subseteq H$, где $n=\left[\frac{2 k p}{p-1}\right]$, если $p>2, u n=6 k+3$, если $p=2$.

Случай нечетной характеристики доказан Лидхэм-Грином и Шалевым (не опубликовано, см. [25]), в характеристике 2 результат установлен Хегедюсом [54].

Разнообразие открытых подгрупп некоторой проконечной группы $G$ отражает группа ее абстрактных соизмерений $\operatorname{Comm}(G)$. Кратко определим этот объект, систематическое изучение которого было начато в [11]. Виртуальным автоморфизмом группы $G$ называется некоторый изоморфизм между двумя ее открытыми подгруппами. Будем говорить, что два виртуальных автоморфизма эквивалентны, если они совпадают на некоторой открытой подгруппе. Множество классов эквивалентности виртуальных автоморфизмов и есть $\operatorname{Comm}(G)$. Несложно проверить, что это группа, причем имеется естественный гомоморфизм

$$
\rho: \operatorname{Aut}(G) \rightarrow \operatorname{Comm}(G) .
$$

Вообще говоря, гомоморфизм (25) имеет нетривиальное ядро и коядро. Оказывается, что группа $\mathscr{J}\left(\mathbb{Z}_{p}\right)$ обладает соответствующей жесткостью. Следующий результат установлен Ершовым [44].

ТеОрема 4.22. Если р - простое число, не менъшее 5, то естественное отображение

$$
\rho: \operatorname{Aut}\left(\mathscr{J}\left(\mathbb{Z}_{p}\right)\right) \rightarrow \operatorname{Comm}\left(\mathscr{J}\left(\mathbb{Z}_{p}\right)\right)
$$

является изоморфизмом.

Таким образом, $\mathscr{J}\left(\mathbb{Z}_{p}\right) \subset \operatorname{Comm}\left(\mathscr{J}\left(\mathbb{Z}_{p}\right)\right)$ - нормальная подгруппа индекса $p-1$ (см. теорему 2.24).

4.6. Индексные и некоторые другие большие подгруппы. Если приведенные в предыдущем пункте результаты в достаточной степени проясняют структуру замкнутых нормальных подгрупп в $\mathscr{J}(\mathbf{k})$, где $\mathbf{k}=\mathbb{F}_{q}$ - конечное поле характеристики $p$, то наше представление о произвольных замкнутых подгруппах довольно туманно. 
Начнем с нескольких общих простых замечаний. Если $\mathbf{k}$ - компактное коммутативное кольцо с единицей, то замкнутая циклическая подгруппа $\mathscr{J}(\mathbf{k})$ конечна. Если $\mathbf{k}$ - конечное кольцо, то любая бесконечная замкнутая подгруппа $\mathscr{J}(\mathbf{k})$ гомеоморфна канторову множеству. Если $f \in \mathscr{J}(\mathbf{k})$ - элемент бесконечного порядка, то замыкание порожденной им подгруппы $\overline{\left\{f^{k}\right\}_{k=-\infty}^{\infty}}-$ проциклическая подгруппа в $\mathscr{J}(\mathbf{k})$.

Теорема 4.1 дает исчерпывающее описание конечных подгрупп порядка $p$ в $\mathscr{J}\left(\mathbb{F}_{p^{d}}\right)$. С другими замкнутыми циклическими подгруппами, имеющими, очевидно, порядок $p^{k}, k>1$, ситуация совершенно неясна. Хотя, согласно теореме 4.10, такие подгруппы существуют для всех $k>1$, ни одного явного примера этих подгрупп не известно. Циклические, проциклические, конечные и многие другие подгруппы в $\mathscr{J}(\mathbf{k})$ относятся к так называемым малым подгруппам. Чтобы определить сравнение подгрупп по размеру, рассмотрим ситуацию общей проконечной группы, см. также [13].

Пусть $G$ - проконечная группа. Рассмотрим фильтрацию $\Phi=\left\{G_{n}\right\}_{n=1}^{\infty}$ на $G$, т. е. убывающую последовательность открытых нормальных подгрупп, образующую базу окрестностей единицы. На $G$ можно определить инвариантную ультраметрику, полагая

$$
\rho_{\Phi}(f, g)=\frac{1}{\left|G: G_{n}\right|}, \quad \text { где } f, g \in G, \quad \text { причем } f \circ g^{-1} \in G_{n} \quad \text { и } f \circ g^{-1} \notin G_{n+1} \text {. }
$$

Введенная так метрика зависит от выбранной на $G$ фильтрации $\Phi$. Тем не менее она позволяет использовать метрические инварианты, например хаусдорфову размерность, для изучения и характеризации подгрупп в $G$. Замкнутую подгруппу в $G$ удобно называть малой, если она имеет нулевую хаусдорфову размерность, и большой, если ее хаусдорфова размерность положительна. Как и метрика (26), введенная с ее помощью хаусдорфова размерность может зависеть от выбора фильтрации $\Phi$.

Определенную выше хаусдорфову размерность замкнутой подгруппы $H \leqslant G$ относительно выбранной фильтрации $\Phi$ можно вычислить по чисто алгебраической формуле

$$
\operatorname{hdim}(H)=\liminf _{n \rightarrow \infty} \frac{\ln \left|H G_{n}: G_{n}\right|}{\ln \left|G: G_{n}\right|} .
$$

Хаусдорфовым спектром проконечной группы $G$ назовем следующее подмножество единичного интервала:

$$
\operatorname{hspec}(G)=\{\operatorname{hdim}(H) \mid H-\text { замкнутая подгруппа в } G\} .
$$

Несложно видеть, что $\operatorname{hdim}(H)=1$ для любой открытой подгруппы $H \leqslant G$. Таким образом, хаусдорфов спектр некоторой проконечной группы $G$ характеризует разнообразие ее замкнутых подгрупп. При этом он, естественно, не характеризует подгруппы с точностью до изоморфизма, одна и та же группа может быть вложена в $G$ с разными хаусдорфовыми размерностями. Спектр (27), вообще говоря, зависит от выбора фильтрации в $G$. Для групп $\mathscr{J}(\mathbf{k})$ естественно использовать фильтрацию подгруппами $\left\{\mathscr{J}^{n}(\mathbf{k})\right\}_{n=1}^{\infty}$.

Удачный подкласс больших замкнутых подгрупп в $\mathscr{J}(\mathbf{k})$ составляют так называемые индекснъе подгруппы. Отдельные примеры таких подгрупп были 
известны довольно давно. Систематическое изучение этого класса было предпринято Барнеа и Клопшем в [12], ниже мы во многом следуем обозначениям и определениям этой работы.

Пусть $\mathbf{k}$ - коммутативное кольцо с единицей и $L \subset \mathbb{N}$ - подмножество натуральных чисел. Определим замкнутое подмножество $\mathscr{J}(\mathbf{k})$, положив

$$
\mathscr{J}_{L}(\mathbf{k})=\left\{x\left(1+\sum_{i \in L} \alpha_{i} x^{i}\right) \mid a_{i} \in \mathbf{k}, i \in L\right\} .
$$

В случае, когда подмножество $\mathscr{J}_{L}(\mathbf{k})$ оказывается группой, будем говорить, что $L$ - допустимое индексное множество, а саму группу $\mathscr{J}_{L}(\mathbf{k})$ будем называть индексной подгруппой, отвечающей $L$. Общая структура индексных подгрупп в случае поля конечной характеристики была описана в [12].

Теорема 4.23. Пусть $\mathbf{k}$ - конечное поле характеристики $p$ u $L \subseteq \mathbb{N}$. Подмножество $\mathscr{J}_{L}(\mathbf{k}) \subseteq \mathscr{J}(\mathbf{k})$ является (замкнутой) подгруппой тогда и только тогда, когда для любого $i \in L$ u $n \in\{1,2, \ldots, i+1\}$ такого, ито $\left(\begin{array}{c}i+1 \\ n\end{array}\right) \neq 0$ $\bmod p$, имеет место включение $\{i+n j \mid j \in L\} \subseteq L$.

Сравнительно короткое доказательство этого результата, использующее тонкие и элегантные вычисления, дано в [54]. Следующий пример [12] показывает возможное разнообразие индексных подгрупп, мы сохраняем авторские обозначения.

ПримеР 4.24. Следующие подмножества являются индексными подгруппами в $\mathscr{J}(\mathbf{k})$ :

a) $\mathscr{A}_{s}=\mathscr{J}_{[s \mathbb{N}]}(\mathbf{k})$, где $s \in \mathbb{N}$;

b) $\mathscr{B}_{r, s}=\mathscr{J}_{\left[p^{r} \mathbb{N} \cup\left(p^{s} \mathbb{N}-1\right)\right]}\left(\mathbb{Z}_{p}\right)$, где $r, s \in \mathbb{N}$ и $r \geqslant s$;

c) $\mathscr{C}_{s}=\mathscr{J}_{\left[p^{s}-1\right]}\left(\mathbb{Z}_{p}\right)$, где $s \in \mathbb{N}$;

d) $\mathscr{D}=\mathscr{J}\left[\left\{p^{n}-1\right\}_{n=1}^{\infty}\right]\left(\mathbb{Z}_{p}\right)$.

Проверка того, что подмножества из этого примера являются индексными подгруппами, может быть неплохим упражнением.

Легко видеть, что семейство подгрупп $\mathscr{A}_{s}$ совпадает с рассмотренным в п. 2.3 семейством $\mathscr{J}_{(s)}(\mathbf{k})$. Группы этого семейства $\mathscr{A}_{s}$ изучались в [24] в случае простого поля характеристики $p$. Заметим также, что семейство $\mathscr{A}_{s}$ отличается от остальных тем, что эти индексные подгруппы имеют смысл для любого основного кольца $\mathbf{k}$.

Предложение 3.8 показывает, что если $\mathbf{k}$ дискретно, а $s$ обратим в $\mathbf{k}$, то имеет место изоморфизм $\mathscr{A}_{s} \simeq \mathscr{J}(\mathbf{k})$. В случае поля конечной характеристики, в частности $\mathbf{k}=\mathbb{Z}_{p}$, подкласс $\mathscr{A}_{m p}$ представляет самостоятельный интеpec. Несложно показать, что в этом случае группы $\mathscr{A}_{m p}$ свободны от кручения и, следовательно, не изоморфны группе $\mathscr{J}\left(\mathbb{Z}_{p}\right)$. Как показал Фесенко в [48], $\mathscr{A}_{m p}$ является наследственно едва бесконечной группой, но не является $p$-адически аналитичной группой. В этой же работе (см. также [33]) рассмотрены интересные приложения групп $\mathscr{A}_{p^{r}}$ в теории чисел, этот круг вопросов мы здесь не обсуждаем. 
Рассмотрим еще один пример из работы [12], представляющий самостоятельный интерес.

ПримеР 4.25. Пусть $U_{1}=\left\{1+x \mathbb{Z}_{p}[[x]]\right\} \subset \mathbb{Z}_{p}[[x]]-$ специальная подгруппа обратимых элементов в кольце формальных степенных рядов с коэффициентами $\mathbb{Z}_{p}$. Отображение

$$
\varphi\left(x+\sum_{n=1}^{\infty} \alpha_{n} x^{p^{n}}\right)=1+\sum_{n=1}^{\infty} \alpha_{n} x^{n}
$$

задает изоморфизм про- $p$-групп $\mathscr{D}$ и $U_{1}$. Таким образом, $\mathscr{D}$ - свободная абелева про-р-группа бесконечного ранга. О связи группы $\mathscr{D}$ с мультипликативными когомологическими операциями в $H^{*}\left(, \mathbb{Z}_{p}\right)$-теории см. теорему 7.10 .

В связи с этим примером отметим общее ограничение, найденное в [111], которому подчиняются абелевы подгруппы в $\mathscr{J}\left(\mathbb{Z}_{p}\right)$.

Теорема 4.26. Пусть $H \subset \mathscr{J}\left(\mathbb{Z}_{p}\right)$ - абелева подгруппа, положим

$$
I=\left\{i \in \mathbb{N} \mid H \cap \mathscr{J}^{i}\left(\mathbb{Z}_{p}\right) \neq H \cap \mathscr{J}^{i+1}\left(\mathbb{Z}_{p}\right)\right\}=\left\{i_{1}<i_{2}<\cdots\right\} .
$$

Тогда $i_{n+1} \equiv i_{n} \bmod p^{n}$ для всех $n \in \mathbb{N}$.

Интересную информацию о разнообразии и строении индексных подгрупп дает следующий результат [12].

Tеорема 4.27. а) Подгруппа $\mathscr{C}_{1} \leqslant \mathscr{J}\left(\mathbb{Z}_{p}\right)$ содержит несчетное множество индексных подгрупп, которые не являются конечно порожденными.

b) Любая не содержащаяся в $\mathscr{C}_{1}$ индексная подгруппа конечно порождена.

c) Подгруппь $\mathscr{B}_{r, r}, r \in \mathbb{N}$, попарно несоизмеримы.

В случае $G=\mathscr{J}\left(\mathbb{Z}_{p}\right)$ в работе [12] вводится подмножество $\operatorname{inspec}\left(\mathscr{J}\left(\mathbb{Z}_{p}\right)\right) \subseteq$ $\operatorname{hspec}\left(\mathscr{J}\left(\mathbb{Z}_{p}\right)\right)$, называемое индексным спектром, которое получается из $(27)$, если $H$ пробегает только индексные подгруппы в $\mathscr{J}\left(\mathbb{Z}_{p}\right)$. Следующий результат работы [12] дает точное вычисление inspec $\left(\mathscr{J}\left(\mathbb{Z}_{p}\right)\right)$ и проясняет структуру хаусдорфова спектра этой группы.

Теорема 4.28. Относительно канонической фильтрации $\left\{\mathscr{J}^{n}\left(\mathbb{Z}_{p}\right)\right\}_{n=1}^{\infty}$ группы $\mathscr{J}\left(\mathbb{Z}_{p}\right)$ имеет место равенство

$$
\operatorname{inspec}\left(\mathscr{J}\left(\mathbb{Z}_{p}\right)\right)=\left[0, \frac{1}{p}\right] \cup\left\{\frac{1}{p}+\frac{1}{p^{r}}\right\}_{r=1}^{\infty} \cup\left\{\frac{1}{s}\right\}_{s=1}^{\infty} .
$$

ЗАмЕчАнИЕ 4.29 . Если $p>2$, то результат теоремы 4.28 не зависит от выбора фильтрации в $\mathscr{J}\left(\mathbb{Z}_{p}\right)$.

Неожиданным следствием теоремы 4.28 является наличие интервала в окрестности нуля в хаусдорфовом спектре группы $\mathscr{J}\left(\mathbb{Z}_{p}\right)$. Мы видим, что $\mathscr{J}\left(\mathbb{Z}_{p}\right)$ имеет огромный запас больших подгрупп. Отметим, что, согласно [43], конечно порожденная большая подгруппа в $\mathscr{J}\left(\mathbb{Z}_{p}\right)$ не может быть линейной группой над локальным полем. Работа [12] содержит ряд других результатов об индексных подгруппах в $\mathscr{J}\left(\mathbb{Z}_{p}\right)$, в этом обзоре мы их не обсуждаем, отсылая интересующихся к оригинальной статье. 
Другой класс отличных от индексных больших подгрупп в $\mathscr{J}\left(\mathbb{Z}_{p}\right)$ был найден Ершовым [41], этот класс представляет самостоятельный интерес. Пусть $p$ - нечетное простое число, а $r$ и $s$ - натуральные числа, удовлетворяющие неравенству $r<p^{s} / 2$, причем $p$ и $r$ взаимно просты. Рассмотрим подмножество $\mathscr{Q}^{1}(r, s) \subset \mathscr{J}\left(\mathbb{Z}_{p}\right)$, состоящее из рядов вида

$$
f(x)=\left(\frac{a x^{r}+b}{c x^{r}+d}\right)^{1 / r},
$$

где $a, b, c, d \in \mathbb{Z}_{p}\left[\left[x^{p^{s}}\right]\right]$, причем $a_{0}=d_{0}=1$ и $b_{0}=c_{0}=0$. Несложно проверить, что $\mathscr{Q}^{1}(r, s)$ - замкнутая подгруппа в $\mathscr{J}\left(\mathbb{Z}_{p}\right)$. Несколько бо́льшая подгруппа $\mathscr{Q}(r, s) \subset \mathscr{J}\left(\mathbb{Z}_{p}\right)$ состоит из рядов вида $(28)$ при отброшенном условии $c_{0}=0$. Группу $\mathscr{Q}(r, s)$ можно представить как некоторую силовскую про-р-подгруппу в $\mathrm{SL}_{2}\left(\mathbb{Z}_{p}[[t]]\right)$, при этом $\mathscr{Q}^{1}(r, s)$ - подгруппа индекса $p$ в $\mathscr{Q}(r, s)$.

В работе [41] предпринято систематическое исследование этих групп и, в частности, найдены их связи с градуированными $\mathbb{Z}_{p}$-алгебрами Ли. Следующий результат описывает ряд свойств этих групп, найденных в [41].

Теорема 4.30. а) Группы $\mathscr{Q}^{1}(r, s)$ являются наследственно едва бесконечными группами конечной ширины. При этом они не являются линейными топологическими группами ни над каким про-р-кольцом.

b) Группь $\left\{\mathscr{Q}^{1}(1, s)\right\}_{s=1}^{\infty}$ попарно несоизмеримъ.

c) $\operatorname{hdim} \mathscr{Q}(r, s)=3 / p^{s}$.

Вычисление хаусдорфовой размерности в пункте с) добавляет ранее неизвестную точку $3 / p$ в хаусдорфовом спектре группы $\mathscr{J}\left(\mathbb{Z}_{p}\right)$. Кроме того, в [41] была найдена еще одна ранее неизвестная точка спектра $3 /(2 p)$. В настоящее время неясной частью хаусдорфова спектра группы $\mathscr{J}\left(\mathbb{Z}_{p}\right)$ при нечетном $p$ остается интервал $(1 / p, 2 / p)$.

4.7. Коммутаторы и степени. Как читатель, возможно, уже успел заметить, структура коммутаторов элементов и взаимных коммутантов подгрупп в $\mathscr{J}(\mathbf{k})$ играет важную роль в изучении самой этой группы. Это обстоятельство было отмечено еще в работе Дженнигса [59], где был вычислен начальный член коммутатора двух элементов в $\mathscr{J}(\mathbf{k})$, см. лемму 2.3. В этой же работе были установлены общие включения (6) для коммутантов. Легко видеть, что в конечной характеристике первый член коммутатора двух элементов (лемма 2.3, b) может обращаться в 0 и для вычислений необходимо знать следующий (ненулевой) член коммутатора. Это важное вычисление было проведено в малодоступной диссертации Йорка 1990 г. Из-за относительной громоздкости этой формулы мы ее не приводим, отсылая интересующихся, например, к [66].

Взаимный коммутант двух замкнутых подгрупп некоторой топологической группы, вообще говоря, не обязан быть замкнутой подгруппой. Это подчеркивает ценность следующего утверждения (см. [25]).

ПреДЛОЖЕНИЕ 4.31. Пусть $\mathbb{F}_{q}$ - конечное поле характеристики $p>2$. Тогда для любых натуральных $n$ и $m$ справедливо равенство

$$
\left[\mathscr{J}^{n}\left(\mathbb{F}_{q}\right), \mathscr{J}^{m}\left(\mathbb{F}_{q}\right)\right]=\overline{\left[\mathscr{J}^{n}\left(\mathbb{F}_{q}\right), \mathscr{J}^{m}\left(\mathbb{F}_{q}\right)\right]}=\mathscr{J}^{l}\left(\mathbb{F}_{q}\right),
$$


где $l=n+m$, если $n \neq m \bmod p, u l=n+m+1$, если $n=m \bmod p$.

Это утверждение сразу позволяет вычислить структуру последовательных коммутантов и членов нижнего центрального ряда группы $\mathscr{J}\left(\mathbb{F}_{q}\right)$ в характеристике, отличной от 2. Несложное индуктивное вычисление приводит к следующим равенствам:

$$
\left[\mathscr{J}\left(\mathbb{F}_{q}\right)\right]_{n}=\mathscr{J}^{2^{n+1}-1}\left(\mathbb{F}_{q}\right) \quad \text { и } \quad \gamma_{n}\left(\mathscr{J}\left(\mathbb{F}_{q}\right)\right)=\mathscr{J}^{n+1+[(n-2) /(p-1)]}\left(\mathbb{F}_{q}\right) .
$$

Таким образом, при $p>2$ и всех натуральных $n$ справедливо неравенство

$$
\left|\gamma_{n+1}\left(\mathscr{J}\left(\mathbb{F}_{q}\right)\right) / \gamma_{n}\left(\mathscr{J}\left(\mathbb{F}_{q}\right)\right)\right| \leqslant q^{2},
$$

т. е. $\mathscr{J}\left(\mathbb{F}_{q}\right)$ является про-р-группой конечной ширинъ. Мы это понятие не обсуждаем, отсылая к монографии [64], посвященной систематическому изучению про- $p$-групп конечной ширины.

Как обычно, характеристика 2 существенно сложнее и требует гораздо более аккуратных вычислений. Нижний центральный ряд группы $\mathscr{J}\left(\mathbb{Z}_{2}\right)$ был вычислен Лидхэм-Грином и Маккэем [71], его описание достаточно громоздко, и мы его не приводим (см. [71] или обзор [25]). Заметим только, что $\mathscr{J}\left(\mathbb{Z}_{2}\right)$ также имеет конечную ширину.

Вычисление ряда последовательных коммутантов группы $\mathscr{J}\left(\mathbb{F}_{2^{d}}\right)$ сложнее, чем вычисление нижнего центрального ряда. Первый результат в этом направлении был получен Хегедюсом [54] и дает хорошее асимптотическое поведение индекса подгрупп $\left[\mathscr{J}\left(\mathbb{F}_{2^{d}}\right)\right]_{n}$ для больших $n$.

Теорема 4.32. Для $n$-го коммутанта группь $\mathscr{J}\left(\mathbb{F}_{2^{d}}\right)$ имеет место включение

$$
\left[\mathscr{J}\left(\mathbb{F}_{2^{d}}\right)\right]_{n} \supseteq \mathscr{J}^{\left[4 K^{n}\right]}\left(\mathbb{F}_{2^{d}}\right),
$$

где $K=(1+\sqrt{17}) / 2$. Таким образом, индекс $\left[\mathscr{J}\left(\mathbb{F}_{2^{d}}\right)\right]_{n}$ не превосходит $2^{4 d K^{n}}$.

Как отмечено в [54], полученная оценка асимптотически точна, т. е.

$$
\lim _{n \rightarrow \infty}\left(\log _{2}\left|\mathscr{J}\left(\mathbb{F}_{2^{d}}\right):\left[\mathscr{J}\left(\mathbb{F}_{2^{d}}\right)\right]_{n}\right|\right)^{1 / n}=K .
$$

Точное вычисление ряда коммутантов группы $\mathscr{J}\left(\mathbb{Z}_{2}\right)$ было получено совсем недавно Богатыми [14]. Как и для нижнего центрального ряда, из-за громоздкости формулировок мы не приводим этот результат, отсылая читателя к [14].

ЗАмЕчАНИЕ 4.33. В начале 1990-х годов возникла дискуссия о возможной p-адической аналитичности групп $\mathscr{J}\left(\mathbb{Z}_{p}\right)$, см. [97]. Позднее выяснилось, что группы $\mathscr{J}\left(\mathbb{Z}_{p}\right)$ не несут никакой $р$-адически аналитической структуры при всех $p$. Так, например, из теорем 4.19 и 4.32 следует, что $\mathscr{J}\left(\mathbb{F}_{2^{d}}\right)$ не является аналитической группой ни над каким про-2-кольцом, строгое обоснование этого факта дано в [98].

В п. 3.2 было рассмотрено поведение степеней элементов группы $\mathscr{J}(\mathbf{k})$ по модулю фиксированной подгруппы $\mathscr{J}^{n}(\mathbf{k})$. В определенном смысле двойственной проблемой является поведение степеней самих подгрупп $\mathscr{J}^{n}(\mathbf{k})$ в зависимости от $n$. В случае конечного поля нечетной характеристики этот вопрос был решен Лидхэм-Грином и Маккэем [71]. 
Теорема 4.34. Для конечного поля нечетной характеристики р имеет место равенство

$$
\left(\mathscr{J}^{n}\left(\mathbb{F}_{q}\right)\right)^{(p)}=\overline{\left(\mathscr{J}^{n}\left(\mathbb{F}_{q}\right)\right)^{(p)}}=\mathscr{J}^{n p+r}\left(\mathbb{F}_{q}\right),
$$

где $r$ - остаток от деления $n$ на $p$.

Как уже неоднократно отмечалось выше, характеристика 2 и в этом вопросе заметно сложнее, чем случай нечетной характеристики. Автору не известны результаты в этом направлении, кроме общего включения $\left(\mathscr{J}^{n}(\mathbf{k})\right)^{(2)} \subseteq$ $\mathscr{J}^{2 n+r}(\mathbf{k})$, где $\mathbf{k}$ - кольцо характеристики 2 , а $r=\{0,1\}$ - остаток от деления $n$ на 2.

Сложность проблемы в характеристике 2 проясняет следующее простое наблюдение. Легко показать, что $\left(\mathscr{J}\left(\mathbb{Z}_{2}\right)\right)^{(2)} \subseteq \mathscr{J}^{3}\left(\mathbb{Z}_{2}\right)$, но при этом $\left(\mathscr{J}\left(\mathbb{Z}_{2}\right)\right)^{(2)} \nsubseteq$ $\mathscr{J}^{4}\left(\mathbb{Z}_{2}\right)$. Более того, оказывается, что для любого элемента $f(x) \in \mathscr{J}\left(\mathbb{Z}_{2}\right)$ имеет место совпадение коэффициентов $\alpha_{4}\left(f(x)^{(2)}\right)=\alpha_{6}\left(f(x)^{(2)}\right)$. Отсюда легко следует совпадение коэффициентов $\alpha_{4}(g(x))=\alpha_{6}(g(x))$ для любого элемента $g(x) \in \overline{\left(\mathscr{J}\left(\mathbb{Z}_{2}\right)\right)^{(2)}}$.

Это наблюдение показывает, что $\overline{\left(\mathscr{J}\left(\mathbb{Z}_{2}\right)\right)^{(2)}}$ - собственная нормальная подгруппа в $\mathscr{J}^{3}\left(\mathbb{Z}_{2}\right)$, не лежащая в $\mathscr{J}^{4}\left(\mathbb{Z}_{2}\right)$ и не содержащая $\mathscr{J}^{6}\left(\mathbb{Z}_{2}\right)$. Таким образом, теорема 4.34 в характеристике 2 несправедлива.

Интересным дополнением к теореме 4.34 может служить результат Китинга [62] о поведении степеней элементов группы $\mathscr{J}(\mathbf{k})$, где $\mathbf{k}$ - кольцо с единицей характеристики $p>0$. Точная формулировка этого результата требует достаточно громоздких и специальных определений, поэтому мы его не приводим, отсылая читателя к цитируемой работе.

В связи с общей проблемой оценки числа классов сопряженных в конечной p-группе были проведены вычисления этого числа в группах $\mathscr{J}_{n}\left(\mathbb{Z}_{p}\right)$. Так, в диссертации Йорка число классов сопряженных было вычислено для $n \leqslant$ $p+1$. Следующее продвижение, включающее вычисления Йорка, было сделано Палфи [87]. В этой работе вычислено число классов, сопряженных в группах $\mathscr{J}_{n}\left(\mathbb{Z}_{p}\right)$ при $n \leqslant 3 p+1$. Эти вычисления показывают, что группы $\mathscr{J}_{n}\left(\mathbb{Z}_{p}\right)$ имеют аномально малое число классов сопряженных среди $p$-групп данного порядка и в этом смысле они максимально далеки от коммутативных $p$-групп. Насколько известно автору, точных вычислений числа классов сопряженных при $n$, больших $3 p+1$, пока нет.

\section{5. Нулевая характеристика}

5.1. Некоторые общие свойства. Всюду в разделе 5 основное кольцо $\mathbf{k}$ предполагается имеющим нулевую характеристику. В этом случае группы $\mathscr{J}(\mathbf{k})$ имеют структуру, совершенно отличную от структуры групп, рассмотренных в предыдущих пунктах. Наиболее интересными с топологической точки зрения здесь являются группы $\mathscr{J}(\mathbb{Z})$ и $\mathscr{J}(\mathbb{R})$. Сначала напомним несколько общих определений. Будем говорить, что $G$ является группой с однозначным извлечением корней (ОИК-группой), если уравнение

$$
x^{n}=g
$$


допускает не более одного решения для любого $g \in G$ и любого натурального $n$. Будем говорить, что $G$ является алгебраически полной (иногда говорят делимой) группой, если уравнение (29) имеет решения для всех $g \neq e$ и всех натуральных $n$.

Пусть $G$ - некоторая ОИК-группа; скажем, что группа $\widehat{G}$ является пополнением группы $G$, если

1) $G \subseteq \widehat{G}$

2) $\widehat{G}$ алгебраически полна;

3) $\widehat{G}$ - минимальная группа, обладающая свойствами 1) и 2).

Последнее означает, что если $G^{\prime}$ - полная группа, удовлетворяющая включениям $G \subseteq G^{\prime} \subseteq \widehat{G}$, то $G^{\prime}=\widehat{G}$.

ПримеР 5.1. Группа гомеоморфизмов $G=\mathrm{Homeo}_{+}[0,1]$, уже встречавшаяся выше в связи с проблемой сжимаемости, алгебраически полна, но не является ОИК-группой.

ПримеР 5.2. Любая нильпотентная группа без кручения $G$ является ОИКгруппой. Согласно классической теореме Мальцева [75] существует пополнение такой группы. Это пополнение, часто называемое пополнением по Мальцеву, единственно с точностью до изоморфизма. Более того, оно функториально, в частности, любой автоморфизм $G$ однозначно продолжается до автоморфизма пополнения. Согласно другой известной теореме Мальцева [74], если $G$ конечно порождена, то она вкладывается в односвязную нильпотентную группу Ли как равномерная решетка.

Некоторые свойства группы $\mathscr{J}(\mathbf{k})$ в случае кольца нулевой характеристики уже встречались выше в предложении 2.15. Дополним их следующим утверждением.

ПредЛОжЕНИЕ 5.3. Группа $\mathscr{J}(\mathbb{Z})$ является ОИК-группой. Группъ $\mathscr{J}(\mathbb{Q})$ и $\mathscr{J}(\mathbb{R})$ алгебраччески полны. Более того, $\mathscr{J}(\mathbb{Q})$ - пополнение группь $\mathscr{J}(\mathbb{Z})$ и это пополнение определено однозначно с точностью до автоморфизма.

Как и в случае простого поля характеристики $p$, группа $\mathscr{J}(\mathbb{Z})$ конечно порождена как топологическая группа. Следующий результат [2] описывает возможные канонические наборы топологических образующих этой группы.

Теорема 5.4. Следующие четыре элемента:

$$
\begin{array}{ll}
f_{1}(x)=x(1+x)+\mathscr{O}\left(x^{3}\right), & f_{2}(x)=x\left(1+x^{2}\right)+\mathscr{O}\left(x^{4}\right), \\
f_{4}(x)=x\left(1+x^{4}\right)+\mathscr{O}\left(x^{6}\right), & f_{6}(x)=x\left(1+x^{6}\right)+\mathscr{O}\left(x^{8}\right)
\end{array}
$$

топологически порождают $\mathscr{J}(\mathbb{Z})$ при любом выборе остаточных членов $\mathscr{O}\left(x^{i}\right)$, $i=3,4,6,8$. Топологическая группа $\mathscr{J}(\mathbb{Z})$ не может быть задана менее чем четыръмя образующими.

ЗАмечАнИЕ 5.5. Теорему 4.5 несложно вывести из теоремы 5.4, используя отображение $\mathscr{J}(\mathbb{Z}) \rightarrow \mathscr{J}\left(\mathbb{Z}_{p}\right)$, индуцированное приведением $\bmod p$ базисного кольца. 
Доказательство того, что 4 указанных элемента топологически порождают $\mathscr{J}(\mathbb{Z})$, может быть проведено индукцией с шагом 4. Более деликатной проблемой является доказательство минимальности этой системы образующих. Минимальность следует из вычисления одномерных непрерывных гомологий группы $\mathscr{J}(\mathbb{Z})$, этот результат представляет независимый интерес, детали см. в [2]. Пусть $G$ - топологическая группа, положим

$$
H_{1}(G ; \mathbb{Z})=G / \overline{[G, G]},
$$

где черта, как обычно, обозначает замыкание.

ТеОРема 5.6. Имеет место изоморфизм

$$
H_{1}(\mathscr{J}(\mathbb{Z}) ; \mathbb{Z}) \simeq \mathbb{Z} \oplus \mathbb{Z} \oplus \mathbb{Z}_{2} \oplus \mathbb{Z}_{2} .
$$

В случае конечной характеристики группа $\mathscr{J}\left(\mathbb{Z}_{p}\right)$ обладает мерой Хаара $\mathbf{m}(p)$ и, как мы видели (теорема 4.6), $n$ случайных элементов этой группы порождают свободную подгруппу. В частности, любой случайный выбор остаточных членов в системе образующих теоремы 4.5 приводит к свободной подгруппе, порожденной этими образующими. Хотя группа $\mathscr{J}(\mathbb{Z})$ не имеет меры Хаара, аналогичный эффект имеет место и для этой группы.

Измерить случайность выбора нескольких элементов группы $\mathscr{J}(\mathbb{Z})$ можно не в смысле меры, а в смысле плотности, определенной на множестве канонических координат на группе $\mathscr{J}(\mathbb{Z})$. Понятие плотности навеяно соответствующими идеями из теории целых аналитических функций (см. [73]). Дадим основные определения, отсылая за деталями к [2].

ОПРЕДЕлЕНИЕ 5.7. Пусть $M$ - бесконечное нигде не плотное подмножество в $\mathbb{R}^{n}$ и $\tau$ - положительное вещественное число. Если величина

$$
p_{\tau}(M)=\limsup _{t \rightarrow \infty} \frac{\#\left(M \cap B_{\infty}(t)\right)}{(2 t)^{\tau}}
$$

положительна и конечна, то назовем ее плотностъю порядка $\tau$ множества $M$. Здесь $B_{\infty}(t)$ - шар радиуса $t$ в $l_{\infty}$-норме на $\mathbb{R}^{n}$, а \# $X$ - мощность множества $X$.

Рассмотрим естественную проекцию $\pi_{n}: \mathbb{Z}^{\infty} \rightarrow \mathbb{Z}^{n} \subset \mathbb{R}^{n}$ на первые $n$ координат.

ОПРЕДЕЛЕНИЕ 5.8. Пусть $M-$ подмножество в $\mathbb{Z}^{\infty}$. Назовем плотностъю коразмерности $k$ множества $M$ величину

$$
p^{k}(M)=\limsup _{n \rightarrow \infty} p_{n-k}\left(\pi_{n}(M)\right) .
$$

Наконец, пусть $N \subset(\mathscr{J}(\mathbb{Z}))^{s} \simeq\left(\mathbb{Z}^{\infty}\right)^{s}$ - аффинное подмножество коразмерности $k$ и $M \subset N$ - подмножество. Скажем, что почти любой элемент $N$ содержится в $M$, если $p^{k}(M)=1$.

Произвольный выбор остаточных членов топологических образующих $f_{1}, f_{2}$, $f_{4}, f_{6}$ группы $\mathscr{J}(\mathbb{Z})$ задает аффинную подрешетку $N \subset(\mathscr{J}(\mathbb{Z}))^{4}$. Из теоремы 5.4 заключаем, что $\operatorname{codim} N=13$. Пусть $M \subset N$ состоит из всех четверок элементов, порождающих свободную группу ранга 4 в $\mathscr{J}(\mathbb{Z})$. Массивность подмножества $M$ утверждает следующее предложение [2]. 
ПРЕДЛОЖЕНИЕ 5.9. Имеет место равенство $p^{13}(M)=1$.

Аналогично следствию 4.7 получаем следующее утверждение.

СлеДСТвиЕ 5.10. Образующие $f_{1}, f_{2}, f_{4}, f_{6}$ теоремы 5.4 порождают в $\mathscr{J}(\mathbb{Z})$ свободную подгруппу ранга 4 при почти любом в смысле плотности выборе остаточных членов.

5.2. Бесконечномерные группы Ли. В этом пункте нам понадобятся некоторые основные факты дифференциального исчисления в локально выпуклых топологических (ЛВТ) векторных пространствах. В частности, мы будем использовать понятие $C^{\infty}$-отображения и аналитического отображения таких пространств, детали можно найти в замечательных лекциях Милнора [81]. Напомним несколько общих определений. Пусть $X$ и $Y$ - два линейных ЛВТ пространства, $U \subset X$ - открытое множество и $f: U \rightarrow Y$ - некоторое отображение. Производная по Гато определяется как предел

$$
f^{\prime}(x ; v)=\lim _{t \rightarrow 0} \frac{f(x+t v)-f(x)}{t},
$$

если, разумеется, он существует.

Будем говорить, что $f$ класса $C^{1}(U)$, если предел (30) существует для всех $v \in X, x \in U$ и отображение $f^{\prime}(x ; v)$ непрерывно как функция двух переменных.

Скажем, что $f$ класса $C^{2}(U)$, если $f \in C^{1}(U)$ и для любого фиксированного $v_{1} \in X$ отображение $x \mapsto f^{\prime}\left(x ; v_{1}\right)$, переводящее $U$ в $Y$, снова является отображением класса $C^{1}(U)$, где производная Гато

$$
f^{\prime}\left(x ; v_{1}, v_{2}\right)=\lim _{t \rightarrow 0} \frac{f\left(x+t v_{2} ; v_{1}\right)-f\left(x ; v_{1}\right)}{t}
$$

непрерывна уже по трем переменным $x, v_{1}, v_{2}$.

Продолжая далее по индукции, последовательно определяем высшие классы дифференцируемости отображений $U$ в $Y$. Можно показать, что если $f \in$ $C^{r}(U)$, то производная $f^{r}\left(x ; v_{1}, v_{2}, \ldots, v_{r}\right)$ является симметрической функцией аргументов $v_{1}, v_{2}, \ldots, v_{r}$.

Будем говорить, что $f$ класса $C^{\infty}(U)$, если $f \in C^{r}(U)$ для любого натурального $r$. Возможно также определить понятие аналитического отображсения, детали см. в [81].

Группы Ли, смоделированные на некотором ЛВТ векторном пространстве, определяются обычным образом, детали, опять же, см. в [81]. Для нас основными модельными ЛВТ векторными пространствами будут $\mathbb{R}^{\infty}=\mathbb{R}^{\mathbb{N}}$ и $\mathbb{C}^{\infty}=\mathbb{C}^{\mathbb{N}}$.

ЗАмЕчАниЕ 5.11. Часто рассматриваются группы Ли, смоделированные на некотором банаховом пространстве. В этом случае дифференцируемости можно придать более привычную форму с использованием производной Фреше. Однако класс бесконечномерных групп Ли, смоделированных на ЛВТ векторных пространствах, более богат интересными примерами, чем класс банаховых групп Ли. Так, например, группы диффеоморфизмов принадлежат именно классу ЛВТ групп Ли. Интересующие нас бесконечномерные группы Ли также принадлежат этому классу и не могут быть смоделированы на банаховом 
пространстве. В этой связи отметим, что согласно известному результату Торунчика имеет место гомеоморфизм $\mathbb{R}^{\infty} \simeq l_{2}$. Тем не менее этот гомеоморфизм чисто топологический и никак не связан с линейной структурой пространств. Как ЛВТ линейные пространства $\mathbb{R}^{\infty}$ и $l_{2}$ совершенно различны.

Теорема 5.12. Группа $\mathscr{J}(\mathbb{R})(\mathscr{J}(\mathbb{C}))$ является бесконечномерной аналитической (комплексной аналитической) односвязной группой Ли, смоделированной на ЛВТ векторном пространстве $\mathbb{R}^{\infty}\left(\mathbb{C}^{\infty}\right)$.

Поскольку группа $\mathscr{J}(\mathbb{R})(\mathscr{J}(\mathbb{C}))$ покрывается одной-единственной картой, естественно гомеоморфной $\mathbb{R}^{\infty}\left(\mathbb{C}^{\infty}\right)$, то проблема замены координат не возникает вовсе. Остается проверить аналитичность операции умножения и отображения взятия обратного элемента $f \mapsto f^{-1}$. Это легко делается с использованием формул (4) и (5).

5.3. Алгебра Ли. Чтобы найти алгебру Ли $L(\mathscr{J}(\mathbb{R}))$ группы $\mathscr{J}(\mathbb{R})$, поступим обычным способом, а именно, рассмотрим гладкую кривую

$$
\gamma(t)=f_{t}(x)=x\left(1+\alpha_{1}(t) x+\alpha_{2}(t) x^{2}+\cdots\right), \quad \gamma(0)=x .
$$

Дифференцируемость $\gamma(t)$ по $t$ эквивалентна дифференцируемости функций $\alpha_{i}(t)$ по $t$. Чтобы найти касательный вектор в нуле к этой кривой, интерпретируем функции на $\mathscr{J}(\mathbb{R})$ как элементы локального кольца $h(x) \in \mathbb{R}[[x]]$. Обозначим $V$ искомый касательный вектор. Справедливо равенство

$$
\frac{d h}{d V}=\frac{d}{d t}_{\mid t=0} h(\gamma(t))=\frac{d h}{d x} \frac{d \gamma(t)}{d t}_{\mid t=0}=a(x) \frac{d}{d x}(h(x)),
$$

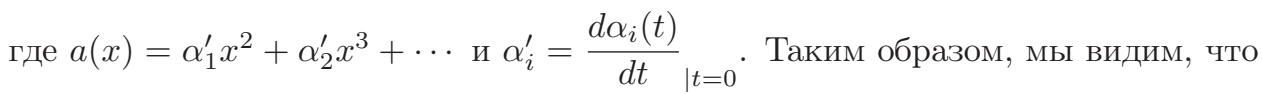
векторы, касательные в точке $x$ (единичный элемент группы $\mathscr{J}(\mathbb{R})$ ), ассоциированы с формальными дифференциальными операторами на прямой $\mathbb{R}$ :

$$
\frac{d \gamma(t)}{d t}_{\mid t=0}=a(x) \frac{d}{d x}
$$

где $a(x)$ принадлежит $\left(x^{2}\right)$ - идеалу, порожденному $x^{2}$ в $\mathbb{R}[[x]]$. Операторы

$$
W_{1}(1)=\left\{a(x) \frac{d}{d x}, a(x) \in\left(x^{2}\right)\right\}
$$

образуют хорошо известную бесконечномерную алгебру Ли, скобка задается формулой

$$
\left[a(x) \frac{d}{d x}, b(x) \frac{d}{d x}\right]=\left(a(x) \frac{d b(x)}{d x}-b(x) \frac{d a(x)}{d x}\right) \frac{d}{d x} .
$$

Это топологическая алгебра Ли, возникающая топология идентична с топологией на $\mathbb{R}^{\infty}$. В качестве топологической базы обычно берется следующая последовательность векторов:

$$
e_{k}=x^{k+1} \frac{d}{d x}, \quad k=1,2, \ldots ; \quad\left[e_{n}, e_{m}\right]=(m-n) e_{n+m}, \quad n, m \geqslant 1 .
$$


Алгебра $W_{1}(1)$ является нильпотентной частью известной алгебры Витта $W(1)$, состоящей из всех формальных векторных полей на вещественной прямой. Топологическая база $W(1)$ может быть задана теми же формулами (31), где $k, n$ и $m$ пробегают все целые числа $\mathbb{Z}$. Алгебру $W_{1}(1)$, заданную в $(31)$, можно рассматривать над любым кольцом $\mathbf{k}$ нулевой характеристики. Во избежание недоразумений в этом случае будем писать $W_{1}^{\mathbf{k}}(1)$.

Если $\mathbf{k}$ - поле нулевой характеристики, то на алгебре $W_{1}^{\mathbf{k}}(1)$ определены гомотетии, действующие на векторы канонического базиса по правилу

$$
\Delta_{s}\left(e_{n}\right)=s^{n} e_{n}, \quad s \in \mathbf{k}, \quad n=1,2, \ldots .
$$

Несложно проверить, что при всех $s \neq 0$ гомотетия (32) будет автоморфизмом алгебры Ли $W_{1}^{\mathbf{k}}(1)$.

5.4. Экспоненциальное отображение. Как обычно, экспоненциальное отображение каждому вектору $V \in W_{1}(1)$ ставит в соответствие точку $\gamma(1) \in$ $\mathscr{J}(\mathbb{R})$, где $\gamma(t)$ - однопараметрическая подгруппа в $\mathscr{J}(\mathbb{R})$, удовлетворяющая условию $\frac{d \gamma(t)}{d t}=V$. Аналогичное определение имеет смысл и в комплексной ситуации, причем $t$ также считается комплексным параметром.

ПрЕДЛОЖЕНИЕ 5.13. Пусть $\mathbf{k}=\mathbb{R}$ или $\mathbb{C}$. Экспоненциальное отображение $\operatorname{exp:~} W_{1}^{\mathbf{k}}(1) \rightarrow \mathscr{J}(\mathbf{k})$ задается формулой

$$
\exp (V)=\sum_{k=0}^{\infty} \frac{V^{k}(x)}{k !}
$$

где $V^{k}(x)$ - $k$-я итерачия дифберенциального оператора $V=a(x) \frac{d}{d x}$, nримененная $к$ функчии $x$.

ЗАмЕчАНИЕ 5.14. Несложно показать, что формула (33) задает диффеоморфизм $W_{1}^{\mathbf{k}}(1)$ на $\mathscr{J}(\mathbf{k})$, где $\mathbf{k}=\mathbb{R}$ или $\mathbb{C}$. Отметим также, что формула $(33)$ верна для любого кольца $\mathbf{k}$ нулевой характеристики, содержащего поле рациональных чисел $\mathbb{Q}$.

Далее в этом пункте мы всегда считаем, что $\mathbf{k}=\mathbb{R}$ или $\mathbb{C}$. Пусть

$$
V=\left(\sum_{k=1}^{\infty} \alpha_{k}^{\prime} x^{k+1}\right) \frac{d}{d x} \quad \text { и } \quad \exp (V)=x\left(1+\alpha_{1} x+a_{2} x^{2}+\cdots\right) .
$$

Из (33) находим, что $\alpha_{k}=\chi_{k}\left(\alpha_{1}^{\prime}, \alpha_{2}^{\prime}, \ldots, \alpha_{k}^{\prime}\right)$, где $\chi_{k}$ является универсальным многочленом с рациональными коэффициентами, имеющим мультистепень $k$. Параметры $\left(\alpha_{1}^{\prime}, \alpha_{2}^{\prime}, \ldots\right)$ можно рассматривать как мальцевские координаты первого рода (см. [74]) на группе $\mathscr{J}(\mathbb{R})$. Экспоненциальное отображение в форме (33), а также предложение 5.13 были первоначально установлены в работе Дженнингса [59].

Несложно найти однопараметрические подгруппы, отвечающие каноническому базису (31). Обозначим $\gamma_{n}(t)$ однопараметрическую подгруппу, вектор 
скорости которой в $t=0$ есть $x^{n+1} \frac{d}{d x}$. Решение соответствующего обыкновенного дифференциального уравнения приводит к

$$
\gamma_{n}(t)=\frac{x}{\left(1-n t x^{n}\right)^{1 / n}} .
$$

ЗАмЕчание 5.15. Разложение $\gamma_{n}(t)$ в биномиальный ряд по $x$ имеет рациональные коэффициенты. Тем не менее, если $n$ взаимно просто с $p$, то коэффициенты этого ряда оказываются корректно определенными в $\mathbb{Z}_{p}$. Это означает, что при $(n, p)=1$ формула (34) задает однопараметрическую подгруппу в $\mathscr{J}\left(\mathbb{Z}_{p}\right)$. Это те же самые подгруппы, которые были определены в $(22)$.

Оказывается, что экспонента коммутирует с гомотетиями $\Delta_{s}$ и $\delta_{s}$. Это означает, что для любых $V \in W_{1}^{\mathbf{k}}(1)$ и $s \in \mathbf{k}$ имеет место равенство

$$
\delta_{s} \exp (V)=\exp \left(\Delta_{s}(V)\right)
$$

Для нахождения обратного отображения, логарифма, может быть использовано следующее дифференциальное уравнение. Пусть $f(x) \in \mathscr{J}(\mathbf{k})$, тогда вектор $V=v(x) \frac{d}{d x} \in W_{1}^{\mathbf{k}}(1)$ таков, что $\exp (V)=f(x)$ удовлетворяет уравне-
нию

$$
v(f(x))=v(x) \frac{d f(x)}{d x} .
$$

При этом коэффициенты ряда $v(x)=\sum_{k=1}^{\infty} \alpha_{k}^{\prime} x^{k+1}$ однозначно восстанавливаются по коэффициентам ряда $f(x)=x\left(1+\alpha_{1} x+\alpha_{2} x^{2}+\cdots\right)$ так, что $\alpha_{k}^{\prime}=$ $\theta_{k}\left(\alpha_{1}, \alpha_{2}, \ldots, \alpha_{k}\right)$ - универсальные полиномы мультистепени $k$ с рациональными коэффициентами.

Уравнение (35) было впервые введено в 1950-е годы Жаботинским в связи с исследованием аналитичности однопараметрической подгруппы, проходящей через данный элемент $f(x)$, представляющий аналитичную в некоторой окрестности нуля функцию, см. [40], [57] и следующий раздел 6. Заметим, что в этих ранних работах решение $v(x)$ уравнения (35) не связывалось с соответствующей алгеброй Ли, а воспринималось как некоторый формальный ряд, ассоциированный с исходным элементом $f(x)$.

5.5. $\mathscr{J}(\mathbb{Z})$ как подгруппа в $\mathscr{J}(\mathbb{R})$. Группа $\mathscr{J}(\mathbb{Z})$ естественно вкладывается в $\mathscr{J}(\mathbb{R})$ и играет там роль "решетки". Как мы увидим, подгруппа $\mathscr{J}(\mathbb{Z})$, действительно, кокомпактна, но не является дискретной подгруппой. В самом деле, единичный элемент $x \in \mathscr{J}(\mathbb{R})$ не будет изолированной точкой подмножества $\mathscr{J}(\mathbb{Z}) \subset \mathscr{J}(\mathbb{R})$.

Группы $\mathscr{J}(\mathbb{Z})$ и $\mathscr{J}(\mathbb{R})$ не являются ни компактными, ни локально компактными, тем не менее факторпространство

$$
K_{\infty}=\mathscr{J}(\mathbb{R}) / \mathscr{J}(\mathbb{Z})
$$

компактно и обладает рядом замечательных свойств. Пространство $K_{\infty}$ играет роль классифицирующего пространства группы $\mathscr{J}(\mathbb{Z})$. Следующий результат [2] описывает некоторые его свойства. 
ТеОРема 5.16. Пространство $K_{\infty}$ является компактным метрическим топологически однородным пространством, обладающим следующими свойствами.

а) Естественная проекиия $p_{\infty}: \mathscr{J}(\mathbb{R}) \rightarrow K_{\infty}$ является главным расслоением в смысле Гуревича. Она обладает свойством единственности поднятия пути, но не является накрытием.

b) Компакт $K_{\infty}$ является пространством Эйленберга-Маклейна типа $K(\mathscr{J}(\mathbb{Z}), 1)$.

с) Пространство $K_{\infty}$ не является локально полуодносвязным, оно не имеет универсального накрытия и гомотопически не эквивалентно никакому CW-комплексу.

d) $\mathrm{Ha} K_{\infty}$ существует борелевская вероятностная мера $\mathbf{m}$, инвариантная относительно естественного левого действия группь $\mathscr{J}(\mathbb{R})$ на $K_{\infty}$.

е) Существует непрерьвное отображение $P: I^{\mathbb{N}} \rightarrow K_{\infty}$, индуцирующее изоморфизм пространств с мерой $\left(I^{\mathbb{N}},(d x)^{\otimes \mathbb{N}}\right) u\left(K_{\infty}, \mathbf{m}\right)$, где $d x-$ мера Лебега на отрезке $I=[0,1]$.

Введенные в п. 2.3 группы полиномов $\mathscr{J}_{n}(\mathbb{R})$ и $\mathscr{J}_{n}(\mathbb{Z})$ оказываются чрезвычайно полезными при изучении группы $\mathscr{J}(\mathbb{Z})$ и ее классифицирующего пространства $K_{\infty}$. Факторизация односвязной нильпотентной группы Ли $\mathscr{J}_{n}(\mathbb{R})$ по равномерной решетке $\mathscr{J}_{n}(\mathbb{Z})$ приводит к $n$-мерному нильмногообразию

$$
K_{n}=\mathscr{J}_{n}(\mathbb{R}) / \mathscr{J}_{n}(\mathbb{Z}) .
$$

Мера Хаара на $\mathscr{J}_{n}(\mathbb{R})$ опускается на $K_{n}$, что приводит к мере $\mathbf{m}_{n}$ на $K_{n}$, которую мы считаем нормализованной условием единичности объема. Заметим, что мера $\mathbf{m}_{n}$ инвариантна относительно естественного левого действия $\mathscr{J}_{n}(\mathbb{R})$ на $K_{n}$. Для пространства $K_{\infty}$ и меры $\mathbf{m}$ на нем справедливы разложения в обратный спектр (см. [2])

$$
K_{\infty}=\lim _{n} K_{n}, \quad \mathbf{m}={\underset{\lim }{n}}_{\mathbf{m}_{n}} .
$$

Многообразия $K_{n}$ обладают рядом интересных и полезных топологических свойств. Так, например, при четном $n$ многообразия $K_{n}$ имеют естественную симплектическую, а при нечетном - контактную структуру. Эти и другие свойства $K_{n}$ проистекают из соответствующих свойств групп $\mathscr{J}_{n}(\mathbb{R})$. Рассмотрим эти группы подробнее.

5.6. Группы $\mathscr{J}_{n}(\mathbb{R})$ и $\mathscr{J}_{n}(\mathbb{Z})$. Как уже кратко указывалось на с. 12 , элементами группы $\mathscr{J}_{n}(\mathbb{R})$ являются многочлены вида

$$
f(x)=x\left(1+\alpha_{1} x+\alpha_{2} x^{2}+\cdots+\alpha_{n} x^{n}\right),
$$

а операцией - подстановка многочлена в многочлен с последующим приведением $\bmod x^{n+2}$. Группа $\mathscr{J}_{n}(\mathbb{R})$ оказывается нильпотентной филиформной односвязной группой Ли размерности $n$. Филиформность означает, что при данной размерности $n$ эта группа имеет максимально возможную ступень нильпотентности $n-1$. Коэффициенты полиномов $\alpha_{1}, \alpha_{2}, \ldots, \alpha_{n}$ в (38) можно рассматривать как глобальные координаты на $\mathscr{J}_{n}(\mathbb{R})$. 
ЗАмЕчАниЕ 5.17. Эти координаты естественны на $\mathscr{J}_{n}(\mathbb{R})$, но не совпадают с мальцевскими координатами ни первого, ни второго рода.

Алгебра Ли $L_{n}$ группы $\mathscr{J}_{n}(\mathbb{R})$ является очевидной редукцией алгебры $W_{1}(1)$. В базисе $e_{k}, k=1,2, \ldots, n$, соответствующем однопараметрическим подгруппам (редукциям подгрупп (34))

$$
\gamma_{k}^{(n)}(t)=\frac{x}{\left(1-k t x^{k}\right)^{1 / k}} \quad \bmod x^{n+2}, \quad k=1,2, \ldots, n,
$$

группы $\mathscr{J}_{n}(\mathbb{R})$, алгебра $L_{n}$ задается соотношениями (ср. с соотношениями $\left.(31)\right)$

$$
\begin{array}{cc}
{\left[e_{k}, e_{m}\right]=(m-k) e_{k+m},} & 1 \leqslant k, m, \quad k+m \leqslant n \\
{\left[e_{k}, e_{m}\right]=0,} & k+m>n .
\end{array}
$$

Рассмотрим двойственный базис $\left\{\omega_{1}, \omega_{2}, \ldots, \omega_{n}\right\} \in L_{n}^{*}$, реализованный инвариантными справа дифференциальными 1-формами на $\mathscr{J}_{n}(\mathbb{R})$. Дифференциалы форм $\left\{\omega_{i}\right\}_{i=1}^{n}$ легко находятся из (39) по формуле Картана $d \omega_{k}\left(e_{i}, e_{j}\right)=$ $\omega_{k}\left(\left[e_{i}, e_{j}\right]\right)$, что дает

$$
d \omega_{1}=d \omega_{2}=0 ; \quad d \omega_{k}=(k-2) \omega_{1} \wedge \omega_{k-1}+(k-4) \omega_{2} \wedge \omega_{k-2}+\cdots .
$$

Заметим, что упоминавшаяся выше мера Хаара $\mathbf{m}_{n}$ на $\mathscr{J}_{n}(\mathbb{R})$ совпадает с мерой Лебега в естественных координатах $\alpha_{1}, \alpha_{2}, \ldots, \alpha_{n}$ и выражается в базиce $\left\{\omega_{i}\right\}_{i=1}^{n}$ KaK

$$
\mathbf{m}_{n}=\omega_{1} \wedge \omega_{2} \wedge \cdots \wedge \omega_{n} .
$$

Хотя дифференциальные формы $\left\{\omega_{i}\right\}_{i=1}^{n}$ лишь правоинвариантны, форма объема $\mathbf{m}_{n}$ уже оказывается биинвариантной.

Анализ формул (40) дает следующее предложение.

ПРЕДЛОЖЕНИЕ 5.18. 1) Если $n=2 k+1, k \geqslant 1$, mо группа Ли $\mathscr{J}_{n}(\mathbb{R})$ uмеeт правоинвариантную контактную структуру, заданную формой $\omega_{n}$.

2) Если $n=2 k$, то группа Ли $\mathscr{J}_{n}(\mathbb{R})$ имеет правоинвариантную симплектическую структуру, заданную 2-формой

$$
\Omega_{2 k}=(2 k-1) \omega_{1} \wedge \omega_{2 k}+(2 k-3) \omega_{2} \wedge \omega_{2 k-1}+\cdots+\omega_{k} \wedge \omega_{k+1} .
$$

Проектируя формы $\omega_{n}$ и $\Omega_{n}$ на многообразие $K_{n}$, мы получим контактную структуру при нечетном $n$ и, соответственно, симплектическую при $n$ четном. Для полученных таким образом структур на $K_{n}$ сохраним те же обозначения: $\omega_{n}$ для контактной и $\Omega_{n}$ для симплектической. Формы $\omega_{n}$ и $\Omega_{n}$ определены на $K_{n}$ при всех $n$ и при этом максимально невырождены в соответствующем смысле. Их контактность (симплектичность) - лишь проявление нечетности (четности) размерности.

Формы $\Omega_{n}$ на $K_{n}$ и $\omega_{n+1}$ на $K_{n+1}$ имеют простую геометрическую связь. Несложно увидеть, что проекция $p_{n}^{n+1}: K_{n+1} \rightarrow K_{n}$ будет главным $S^{1}$-расслоением. При этом $\omega_{n+1}$ является формой связности этого расслоения, а $\Omega_{n}-$ ее форма кривизны: $d \omega_{n+1}=\left(p_{n}^{n+1}\right)^{*}\left(\Omega_{n}\right)$. 


\section{7. Симплектические многообразия $\left(K_{2 n}, \Omega_{2 n}\right)$ и произведения} Масси. Среди многих интересных свойств симплектических многообразий $\left(K_{2 n}, \Omega_{2 n}\right)$ кратко остановимся на следующем. В вещественных когомологиях этих многообразий имеется большой запас нетривиальных произведений Масси, причем вес (длина) этих произведений может быть достаточно большим. Чтобы не перегружать текст, за точными определениями и основными свойствами произведений Масси отошлем читателя к классическим работам [69] и [76], см. также [7], где излагается подход, использующий обобщенное уравнение Маурера-Картана в матричных алгебрах.

Замечательным свойством многообразий $K_{2 n}$ является то, что сам симплектический класс когомологий $\left[\Omega_{2 n}\right]$ разлагается в $2 n$-местное произведение Масси и не может быть выражен в виде некоторой линейной комбинации произведений Масси строго меньшего, чем $2 n$, числа сомножителей.

ПРЕДЛОЖеНИЕ 5.19. Симплектический класс $\left[\Omega_{2 n}\right] \in H^{2}\left(K_{2 n}, \mathbb{R}\right)$ разлагается в 2 -местное матричное произведение Масси веса строго $2 n$.

Это предложение было доказано в [7], где было явно указано такое представление для всех $n$. Представление $\left[\Omega_{2 n}\right]$ в виде произведения Масси, естественно, не единственно, и можно найти даже ординарные (нематричные) произведения, представляющие этот класс. Тем не менее в любом представлении симплектического класса $\left[\Omega_{2 n}\right]$ в виде произведений Масси не может быть меньше $2 n$ сомножителей, детали см. в [7]. Отметим, что поскольку $H^{1}\left(K_{2 n}, \mathbb{R}\right)$ порождено, как векторное пространство, двумя элементами $\left[\omega_{1}\right]$ и $\left[\omega_{2}\right]$, то любые произведения Масси, лежащие в $H^{2}\left(K_{2 n}, \mathbb{R}\right)$, выражаются лишь через $\left[\omega_{1}\right]$ и $\left[\omega_{2}\right]$.

Интерес к произведениям Масси в симплектических многообразиях возник более 20 лет назад и был вызван популярным в то время вопросом - насколько сильно класс компактных симплектических многообразий отличается от класса компактных кэлеровых многообразий?

Напомним, что нетривиальные произведения Масси дают одно из препятствий к формальности. С другой стороны, согласно известной теореме, принадлежащей Делиню, Гриффитсу, Моргану и Сулливану [32], кэлеровы многообразия гомотопически формальны, а следовательно, все высшие произведения Масси в таких многообразиях должны быть тривиальны. Таким образом, наличие в компактном симплектическом многообразии нетривиальных произведений Масси высокого веса позволяет измерить “степень отличия" этого многообразия от кэлерова.

Первые симплектические многообразия с нетривиальными (трехместными) произведениями Масси были найдены Грэем с соавторами в [46], см. также [47]. В [46] было показано, что многообразие $K_{4}$, а также аналогичные нильмногообразия, соответствующие выбору в $\mathscr{J}_{4}(\mathbb{R})$ решеток, отличных от $\mathscr{J}_{4}(\mathbb{Z})$, имеют симплектическую структуру, но не имеют комплексной структуры, а следовательно, и кэлеровой. Здесь препятствием к существованию комплексной структуры является наличие нетривиальных произведений Масси. В качестве наиболее простого такого произведения можно взять $\left\langle\left[\omega_{2}\right],\left[\omega_{1}\right],\left[\omega_{2}\right]\right\rangle \in H^{2}\left(K_{4}, \mathbb{R}\right)$ (это же произведение можно взять при любом другом выборе решетки в $\left.\mathscr{J}_{4}(\mathbb{R})\right)$. Тот факт, что произведения Масси являются препятствием даже к существованию комплексной структуры, - феномен теории комплексных поверхностей. 
Действительно, в рассматриваемом случае первое число Бетти $b_{1}\left(K_{4}\right)$ равно 2. Согласно классическому результату Кодаиры [67], если на четырехмерном многообразии с четным первым числом Бетти существовала бы комплексная структура, то она являлась бы деформацией структуры комплексного алгебраического многообразия. Таким образом, наличие в рассматриваемом примере комплексной структуры было бы эквивалентно наличию некоторой кэлеровой структуры.

На многообразиях $K_{2 n}, n \geqslant 2$, нет комплексных структур, происходящих из правоинвариантных комплексных структур на соответствующих группах $\mathscr{J}_{2 n}(\mathbb{R})$. Это следствие филиформности этих групп Ли [52]. Похоже, что, как и $K_{4}$, многообразия $K_{2 n}, n \geqslant 3$, вообще не могут обладать никакой комплексной структурой, однако точные утверждения по этому поводу автору не известны.

ЗАмЕчАНИЕ 5.20. а) Интересный пример представляет собой многообразие $K_{3} \times S^{1}$, известное в литературе как многообразие Кодаиръ-Тёрстона. Оно имеет симплектическую и согласованную с ней комплексную структуры. Подсчет первого числа Бетти показывает, что оно равно 3 и, следовательно, $K_{3} \times S^{1}$ не обладает никакой кэлеровой структурой.

b) При исследовании произведений Масси в симплектических многообразиях в [6] первоначально были введены многообразия, аналогичные $K_{n}$ и непосредственно опирающиеся на структуру алгебры Ли группы $\mathscr{J}_{n}(\mathbb{R})$. Затем в [21] было предложено использованное выше представление (37). Такое представление, в действительности, более естественно.

Все симплектические многообразия с нетривиальными произведениями Масси, обсуждавшиеся выше, являются неодносвязными. С помощью этих примеров можно построить и односвязные симплектические многообразия, содержащие нетривиальные произведения Масси любого наперед заданного веса. Однако этот интересный сюжет симплектической топологии уже далеко уходит от основного направления настоящей статьи и мы лишь отошлем интересующихся к [5], [6] и [8], где такие многообразия были впервые построены.

5.8. Вещественные когомологии группы $\mathscr{J}(\mathbb{Z})$. Хорошо известно, что выбор подходящего комплекса коцепей (цепей) в когомологиях (гомологиях) конечно порожденной группы позволяет наиболее полно отразить те или иные ее свойства. Для топологических групп наиболее подходящими будут когомологии, учитывающие еще и топологические свойства группы. Поскольку топология на группе $\mathscr{J}(\mathbb{Z})$ вводится как обратный предел топологий на группах $\mathscr{J}_{n}(\mathbb{Z})$, то наиболее естественным является рассмотрение когомологий, которые перестановочны с взятием обратного предела, т. е. непрерывны. В категории компактных групп эту проблему успешно решают когомологии с непрерывными носителями [56], [96]. Рассматриваемая группа $\mathscr{J}(\mathbb{Z})$ некомпактна и даже не локально компактна. Классическая конструкция коцепей с непрерывными носителями оказывается недостаточной, и мы рассматриваем когомологии с равномерно постоянными носителями. Кратко изложим эту конструкцию, детали см. в [2].

Пусть $G$ - топологическая группа и $F$ - топологический $\mathbb{Z}$-модуль. Будем говорить, что $F$ - равномерно локалъно постоянный $G$-бимодуль, если имеются 
такие непрерывные отображения

$$
F \times G \rightarrow F \quad \text { и } \quad G \times F \rightarrow F,
$$

являющиеся автоморфизмами $F$ при фиксированном $g \in G$, что для некоторой окрестности $V \subset G$ единичного элемента $e \in G$ включение $g_{1}^{-1} g_{2} \in V$ влечет равенства $u g_{1}=u g_{2}$ и $g_{1} u=g_{2} u$ для всех элементов $u \in F$.

Отображение $f: G^{q} \rightarrow F$ будем называеть равномерно локалъно постоянной $q$-коцепью с коэффициентами в $F$, если существует окрестность $U_{f}$ единицы $e \in G$ такая, что как только $g_{i}^{-1} h_{i} \in U_{f}, i=1,2, \ldots, q$, то $f\left(g_{1}, g_{2}, \ldots, g_{q}\right)=$ $f\left(h_{1}, h_{2}, \ldots, h_{q}\right)$. В рассматриваемом далее приложении правое и левое действия будут тривиальны, т. е. для всех $g \in G$ и $u \in F$ имеют место равенства $g u=u=u g$, поэтому можно считать, что в определениях коцепей действие группы $G$ отсутствует. На множестве $C^{q}(G ; F)$ всех равномерно локально постоянных коцепей размерности $q$ с коэффициентами в $F$ естественным образом определяется операция сложения:

$$
\left(f_{1}+f_{2}\right)\left(g_{1}, g_{2}, \ldots, g_{q}\right)=f_{1}\left(g_{1}, g_{2}, \ldots, g_{q}\right)+f_{2}\left(g_{1}, g_{2}, \ldots, g_{q}\right),
$$

превращающая $C^{q}(G ; F)$ в абелеву группу.

Оператор кограницы $\delta: C^{q}(G ; F) \rightarrow C^{q+1}(G ; F)$ задается стандартной формулой

$$
\begin{aligned}
(\delta f) & \left(g_{1}, g_{2}, \ldots, g_{q}, g_{q+1}\right)=g_{1} f\left(g_{2}, \ldots, g_{q}, g_{q+1}\right) \\
& +\sum_{i=1}^{q}(-1)^{i} f\left(g_{1}, \ldots, g_{i} g_{i+1}, \ldots, g_{q+1}\right)+(-1)^{q+1} f\left(g_{1}, \ldots, g_{q}\right) g_{q+1} .
\end{aligned}
$$

Поскольку правое и левое действия группы $G$ являются равномерно локально тривиальными, то непосредственно проверяется, что коцепь $\delta f$ является равномерно локально постоянной. Равенство $\delta^{2}=0$ чисто алгебраическое и справедливо на множестве всех коцепей. Таким образом, корректно определены когомологии, которые мы будем обозначать $\widetilde{H}^{*}(G ; F)$.

Легко видеть, что всякая равномерно локально постоянная коцепь является непрерывной коцепью, поэтому определен естественный гомоморфизм

$$
\widetilde{H}^{*}(G ; F) \rightarrow \bar{H}^{*}(G ; F)
$$

в непрерывные когомологии $G$. Если группа $G$ дискретна, то всякая коцепь является равномерно локально постоянной, поэтому для всякой дискретной группы $G$, например группы $J_{n}(\mathbb{Z})$, введенные когомологии естественным образом изоморфны стандартным когомологиям абстрактной группы $G$.

Если топологическая группа $G$ компактна, а модуль коэффициентов $F$ дискретен, то всякая непрерывная коцепь является равномерно локально тривиальной. В этом случае введенные когомологии естественным образом изоморфны непрерывным когомологиям группы $G$ [56]. В этом смысле можно говорить, что введенные группы когомологий являются естественным обобщением непрерывных когомологий проконечных групп на случай общих нульмерных групп.

Следующее предложение [2] обосновывает спектральную непрерывность введенных когомологий. 
ПредлоЖениЕ 5.21. Если топологическая группа $G$ является пределом обратной последовательности групп $\left\{G_{i}, h_{i}^{i+1}\right\}_{i=1}^{\infty}$ с сюргективными гомоморфизмами $h_{i}^{i+1}: G_{i+1} \rightarrow G_{i}$, то

$$
\widetilde{H}^{q}(G ; F)=\underset{i}{\lim }\left\{\widetilde{H}^{q}\left(G_{i} ; F\right),\left(h_{i}^{i+1}\right)^{*}\right\} .
$$

Стандартные конструкции гомотопической топологии позволяют связать когомологии некоторой дискретной группы $G$ с сингулярными когомологиями ее классифицирующего пространства $K(G, 1)$, которое можно выбрать симплициальным полиэдром (в слабой топологии). Теорема 5.16 показывает, что $K_{\infty}$ играет роль классифицирующего пространства группы $\mathscr{J}(\mathbb{Z})$. И хотя $K_{\infty}$ гомотопически не эквивалентен никакому $C W$-комплексу, изоморфизм когомологий также справедлив в этом случае при подходящем выборе теории когомологий. Обозначим $\widehat{H}^{*}(X ; F)$ когомологии Александера-Чеха [100] топологического пространства $X$ в локальной системе коэффициентов $F$. Доказательство следующего утверждения см. в [2].

ПРЕДЛОЖЕНИЕ 5.22. Пустъ $F$ - равномерно локально постоянный $\mathscr{J}(\mathbb{Z})$-модуль. Имеет место изоморфизм

$$
\widetilde{H}^{*}(\mathscr{J}(\mathbb{Z}) ; F)=\widehat{H}^{*}\left(K_{\infty} ; F\right) .
$$

Если $F$ - поле и тривиальный $\mathscr{J}(\mathbb{Z})$-модуль, то указанный изоморфизм будет изоморфизмом колец когомологий.

Как мы уже отмечали, пространство $K_{\infty}$ имеет достаточно сложную локальную структуру и в этом смысле сильно отличается от $C W$-комплексов. Тем не менее оно очень похоже на нильмногообразие. Следующий результат [2] можно рассматривать как бесконечномерный аналог известной теоремы Номидзу [85], связывающей вещественные когомологии некоторого нильмногообразия с когомологиями соответствующей нильпотентной алгебры Ли. "Нильмногообразию" $K_{\infty}=\mathscr{J}(\mathbb{R}) / \mathscr{J}(\mathbb{Z})$ естественно соответствует бесконечномерная топологическая алгебра Ли $W_{1}(1)$. Под когомологиями бесконечномерной алгебры Ли, как обычно, понимаются непрерывные когомологии (см. [49]).

ТЕорема 5.23. Имеет место изоморфизм колеи, вещественных когомологий

$$
\widehat{H}^{*}\left(K_{\infty} ; \mathbb{R}\right)=H^{*}\left(W_{1}(1) ; \mathbb{R}\right)
$$

Из двух последних утверждений непосредственно вытекает следующий результат.

СлеДСтвиЕ 5.24. Имеет место изоморфизм

$$
\widetilde{H}^{*}(\mathscr{J}(\mathbb{Z}) ; \mathbb{R}) \simeq H^{*}\left(W_{1}(1) ; \mathbb{R}\right) .
$$

Скажем еще несколько слов о вещественных когомологиях $\widetilde{H}^{*}(\mathscr{J}(\mathbb{Z}) ; \mathbb{R})$, которые, в силу сформулированных выше утверждений, удобно ассоциировать с когомологиями алгебры Ли $H^{*}\left(W_{1}(1) ; \mathbb{R}\right)$. 
Как было отмечено, например, в [50], [49], умножение в кольце $H^{*}\left(W_{1}(1) ; \mathbb{R}\right)$, а следовательно, и в кольце $\widetilde{H}^{*}(\mathscr{J}(\mathbb{Z}) ; \mathbb{R})$, тождественно нулевое. Это проявление градуировки самой алгебры Ли $W_{1}(1)$, что влечет биградуированность когомологий $H^{*}\left(W_{1}(1) ; \mathbb{R}\right)$. Вычисление второй градуировки групп когомологий (см. [50], [49]) показывает, что умножение тривиально по размерностным соображениям. В этой связи среди специалистов получила известность так называемая гипотеза Бухштабера, утверждающая, что все элементы $H^{k}\left(W_{1}(1) ; \mathbb{R}\right)$, $k>1$, разлагаются в линейные комбинации (нетривиальных, т. е. не содержащих нуля) произведений Масси, итерационно выраженных через одномерные базисные классы $\left[\omega_{1}\right],\left[\omega_{2}\right] \in H^{1}\left(W_{1}(1) ; \mathbb{R}\right)$. Здесь $\omega_{1}, \omega_{2} \in W_{1}(1)^{*}$ - два первых элемента базиса, двойственного к базису (31).

ПримеР 5.25. а) Трехместное произведение Масси $\left\langle\left[\omega_{1}\right],\left[\omega_{2}\right],\left[\omega_{2}\right]\right\rangle$ задает элемент в $H^{2}\left(W_{1}(1) ; \mathbb{R}\right)$ (это произведение автоматически будет нетривиальным).

b) Можно показать, что пятиместное произведение Масси $\left\langle\left[\omega_{1}\right],\left[\omega_{2}\right],\left[\omega_{1}\right]\right.$, $\left.\left[\omega_{1}\right],\left[\omega_{2}\right]\right\rangle$ также нетривиально и содержит элемент, дополняющий тройное произведение из а) до базиса в $H^{2}\left(W_{1}(1) ; \mathbb{R}\right)$.

Частичное решение гипотезы Бухштабера было получено в [45], где были явно построены семейства произведений Масси для базисных элементов в когомологиях. K сожалению, многие из этих произведений оказываются тривиальными. Еще одна попытка получить решение этой проблемы была предпринята в [1]. Однако полного базиса когомологий в этой работе получено не было, кроме того, в ней отсутствуют доказательства.

Наконец, эта гипотеза была недавно доказана в [79], где был указан рекуррентный (по размерности) процесс построения невырожденных произведений Масси, дающих базис в $H^{k}\left(W_{1}(1) ; \mathbb{R}\right)$. Исходные элементы этого процесса, порождающие $H^{2}\left(W_{1}(1) ; \mathbb{R}\right)$, указаны в примере 5.25. Заметим, что вес произведений Масси, получаемых в результате этого процесса, существенно выше размерности соответствующих когомологий.

5.9. Несколько замечаний о когомологиях группы $\mathscr{J}_{n}(\mathbb{Z})$. Вещественные когомологии $H^{*}\left(\mathscr{J}_{n}(\mathbb{Z}) ; \mathbb{R}\right)$ представляют независимый интерес и во многом более сложны, чем когомологии обратного предела $\widetilde{H}^{*}(\mathscr{J}(\mathbb{Z}) ; \mathbb{R})$. Поскольку $\mathscr{J}_{n}(\mathbb{Z})=\pi_{1}\left(K_{n}\right)$, то $H^{*}\left(\mathscr{J}_{n}(\mathbb{Z}) ; \mathbb{R}\right) \simeq H^{*}\left(K_{n} ; \mathbb{R}\right)$, где слева используются обычные когомологии дискретной группы, а справа - сингулярные когомологии пространства или когомологии де Рама конечномерного многообразия. Согласно теореме Номидзу [85], отсюда заключаем, что $H^{*}\left(\mathscr{J}_{n}(\mathbb{Z}) ; \mathbb{R}\right) \simeq$ $H^{*}\left(L_{n} ; \mathbb{R}\right)$, где $L_{n}$ - алгебра Ли, описанная в $(39)$. В отличие от $H^{*}\left(W_{1}(1) ; \mathbb{R}\right)$, когомологии $H^{*}\left(L_{n} ; \mathbb{R}\right)$ хоть и конечномерны, но имеют достаточно сложное умножение, более того, они являются алгеброй с двойственностью Пуанкаре. Естественное отображение

$$
H^{*}\left(L_{n} ; \mathbb{R}\right) \rightarrow H^{*}\left(W_{1}(1) ; \mathbb{R}\right),
$$

индуцированное проекцией $W_{1}(1) \rightarrow L_{n}$, имеет большое ядро. В настоящее время ни структура когомологий $H^{*}\left(L_{n} ; \mathbb{R}\right)$, ни ядро отображения $(41)$ не изучены, исключение составляют лишь размерности $\leqslant 3$. Результаты вычисления 
в размерностях 2 и 3 см. в [80]. Заключим этот пункт следующей элегантной гипотезой, принадлежащей Д. В. Миллионщикову.

ГиПотезА 5.26. При $k<n / 3$ для чисел Бетти алгебры Ли $L_{n}$ имеет место равенство

$$
b_{k}=\Phi_{k+2},
$$

где $\Phi_{k+2}-(k+2)$-е число Фибоначчи.

Можно показать непосредственным вычислением, что $H^{2}\left(L_{n}, \mathbb{R}\right)$ при $n>5$ порождено двумя стабильными классами когомологий, указанными в примеpe 5.25 , и нестабильным симплектическим классом $\left[\Omega_{n}\right]$. Вычисления размерности пространства $H^{k}\left(L_{n}, \mathbb{R}\right)(k \ll n)$ сильно усложняются с ростом $k$ и быстро становятся труднообозримыми.

\section{6. Подгруппы ростков голоморфных диффеоморфизмов в группах $\mathscr{J}(\mathbb{C})$ и $\mathscr{A}(\mathbb{C})$}

6.1. Однопараметрические подгруппы, классы сопряженных в $\mathscr{A}(\mathbb{C})$ и проблема Зигеля. Элементы вида

$$
f(x)=\alpha x+x \sum_{k=1}^{\infty} \alpha_{k} x^{k} \in \mathscr{A}(\mathbb{C}), \quad \alpha \in \mathbb{C}^{*},
$$

представимые сходящимся в некоторой окрестности нуля рядом, образуют подгруппу в $\mathscr{A}(\mathbb{C})$, которую мы обозначим $\mathscr{A}_{\mathrm{hol}}(\mathbb{C})$. Сходящиеся ряды $(42)$, для которых $\alpha=1$, образуют соответственно подгруппу $\mathscr{J}_{\text {hol }}(\mathbb{C})<\mathscr{J}(\mathbb{C})$. Эти подгруппы плотны относительно естественной топологии в $\mathscr{A}(\mathbb{C})$ и $\mathscr{J}(\mathbb{C})$ соответственно, инвариантны относительно гомотетий и согласованы со структурой полупрямого произведения $\mathscr{A}_{\mathrm{hol}}(\mathbb{C}) \simeq \mathbb{C}^{*} \ltimes \mathscr{J}_{\mathrm{hol}}(\mathbb{C})$.

Одна из основных проблем, стоявших в аналитической категории, состоит в изучении последовательности итераций $\left\{f^{n}(x)\right\}_{n=-\infty}^{\infty}$ некоторого элемента вида $(42)$ группы $\mathscr{A}_{\text {hol }}(\mathbb{C})$. На языке теории групп проблема заключается в изучении подгруппы, порожденной некоторым элементом $f(x) \in \mathscr{A}_{\mathrm{hol}}(\mathbb{C})$. Естественным расширением этой проблемы будет отыскание, если это возможно, однопараметрической подгруппы (потока)

$$
\left\{f_{\lambda}\right\}_{\lambda \in \mathbb{C}} \subset \mathscr{A}_{\mathrm{hol}}(\mathbb{C}), \quad \text { где } f_{\lambda} \circ f_{\mu}=f_{\lambda+\mu} \quad \text { и } \quad f_{\lambda}=f^{\lambda}, \quad \text { если } \quad \lambda \in \mathbb{Z} .
$$

Эта задача глубоко нетривиальна, сильно зависит от свойств мультипликатора $\alpha$ в (42) и показывает существенное различие аналитической и формальной категорий. Сюда же следует отнести и проблему описания классов сопряженных в группах $\mathscr{A}(\mathbb{C}), \mathscr{A}_{\text {hol }}(\mathbb{C})$ и $\mathscr{J}(\mathbb{C}), \mathscr{J}_{\text {hol }}(\mathbb{C})$ соответственно.

Естественным подходом к решению сформулированной выше задачи является отыскание соответствующей линеаризации (униформизации). Будем говорить, что формальный ряд $h(x) \in \mathscr{A}(\mathbb{C})$ линеаризует элемент $f(x)$, представленный рядом (42), если выполнено функциональное уравнение

$$
h(f(x))=\alpha h(x),
$$


часто называемое уравнением Шрёдера [95]. Структура полупрямого произведения на $\mathscr{A}(\mathbb{C})$ показывает, что если решение уравнения (44) существует, то его можно выбрать принадлежащим группе $\mathscr{J}(\mathbb{C})$. Поэтому линеаризующую функцию (решение уравнения Шрёдера) естественно искать среди функций, удовлетворяющих условию $h(x) \in \mathscr{J}(\mathbb{C})$. С помощью линеаризации удается также эффективно описать классы сопряженных элементов группы $\mathscr{A}(\mathbb{C})$ с нерезонансным мультипликатором.

В формальной категории и нерезонансной ситуации проблема линеаризации решается достаточно просто. Несложно показать, что если $\alpha$ не является корнем из единицы, то уравнение (44) имеет и притом единственное решение $h(x) \in \mathscr{J}(\mathbb{C})$. Более того, можно показать, что если $\alpha$ не является корнем из единицы, то для коэффициентов ряда $h(x)=x\left(1+\sum_{n=1}^{\infty} h_{n} x^{n}\right)$, представляющего линеаризацию, имеет место формула

$$
h_{n-1}=\frac{1}{\alpha^{n}-\alpha}\left(\alpha_{n-1}+\sum_{q=2}^{n-1} \alpha_{q-1} \sum_{\substack{j_{1}+\cdots+j_{q}=n-q \\ j_{i} \geqslant 0}} h_{j_{1}} \cdots h_{j_{q}}\right),
$$

где $h_{0}=1$.

Заметим, что проблема формальной линеаризации формального отображения $\mathbb{R}^{n} \rightarrow \mathbb{R}^{n}$ с неподвижным началом координат хорошо известна и восходит, видимо, к Пуанкаре. В нерезонансном случае проблема имеет положительное решение. Эта тематика не имеет прямого отношения к теме настоящей работы, и мы ее здесь не обсуждаем, отсылая интересующегося читателя к [15], где собрана обширная библиография в данном направлении. См. также [102], где решается задача гладкой линеаризации гладкого нерезонансного отображения.

Легко видеть, что если (формальная) линеаризация существует, то однопараметрическая подгруппа (43) задается простой формулой

$$
f_{\lambda}(x)=h^{-1}(x) \circ\left(\alpha^{\lambda} x\right) \circ h(x) .
$$

В нерезонансной ситуации линеаризация позволяет ответить и на следующий вопрос: Какие элементы группы $\mathscr{A}(\mathbb{C})$ коммутируют с данным элементом вида (42)? Действительно, несложно показать, что если $\alpha$ не является корнем из единицы, то любой элемент группы $\mathscr{A}(\mathbb{C})$, коммутирующий с $f(x)$, принадлежит формальному непрерывному семейству (46).

Заметим, что однопараметрическая подгруппа (46), рассматриваемая как отображение $\mathbb{C}$ в $\mathscr{A}(\mathbb{C})$, соответствующее параметру $\lambda$, всегда имеет нетривиальное ядро и, в этом смысле, отличается от однопараметрических подгрупп в $\mathscr{J}(\mathbf{k})$, рассматривавшихся выше.

Вопрос об аналитичности линеаризации $h(x)$ и, следовательно, семейства $f_{\lambda}(x)$ существенно более деликатен, чем вопрос о ее формальном существовании. Один из первых результатов, описывающий гиперболический случай, был получен еще в XIX веке Кёнигсом [68].

ТЕОРема 6.1. Если мулътипликатор элемента $f(x)$, заданного сходящимся в нуле рядом (42), удовлетворяет условию $|\alpha| \neq 1$, то линеаризация $h(x) \in$ $\mathscr{J}(\mathbb{C})$ однозначно определена и аналитична в некоторой окрестности 0. 
Если $\alpha=\mathbf{e}^{2 \pi i t}$, где фаза $t \in \mathbb{R}$ иррациональна, то, как мы видели выше, линеаризация $h(x)$ однозначно определена как формальный ряд, коэффициенты которого вычисляются по формуле (45). В этом случае, как было показано Кремером и Зигелем в первой половине прошлого века, сходимость ряда $h(x)$ зависит от арифметических свойств параметра $t$.

Обозначим $\Lambda=\left\{\alpha=\mathbf{e}^{2 \pi i t} \mid t \in \mathbb{R} \backslash \mathbb{Q}\right\}$ множество нерезонансных мультипликаторов, лежащих на единичной окружности. Первый результат о существовании расходящейся линеаризации у сходящегося ряда (42) с мультипликатором $\alpha \in \Lambda$ был получен почти сто лет назад Пфайфером [91]. Используя соображения типа общего положения, Пфайфер доказал, что существует “достаточно много" мультипликаторов $\alpha \in \Lambda$ и таких сходящихся рядов (42), отвечающих этим мультипликаторам, что соответствующая линеаризация (44) задается расходящимся рядом. В [91] Пфайфер высказал (ошибочное) предположение, что "кажется правдоподобным", что для всех мультипликаторов из $\Lambda$ существуют примеры аналитических $f(x)$, имеющие расходящуюся линеаризацию. Тем не менее мультипликаторы, возникавшие в его конструкции, аномально быстро приближались корнями из единицы и, следовательно, их было достаточно мало в смысле меры. Заметим еще, что результат Пфайфера являлся чистой теоремой существования, ни одного конкретного примера предъявлено не было.

Позже Кремер [28] доказал, что для всех мультипликаторов $\alpha \in \Lambda$, удовлетворяющих условию

$$
\liminf _{n \rightarrow \infty}\left|\alpha^{n}-1\right|^{1 / n}=0
$$

существуют аналитические в окрестности 0 отображения (42) с расходящейся линеаризацией. Он также сделал важное наблюдение о том, что расходимость линеаризации имеет место уже для полиномиальных отображений.

Мультипликаторы, удовлетворяющие условию (47), хорошо аппроксимируются корнями из единицы, и их мера на единичной окружности равна нулю. Здесь следует отметить, что еще раньше Жюлиа [61] пытался доказать, что линеаризация всегда расходится, если $f(x)-\alpha x-$ ненулевая рациональная функция, а фаза $t$ мультипликатора $\alpha$ иррациональна. Однако эта гипотеза оказалась неверна, что вытекает из следующего общего результата Зигеля [99].

Tеорема 6.2. Пусть $\ln \left|\alpha^{n}-1\right|=\mathscr{O}(\ln n), n \rightarrow \infty$. Тогда для любого сходящегося ряда (42) с данным мультипликатором а решение уравнения Шрёдера сходится.

В терминах фазы условие теоремы эквивалентно неравенству

$$
\left|t-\frac{m}{n}\right|>r n^{-s}
$$

для любых целых $n, m$, где $r$ и $s$ - положительные числа, зависящие только от $t$. На единичной окружности такие мультипликаторы образуют множество полной меры.

Таким образом, множество всех нерезонансных мультипликаторов делится на два непустых непересекающихся подмножества:

$$
\Lambda=C \cup S, \quad C \cap S=\varnothing,
$$


характеризующихся следующим образом. Если $\alpha \in C$, то существует сходящийся ряд (42) с мультипликатором $\alpha$, для которого решение уравнения Шрёдера расходится. Если же $\alpha \in S$, то любой сходящийся в некоторой окрестности 0 ряд (42) с данным мультипликаторм $\alpha$ имеет сходящуюся линеаризацию. Теорема Зигеля описывает в $S$ некоторое подмножество, имеющее полную меру на всей окружности. Проблема полного описания множеств $C$ и $S$ получила название проблемы Зигеля, иногда ее также называют проблемой аналитичности решения уравнения Шрёдера.

Оказалось, что наиболее удобная форма арифметических условий Кремера (47) и Зигеля (48) может быть дана в терминах цепной дроби, представляющей фазу $t$ мультипликатора $\alpha$. Уточним обозначения. Для числа $t \in \mathbb{R} \backslash \mathbb{Q}$ обозначим $t_{0}=[t] \in \mathbb{Z}$ и $\tau_{0}=\{t\} \in(0,1)$ соответственно целую и дробную части этого числа. Определим индуктивно $t_{n}=\left[\tau_{n-1}^{-1}\right]$ и $\tau_{n}=\left\{\tau_{n-1}^{-1}\right\}, n=1,2, \ldots$. Далее, как обычно, положим $q_{-2}=p_{-1}=1, q_{-1}=p_{-2}=0$ и индуктивно определим последовательность натуральных чисел

$$
q_{n}=t_{n} q_{n-1}+q_{n-2}, \quad p_{n}=t_{n} p_{n-1}+p_{n-2}, \quad n=1,2, \ldots
$$

Будем говорить, что последовательность рациональных чисел $\left\{p_{n} / q_{n}\right\}_{n=0}^{\infty} a c$ соииирована с мультипликатором $\alpha=\mathbf{e}^{2 \pi i t} \in \Lambda$. Эта последовательность сходится к фазе $t$ и состоит из $n$-членных редукций цепной дроби, в которую разлагается $t$. При этом четная подпоследовательность $\left\{p_{2 k} / q_{2 k}\right\}_{k=0}^{\infty}$ монотонно возрастает, а нечетная $\left\{p_{2 k+1} / q_{2 k+1}\right\}_{k=0}^{\infty}$ монотонно убывает. Доказательство следующего предложения можно найти, например, в [113].

ПрЕДЛОжЕНИЕ 6.3. Пусть $\left\{q_{n}\right\}_{n=0}^{\infty}-$ знаменатели ассоииированной с мультипликатором $\alpha \in \Lambda$ последовательности. Тогда условие Кремера (47) эквивалентно условию

$$
\sup _{n} \frac{\ln q_{n+1}}{q_{n}}=+\infty,
$$

а условие Зигеля (48) эквивалентно условию

$$
\sup _{n} \frac{\ln q_{n+1}}{\ln q_{n}}<+\infty .
$$

Следующий результат дает решение проблемы Зигеля.

Теорема 6.4. Для того чтобы мультипликатор $\alpha \in \Lambda$ принадлежал множеству $S$, необходимо и достаточно, чтобы знаменатели $\left\{q_{n}\right\}_{n=0}^{\infty}$ ассоциированной с ним последовательности удовлетворяли условию

$$
\sum_{n=0}^{\infty} \frac{\ln q_{n+1}}{q_{n}}<+\infty .
$$

Условие (50) было сформулировано Брюно [17], [18], и им же была доказана его достаточность. Необходимость этого условия была установлена Йокозом [114], [113]. Развивая идею Кремера о нелинеаризуемости полиномиальных отображений, в этих же работах Йокоз доказал, что если $\alpha \in C$, то в качестве нелинеаризуемого отображения (42) с данным мультипликатором достаточно 
взять квадратичный многочлен. Точнее, им была доказана следующая теорема.

Теорема 6.5. Если $\alpha \in \Lambda$ и квадратичный полином $\alpha z(1-z)$ имеет аналитическую линеаризачию, то любая аналитическая функиия вида $\alpha z+\mathscr{O}\left(z^{2}\right)$ имеет аналитическую линеаризацию.

Доказательства теорем Йокоза связаны с интересными проблемами комплексной динамики, которые лежат уже достаточно далеко от темы настоящей статьи, и мы их не касаемся. Обширную библиографию в этом направлении можно найти, например, в [90] и [15].

Очевидно, что мультипликатор элемента вида (42) является инвариантом класса сопряженных этого элемента, как в группе $\mathscr{A}(\mathbb{C})$, так и в $\mathscr{A}_{\text {hol }}(\mathbb{C})$. Из представленных выше результатов вытекает, что если $\alpha \in \mathbb{C}^{*} \backslash\left(C \cup\left\{z^{n}=1\right\}_{n=1}^{\infty}\right)$, то это единственный инвариант класса сопряженных в обеих группах. Иначе говоря, любой элемент вида $\alpha z+\mathscr{O}\left(z^{2}\right)$, как в группе $\mathscr{A}(\mathbb{C})$, так и в $\mathscr{A}_{\mathrm{hol}}(\mathbb{C})$, сопряжен своей линейной части $\alpha z$.

Если $\alpha \in C$, то в $\mathscr{A}(\mathbb{C})$ все элементы вида $\alpha z+\mathscr{O}\left(z^{2}\right)$ все еще лежат в одном классе, определяемом $\alpha z$, а в $\mathscr{A}_{\mathrm{hol}}(\mathbb{C})$ это уже не так и точное описание классов сопряженных остается неясным. Сложность строения классов сопряженных в группе $\mathscr{A}_{\mathrm{hol}}(\mathbb{C})$ для отображений с мультипликаторами из множества Кремера $C$ объясняется сложным поведением периодических орбит таких отображений в окрестности нуля. Изучая строение периодических орбит и скорость их приближения к нулю, Йокоз [113], в частности, получил следующий результат. Пусть $H_{\alpha} \subset \mathscr{A}_{\mathrm{hol}}(\mathbb{C})$ - множество сходящихся в некоторой окрестности нуля рядов (42), имеющих фиксированный мультипликатор $\alpha$. Очевидно, что $H_{\alpha}$ либо представляет один класс сопряженных в $\mathscr{A}_{\mathrm{hol}}(\mathbb{C})$, либо распадается на классы сопряженных, целиком лежащие в $H_{\alpha}$.

Теорема 6.6. Если $\alpha \in C$, то в $H_{\alpha}$ имеется несчетное подмножество классов сопряженных, причем ни один из этих классов не содержит ни одной иелой функиии.

Наконец, кратко рассмотрим случай, когда мультипликатор $\alpha$ является корнем из единицы. Если бы линеаризация (44) была возможна, то элемент $f(x)$ был бы элементом конечного порядка, равного степени $\alpha$ как примитивного корня из единицы. Оказывается, что это условие также достаточно для наличия формальной и аналитической линеаризации в рассматриваемом случае. Действительно, если $f^{m}(x)=x$ в $\mathscr{A}(\mathbb{C})$, где $m$ - наименьшее натуральное число с этим свойством, то $\alpha$ - примитивный корень из единицы степени $m$ и по крайней мере одно решение $h(x) \in \mathscr{J}(\mathbb{C})$ уравнения Шрёдера $(44)$ всегда существует:

$$
h(x)=\frac{1}{m} \sum_{k=0}^{m-1} \alpha^{-k} f^{k}(x) .
$$

Формула (51) показывает, что если в сделанных предположениях $f(x) \in \mathscr{A}_{\text {hol }}(\mathbb{C})$, то $h(x)$ также может быть выбрана аналитичной в нуле. Наконец, заметим, что в рассматриваемом случае решений уравнения Шрёдера бесконечно много, а формула (51) дает, в каком-то смысле, каноническое решение. Легко 
проверить, что если $\alpha$ - примитивный корень из единицы степени $m$ и $h(x)-$ некоторая линеаризация $f(x)$, то любой элемент $g(x) \circ h(x)$, где $g(x) \in \mathscr{J}_{(m)}(\mathbb{C})$ (эта подгруппа была определена в (17)), также будет линеаризовать $f(x)$.

Рассмотренное выше необходимое и достаточное условие линеаризации показывает, что "почти все" элементы вида (42), где $\alpha$ - корень из единицы, не допускают линеаризации. Несмотря на отсутствие линеаризации элементов (42), мультипликатор которых - корень из единицы, классы сопряженных в группе $\mathscr{A}(\mathbb{C})$ устроены все еще сравнительно просто. Как доказано в [83], справедливо следующее предложение.

ПРЕДЛОЖЕниЕ 6.7. Если $\alpha$ - первообразный коренъ из единищъ степени $m$ u $f^{m}(x) \neq x$, mо $f(x)$ сопряжен в $\mathscr{A}(\mathbb{C})$ элементу вида

$$
f_{n, a}(x)=\alpha x\left(1+x^{n m}+a x^{2 n m}\right)
$$

при подходящих $n \in \mathbb{N} u a \in \mathbb{C}$. Элементь $f_{n, a}(x)$ u $f_{n^{\prime}, a^{\prime}}(x)$ сопряжень в $\mathscr{A}(\mathbb{C})$ тогда и только тогда, когда $n=n^{\prime} u$ u $a=a^{\prime}$.

Иначе говоря, отличные от $\{\alpha x\}$ классы сопряженных занумерованы двумя параметрами $n \in \mathbb{N}$ и $a \in \mathbb{C}$.

6.2. Однопараметрические подгруппы и классы сопряженных в группах $\mathscr{J}(\mathbb{C})$ и $\mathscr{J}_{\text {hol }}(\mathbb{C})$. Теперь рассмотрим случай $\alpha=1$, т.е. $f(x) \in$ $\mathscr{J}(\mathbb{C})$. С одной стороны, в этой ситуации линеаризация уже невозможна. С другой стороны, сформулированная в начале раздела проблема о непрерывном семействе $f_{\lambda}$, содержащем $f(x)$, просто решается в формальной категории с помощью экспоненциального отображения. Действительно, как мы видели в п. 4.4, существует единственный формальный ряд $v(x)=\sum_{k=1}^{\infty} \alpha_{k}^{\prime} x^{k+1}$ такой, что $\exp \left(v(x) \frac{d}{d x}\right)=f(x)$. Искомое семейство является однопараметрической подгруппой, проходящей через элемент $f(x)$, и задается формулой

$$
f_{\lambda}(x)=\exp \left(\lambda v(x) \frac{d}{d x}\right)(x) .
$$

Как следует из предложения 2.15 , все элементы $\mathscr{J}(\mathbb{C})$, коммутирующие с $f(x)$, также принадлежат семейству (52). Какие элементы большей группы $\mathscr{A}(\mathbb{C})$ могут коммутировать с данным $f(x) \in \mathscr{J}(\mathbb{C})$, проанализировано в [10], мы этот вопрос не обсуждаем.

Все вышесказанное приводит нас к следующей интересной и глубоко нетривиальной проблеме. Рассмотрим некоторый элемент $f(x) \in \mathscr{J}(\mathbb{C})$ и соответствующую ему однопараметрическую подгруппу $f_{\lambda}(x)$. Пусть $K\left(f_{\lambda}\right) \subseteq \mathbb{C}-$ подмножество точек комплексной $\lambda$-плоскости, для которых ряд $f_{\lambda}(x)$ имеет положительный радиус сходимости. Естественный вопрос - какова структура множества $K\left(f_{\lambda}\right)$ ?

Этот вопрос был детально изучен Бейкером [10] в 60-х годах прошлого века, им, в частности, были получены следующие неожиданные результаты. 
ТЕОрема 6.8. Для любого элемента $f(x) \in \mathscr{J}(\mathbb{C})$ соответствующее множество $K=K\left(f_{\lambda}\right)$ может быть лишь одного из следующих четырех видов:

a) $K=\{0\}$;

b) $K=\left\{n \lambda_{0}\right\}$, где $n \in \mathbb{Z} u \lambda_{0} \neq 0$ (комплексная решетка ранга 1 );

c) $K=\left\{n \lambda_{0}+m \lambda_{1}\right\}$, где $n, m \in \mathbb{Z} u \lambda_{0}, \lambda_{1}$ - вещественно несоизмеримые комплексные числа (комплексная решетка ранга 2 );

d) $K=\mathbb{C}$.

Теорема 6.9. Случаи a), b) и d) предыдущей теоремы реализуются.

Реализация возможностей а) и d) несложна и может быть рассмотрена читателем как соответствующее упражнение. Реализация возможности b) - гораздо более тонкий вопрос. Первым таким примером был найденный Бейкером [9] ряд $f(x)=\mathbf{e}^{x}-1$, представляющий целую функцию. Им было показано, что в семействе $f_{\lambda}(x)=x+\lambda x^{2} / 2+\cdots$ лишь целые итерации $f^{n}(x)$ соответствуют сходящимся рядам (в данном случае это целые функции). Таким образом, в рассматриваемом примере $K\left(f_{\lambda}\right)=\mathbb{Z} \subset \mathbb{C}$. Позже рядом авторов были найдены многочисленные другие функции $f(x)$, реализующие возможность $\mathrm{b})$. В частности, как было установлено в [105], сюда относятся все целые функции вида $f(x)=x+\mathscr{O}\left(x^{2}\right)$, а также рациональные функции такого же вида, исключая, естественно, $x /(1-x)$.

Реализуемость формальной возможности с) долгое время оставалась неясной. Наконец, Экалем [36] было показано, что множества $K\left(f_{\lambda}\right)$ типа с) не peaлизуются. Этот результат был позже независимо передоказан Ворониным [108].

Теорема 6.8 имеет следующую геометрическую интерпретацию. Если $V(x)=$ $v(x) d / d x$ - некоторый касательный вектор в единице группы $\mathscr{J}(\mathbb{C})$, то однопараметрическая подгруппа в данном направлении либо пересекает подгруппу $\mathscr{J}_{\text {hol }}(\mathbb{C})$ всего в одной точке $x$ или по некоторой (бесконечной циклической) подгруппе, либо полностью содержится в $\mathscr{J}_{\text {hol }}(\mathbb{C})$. Этот последний случай был изучен Эрдёшем и Жаботинским [40].

ТЕОРема 6.10. Однопараметрическая подгруппа, заданная формулой (52), целиком лежит в группе $\mathscr{J}_{\mathrm{hol}}(\mathbb{C})$ тогда и только тогда, когда соответствующий ей касательный вектор задается сходящимся в некоторой окрестности нуля рядом $v(x)$.

Теоремы 6.8 и 6.10 показывают, что вложение $\mathscr{J}_{\text {hol }}(\mathbb{C}) \subset \mathscr{J}(\mathbb{C})$ достаточно сложно. Экспоненциальное отображение переводит подалгебру локально голоморфных векторных полей $W_{1}^{\text {hol }}(1) \subset W_{1}(1)$ в группу $\mathscr{J}_{\text {hol }}(\mathbb{C})$, но при этом оно не является сюръективным. Обозначим

$$
\mathscr{J}_{\text {hol }}^{\mathrm{reg}}(\mathbb{C})=\exp \left(W_{1}^{\mathrm{hol}}(1)\right) \quad \text { и } \quad \mathscr{J}_{\text {hol }}^{\mathrm{res}}(\mathbb{C})=\mathscr{J}_{\text {hol }}(\mathbb{C}) \backslash \mathscr{J}_{\text {hol }}^{\mathrm{reg}}(\mathbb{C}) .
$$

Таким образом, в группе $\mathscr{J}_{\text {hol }}(\mathbb{C})$ имеется большое исключительное подмножество элементов $\mathscr{J}_{\text {hol }}^{\text {res }}(\mathbb{C})$, через которые не проходят однопараметрические подгруппы этой группы. Отметим, что при этом группа $\mathscr{J}_{\text {hol }}(\mathbb{C})$ стягивается по себе в точку, такое стягивание можно осуществить, например, с помощью гомотетий. 
Теорема 6.10 дает необходимые и достаточные условия на касательный вектор $V(x)=v(x) d / d x$, при которых элемент $f(x)=\exp (V(x))$ принадлежит подмножеству $\mathscr{J}_{\mathrm{hol}}^{\mathrm{reg}}(\mathbb{C})$. Необходимые условия на $V(x)$, при которых $f(x)=$ $\exp (V(x))$ принадлежит $\mathscr{J}_{\mathrm{hol}}^{\text {res }}(\mathbb{C})$, были найдены Жаботинским [58].

Теорема 6.11. Пусть $f(x)=\exp \left(\left(\sum_{k=1}^{\infty} \alpha_{k}^{\prime} x^{k+1}\right) \frac{d}{d x}\right)$ принадлежст $\mathscr{J}_{\text {hol }}^{\mathrm{res}}(\mathbb{C})$. Тогда

$$
\limsup _{k \rightarrow \infty}\left|\alpha_{k}^{\prime}\right|^{1 / k}=\infty \quad u \quad \limsup _{k \rightarrow \infty} \frac{1}{k}\left|\alpha_{k}^{\prime}\right|^{1 / k}<\infty .
$$

Будут ли эти условия достаточными, иначе говоря, будет ли при выполнении этих условий однопараметрическая подгруппа $f_{\lambda}(x)=\exp (\lambda V(x))$ пересекать $\mathscr{J}_{\text {hol }}(\mathbb{C})$ в неединичных элементах - неизвестно.

Как подгруппа $\mathscr{J}(\mathbb{C})$, группа $\mathscr{J}_{\text {hol }}(\mathbb{C})$, очевидно, обладает ОИК-свойством, однако само наличие корня из некоторого элемента уже не всегда имеет место в $\mathscr{J}_{\text {hol }}(\mathbb{C})$. Более точно, для любого элемента подмножества $\mathscr{J}_{\mathrm{hol}}^{\mathrm{reg}}(\mathbb{C})$ извлечение корня любой степени всегда возможно. Для элементов, содержащихся в $\mathscr{J}_{\text {hol }}^{\text {res }}(\mathbb{C})$, извлечение корней отсутствует за исключением, возможно, конечного числа показателей. В [37] Экалем был построен функциональный инвариант, позволяющий для данного $f(x) \in \mathscr{J}_{\text {hol }}(\mathbb{C})$ и натурального $n$ ответить на вопрос, существует ли (итерационный) корень степени $n$ из $f(x)$, обсуждение этого инварианта см. ниже. Аналогичный инвариант был позже независимо определен в [108]. Заметим, что практическое применение этого инварианта к конкретной аналитической в окрестности 0 функции вида $f(x)=x+\mathscr{O}\left(x^{2}\right)$ весьма затруднительно.

Как показывает следующий результат Экаля [35], подмножество $\mathscr{J}_{\mathrm{hol}}^{\mathrm{reg}}(\mathbb{C}) \subset$ $\mathscr{J}_{\text {hol }}(\mathbb{C})$ достаточно "тощее" и совершенно незамкнуто относительно групповой структуры в $\mathscr{J}_{\text {hol }}(\mathbb{C})$.

Teорема 6.12. Пусть $g(x) \in \mathscr{J}_{\mathrm{hol}}(\mathbb{C}) u f(x) \in \mathscr{J}_{\mathrm{hol}}^{\mathrm{reg}}(\mathbb{C})-$ не коммутирующий с ним элемент, причем $f_{\lambda}(x)$ - отвечающая этому элементу однопараметрическая подгруппа (52). Тогда лебегова мера тех $\lambda \in \mathbb{C}$, для которых $g \circ f_{\lambda}\left(f_{\lambda} \circ g\right)$ лежит в $\mathscr{J}_{\mathrm{hol}}^{\mathrm{reg}}(\mathbb{C})$, равна нулю.

Интересно отметить, что хотя в $\mathscr{J}_{\text {hol }}^{\text {reg }}(\mathbb{C})$ лежат многие алгебраические функции, например, $x\left(1-x^{n}\right)^{-1 / n}, n=1,2, \ldots$, функции вида "алгебраическая плюс мероморфная" уже попадают в $\mathscr{J}_{\text {hol }}^{\text {res }}(\mathbb{C})($ см. [35]).

Классы сопряженных в группе $\mathscr{J}(\mathbb{C})$ устроены достаточно просто и их описание не составляет большого труда. Следующий результат аналогичен предложению 6.7 и также доказан в [83].

ПрЕДЛОЖЕНИЕ 6.13. Каждый элемент группы $\mathscr{J}(\mathbb{C})$ сопряжен в ней элементу вида

$$
x\left(1+b x^{m}+a x^{2 m}\right),
$$

где а и $b$ - комплексные числа. Параметры $m \in \mathbb{N} u a, b \in \mathbb{C}$ однозначно определяют класс сопряженных элементов.

Формальная нормальная форма (53) устанавливалась очень многими авторами, и, возможно, работа [83] не была самой ранней. Заметим, что наличие 
здесь параметра $b$, по сравнению с нормальной формой предложения 6.7 , объясняется тем, что сопряжение берется только элементами из $\mathscr{J}(\mathbb{C})$, т. е. гомотетии исключены.

Несложно видеть, что множество $\mathscr{J}_{\mathrm{hol}}^{\mathrm{reg}}(\mathbb{C})$ состоит из полных классов сопряженных группы $\mathscr{J}_{\text {hol }}(\mathbb{C})$. Как было установлено в [83], голоморфная и формальная сопряженность элементов из $\mathscr{J}_{\mathrm{hol}}^{\mathrm{reg}}(\mathbb{C})$ совпадают.

ПреДЛОЖЕНИЕ 6.14. Для любого элемента $f \in \mathscr{J}_{\mathrm{hol}}^{\mathrm{reg}}(\mathbb{C})$ имеет место равенство

$$
f^{\mathscr{J}_{\text {hol }}(\mathbb{C})}=f^{\mathscr{J}(\mathbb{C})} \cap \mathscr{J}_{\text {hol }}(\mathbb{C}) .
$$

ЗАмЕчАНИЕ 6.15. Здесь мы сталкиваемся со следующим парадоксальным явлением: два элемента $f(x)$ и $g(x)$ из множества $\mathscr{J}_{\text {hol }}^{\text {reg }}(\mathbb{C})$ сопряжены в группе $\mathscr{J}_{\text {hol }}(\mathbb{C})$ тогда и только тогда, когда они имеют одну и ту же формальную нормальную форму (53). Вместе с тем сам элемент $(53)$ им в $\mathscr{J}_{\text {hol }}(\mathbb{C})$ не сопряжен, поскольку согласно [105] лежит в $\mathscr{J}_{\text {hol }}^{\text {res }}(\mathbb{C})$. Наконец, отметим, что каждый класс сопряженных в группе $\mathscr{J}(\mathbb{C})$ содержит элемент из множества $\mathscr{J}_{\mathrm{hol}}^{\mathrm{reg}}(\mathbb{C})$. Имеет место равенство

$$
\left(\mathscr{J}_{\text {hol }}^{\text {reg }}(\mathbb{C})\right)^{\mathscr{J}(\mathbb{C})}=\left(\mathscr{J}_{\text {hol }}^{\text {res }}(\mathbb{C})\right)^{\mathscr{J}(\mathbb{C})}=\mathscr{J}(\mathbb{C}) .
$$

Наконец, кратко обсудим функциональный инвариант классов сопряженных группы $\mathscr{J}_{\text {hol }}(\mathbb{C})$, первоначально введенный и изученный Экалем в [36] и [37]. В самом общем случае, когда $f(x)=x\left(1+c x^{n}+\mathscr{O}\left(x^{n+1}\right)\right)$, этот инвариант состоит из $n$ пар функций, аналитичных в полуплоскостях $\operatorname{Im} z>K(f)$ и $\operatorname{Im} z<-K(f)$ для некоторой подходящей положительной константы $K(f)$, периодичных с периодом 1 и определяемых некоторым специальным образом по $f(x)$. В целом эта конструкция слишком громоздка для изложения в обзорной статье. Ситуация разумно упрощается в случае общего положения, т. е. $n=1$, который был позже независимо изучен Ворониным [108].

Пусть $\varepsilon= \pm 1$, рассмотрим пару рядов

$$
\Phi_{\varepsilon}(\xi)=\xi+\sum_{k=0}^{\infty} c_{k}^{\varepsilon} \mathbf{e}^{\varepsilon 2 \pi i k \xi},
$$

сходящихся соответственно в полуплоскостях $\varepsilon \operatorname{Im} \xi>N$ для некоторого $N>0$. На множестве таких пар введем отношение эквивалентности. Будем говорить, что пара $\Phi_{\varepsilon}(\xi)$ эквивалентна паре $\widetilde{\Phi}_{\varepsilon}(\xi)$, если найдутся комплексные $c_{1}$ и $c_{2}$ такие, что $\Phi_{\varepsilon}\left(\xi+c_{1}\right)=\widetilde{\Phi}_{\varepsilon}\left(\xi+c_{2}\right)$ для всех $\xi$ таких, что $\varepsilon \operatorname{Im} \xi>N_{1}$, где $N_{1}>N$. Множество классов эквивалентности $\mathscr{M}_{\text {gen }}$ пар рядов $(54)$ и составляет пространство модулей аналитической сопряженности подмножества $\mathscr{J}_{\mathrm{hol}}(\mathbb{C})$, состоящего из рядов общего положения (имеющих вид $\left.x\left(1+\alpha_{1} x+\mathscr{O}\left(x^{2}\right)\right), \alpha_{1} \neq 0\right)$. Строго говоря, как Экалем, так и Ворониным изучалось сопряжение элементов группы $\mathscr{J}_{\text {hol }}(\mathbb{C})$ элементами из объемлющей группы $\mathscr{A}_{\mathrm{hol}}(\mathbb{C})$. Это совершенно логично с точки зрения анализа (общие замены переменных), но неестественно с точки зрения теории групп. Поэтому сформулированная далее теорема содержит дополнительное условие, исключающее гомотетии. Нижеследующий результат является адаптацией результатов [36], [37] к подмножеству $\mathscr{J}_{\text {hol }}(\mathbb{C})$ $\bmod \mathscr{J}^{2}(\mathbb{C})$ и соответствует центральному результату работы [108]. 
Tеорема 6.16. Существует сюргективное отображение $f(x) \mapsto m_{f}$ подмножества элементов $f \in \mathscr{J}_{\mathrm{hol}}(\mathbb{C})$, нетривиальных $\bmod \mathscr{J}^{2}(\mathbb{C})$, на множество $\mathscr{M}_{\mathrm{gen}}$, постоянное на классах сопряженных. Более того,

$$
f(x)^{\mathscr{J}_{\text {hol }}(\mathbb{C})}=g(x)^{\mathscr{J}_{\text {hol }}(\mathbb{C})}
$$

тогда и толъко тогда, когда $m_{f}=m_{g} u f \equiv g \bmod \mathscr{J}^{2}(\mathbb{C})$.

В заключение отметим, что набор обсуждаемых функциональных инвариантов позволяет также ответить на вопрос, возможно ли в группе $\mathscr{J}_{\text {hol }}(\mathbb{C})$ из данного элемента $f$ извлечь корень данной степени $n$ (детали см. в [36], [37] и [108]).

\section{7. Группа $\mathscr{J}(\mathbf{k})$ и когомологические операции}

7.1. Инвариантные дифференциальные операторы на группе $\mathscr{J}(\mathbf{k})$. Глобальные канонические координаты $(2)$ на группе $\mathscr{J}(\mathbf{k})$ удобны для изучения пространств непрерывных функций на $\mathscr{J}(\mathbf{k})$ со значениями в основном кольце $\mathbf{k}$, а также их алгебраических аналогов. В этом разделе в качестве естественного пространства таких функций рассматривается кольцо многочленов от канонических координат $\mathbf{k}[\boldsymbol{\alpha}]=\mathbf{k}\left[\alpha_{1}, \alpha_{2}, \ldots\right]$ с коэффициентами в $\mathbf{k}$. Заметим, что в случае поля $\mathbf{k}$ характеристики нуль полиномы могут быть отождествлены с соответствующими функциями и $\mathbf{k}[\boldsymbol{\alpha}]$ можно рассматривать как подпространство в $C^{\infty}(\mathscr{J}(\mathbf{k}))$. По-видимому, впервые важность кольца $\mathbf{k}[\boldsymbol{\alpha}]$ полиномов на группе $\mathscr{J}(\mathbf{k})$ была установлена Бухштабером и Шокуровым [22].

В кольце $\mathbf{k}[\boldsymbol{\alpha}]$ удобно ввести градуировку, считая, что образующая $\alpha_{k}$ имеет степень $2 k$. Эта градуировка естественна и согласуется с однородностью закона умножения (4) в $\mathscr{J}(\mathbf{k})$. Кольцо $\mathbf{k}[\boldsymbol{\alpha}]$ становится градуированной $\mathbf{k}$-алгеброй. При любой заданной топологии основного кольца $\mathbf{k}$ топология на $\mathbf{k}[\boldsymbol{\alpha}]$ вводится как топология прямого предела

$$
\mathbf{k}[\boldsymbol{\alpha}]=\underset{n}{\lim } \mathbf{k}\left[\alpha_{1}, \alpha_{2}, \ldots, \alpha_{n}\right] .
$$

Все рассматриваемые далее отображения всегда будут непрерывны, и мы не будем это специально подчеркивать. Алгебраическая природа объектов в этом пункте более важна, чем топология.

Алгебра $\mathbf{k}[\boldsymbol{\alpha}]$ является (топологической) алгеброй Хопфа, где коумножение $\Delta$ на образующих определяется формулами (4), двойственными к умножению на группе, а для коединицы, естественно, имеем $\varepsilon\left(\alpha_{n}\right)=0$.

Следуя [22], рассмотрим общую алгебраическую конструкцию, связанную с некоторой структурой алгебры Хопфа $(\mathbf{k}[\boldsymbol{\alpha}], \Delta)$, заданной на алгебре полиномов. Используя изоморфизм $\mathbf{k}[\boldsymbol{\alpha}] \otimes \mathbf{k}[\boldsymbol{\alpha}] \simeq \mathbf{k}[\boldsymbol{\alpha}, \boldsymbol{\beta}]=\mathbf{k}\left[\alpha_{1}, \beta_{1}, \alpha_{2}, \beta_{2}, \ldots\right]$, удобно рассматривать коумножение как отображение алгебр

$$
\Delta_{\alpha}^{\beta}: \mathbf{k}[\boldsymbol{\alpha}] \rightarrow \mathbf{k}[\boldsymbol{\alpha}, \boldsymbol{\beta}]
$$

Сохраняя терминологию [22], отображение (55) будем называть сдвигом. 
ПримеР 7.1. Положим на образующих $\Delta_{\boldsymbol{\alpha}}^{\boldsymbol{\beta}}\left(\alpha_{k}\right)=\alpha_{k}+\beta_{k}, k=1,2, \ldots$. Полученная так алгебра Хопфа будет соответствовать алгебре полиномов на абелевой группе $\mathbf{k}^{\mathbb{N}}$ с операцией покоординатного сложения.

Далее будет удобно использовать следующие обозначения. Пусть $\mathbb{Z}_{+}$обозначает множество неотрицательных целых чисел, положим $\mathbb{Z}_{+}^{\infty}=\lim _{n} \mathbb{Z}_{+}^{n}$. Таким образом, элементами полугруппы $\mathbb{Z}_{+}^{\infty}$ являются бесконечные последовательности $\mathbf{n}=\left(n_{1}, n_{2}, \ldots\right)$ неотрицательных целых чисел, равных 0 начиная с некоторого места; положим $\mathbf{n} !=n_{1} ! n_{2} ! \cdots$ и $|\mathbf{n}|=\sum_{i=1}^{\infty}\left|n_{i}\right|$. Обозначим $\boldsymbol{\alpha}^{\mathbf{n}}=\alpha_{1}^{n_{1}} \alpha_{2}^{n_{2}} \ldots$ соответствующий полином алгебры $\mathbf{k}[\boldsymbol{\alpha}]$.

Рассмотрим символы $\left\{\partial_{1}, \partial_{2}, \ldots\right\}$, и пусть

$$
D=\mathbf{k}[\boldsymbol{\alpha}]\left[\left[\partial_{1}, \partial_{2}, \ldots\right]\right]=\mathbf{k}[\boldsymbol{\alpha}] \otimes_{\mathbf{k}} \mathbf{k}\left[\left[\partial_{1}, \partial_{2}, \ldots\right]\right]
$$

- кольцо формальных степенных рядов с коэффициентами в алгебре полиномов $\mathbf{k}[\boldsymbol{\alpha}]$. Это кольцо биградуировано естественным образом, образующие $\alpha_{n}$ имеют биградуировку $\operatorname{deg}_{1}=(2 n, 0)$, а $\partial_{n}-$ биградуировку $\operatorname{deg}_{2}=(0,-2 n)$. Для изучения кольца $D$ полезны общая степень $\operatorname{deg}=\operatorname{deg}_{1}+\operatorname{deg}_{2}$ и убывающая фильтрация $\mathscr{F}_{2 n}(D)=\left\{d \in D \mid \operatorname{deg}_{2}(d) \leqslant-2 n\right\}$.

Кольцо $D$ естественно рассматривать как кольцо дифференциальных операторов на алгебре $\mathbf{k}[\boldsymbol{\alpha}]$. Любой элемент $d \in D$ запишется в виде $d=\sum_{\mathbf{n} \in \mathbb{Z}_{+}^{\infty}} q_{\mathbf{n}}(\boldsymbol{\alpha}) \partial^{\mathbf{n}}$, где $q_{\mathbf{n}}(\boldsymbol{\alpha}) \in \mathbf{k}[\boldsymbol{\alpha}]$. Действие $d$ на алгебре функций $\mathbf{k}[\boldsymbol{\alpha}]$ задается очевидной формулой

$$
d(p(\boldsymbol{\alpha}))=\sum_{\mathbf{n} \in \mathbb{Z}_{+}^{\infty}} q_{\mathbf{n}}(\boldsymbol{\alpha}) \partial^{\mathbf{n}}(p(\boldsymbol{\alpha}))=\sum_{\mathbf{n} \in \mathbb{Z}_{+}^{\infty}} q_{\mathbf{n}}(\boldsymbol{\alpha}) \frac{\partial^{n_{1}}}{\partial \alpha_{1}^{n_{1}}} \frac{\partial^{n_{2}}}{\partial \alpha_{2}^{n_{2}}} \cdots p(\boldsymbol{\alpha}) .
$$

Заметим, что для любого $p(\boldsymbol{\alpha}) \in \mathbf{k}[\boldsymbol{\alpha}]$ сумма в (56) уже конечна. Таким образом, каждый элемент $d \in D$ задает эндоморфизм $\mathbf{k}[\boldsymbol{\alpha}]$ как $\mathbf{k}$-модуля. Естественным умножением в $D$ является операторное умножение, превращающее $D$ в ассоциативную (но не коммутативную) k-алгебру.

C помощью дифференциальных операторов алгебры $D$ удобно выражать другие, более сложные операторы.

ПримеР 7.2. Несложно проверить, что для оператора сдвига примера 7.1 имеет место формула

$$
\Delta_{\boldsymbol{\alpha}}^{\boldsymbol{\beta}} p(\boldsymbol{\alpha})=\sum_{\mathbf{n}} \frac{1}{\mathbf{n} !} \partial^{\mathbf{n}}(p(\boldsymbol{\alpha})) \boldsymbol{\beta}^{\mathbf{n}}
$$

где $p(\boldsymbol{\alpha}) \in \mathbf{k}[\boldsymbol{\alpha}]$.

В случае произвольного кольца $\mathbf{k}$ оператор (57) следует понимать как единое целое. Если $\mathbf{k}$ содержит поле рациональных чисел, то (57) можно рассматривать как произведение операторов первого порядка в алгебре $D$.

Пусть теперь $\boldsymbol{\Delta}_{\boldsymbol{\alpha}}^{\boldsymbol{\beta}}$ - произвольный сдвиг (55) на алгебре полиномов. Будем говорить, что оператор $d \in D$ инвариантен относительно сдвига $\boldsymbol{\Delta}_{\boldsymbol{\alpha}}^{\boldsymbol{\beta}}$, если 
$\Delta_{\boldsymbol{\alpha}}^{\boldsymbol{\beta}}(d(p))=d_{\boldsymbol{\beta}}\left(\Delta_{\boldsymbol{\alpha}}^{\boldsymbol{\beta}}(p)\right)$ для любого полинома $p \in \mathbf{k}[\boldsymbol{\alpha}]$. Здесь $d_{\boldsymbol{\beta}}$ обозначает такой $\mathbf{k}[\boldsymbol{\alpha}]$-гомоморфизм кольца $\mathbf{k}[\boldsymbol{\alpha}, \boldsymbol{\beta}]$ в себя, что $\left.d_{\boldsymbol{\beta}}(p(\boldsymbol{\beta}))\right|_{\boldsymbol{\beta}=\boldsymbol{\alpha}}=d(p(\boldsymbol{\alpha}))$. Обозначим $\mathscr{D} \subset D$ подкольцо операторов, инвариантных относительно сдвига $\boldsymbol{\Delta}_{\boldsymbol{\alpha}}^{\boldsymbol{\beta}}$. Обобщая формулу (57), сам сдвиг $\Delta_{\boldsymbol{\alpha}}^{\boldsymbol{\beta}}$ можно представить в виде

$$
\Delta_{\boldsymbol{\alpha}}^{\boldsymbol{\beta}}(p(\boldsymbol{\alpha}))=\sum_{\mathbf{n}} \mathscr{D}_{\mathbf{n}}(p(\boldsymbol{\alpha})) \boldsymbol{\beta}^{\mathbf{n}}
$$

где $p(\boldsymbol{\alpha}) \in \mathbf{k}[\boldsymbol{\alpha}]$ - произвольный полином, а $\mathscr{D}_{\mathbf{n}}-$ некоторые элементы кольца $D$. Следующее утверждение доказано в [22].

ПреДЛОЖениЕ 7.3. Пусть $\Delta_{\boldsymbol{\alpha}}^{\boldsymbol{\beta}}$ - оператор сдвига (55) на алгебре полиномов. Тогда все операторы $\mathscr{D}_{\mathbf{n}}, \mathbf{n} \in \mathbb{Z}_{+}^{\infty}$, его разложения (58) принадлежат подкольиу $\mathscr{D}$, причем $\mathscr{D}_{\mathbf{0}}=1$.

По существу, инвариантность этих операторов выражает свойство ассоциативности коумножения в соответствующей алгебре Хопфа $(\mathbf{k}[\boldsymbol{\alpha}], \Delta)$. Операторы $\mathscr{D}_{\mathbf{n}}, \mathbf{n} \in \mathbb{Z}_{+}^{\infty}$, называются каноническими инвариантными операторами на коалгебре $(\mathbf{k}[\boldsymbol{\alpha}], \Delta)$.

Рассмотрим двойственную алгебру Хопфа $\mathbf{k}[\boldsymbol{\alpha}]^{*}=\operatorname{Hom}_{\mathbf{k}}(\mathbf{k}[\boldsymbol{\alpha}], \mathbf{k})$, умножение в ней двойственно коумножению в $(\mathbf{k}[\boldsymbol{\alpha}], \Delta)$. Аугментация (коединица) $\varepsilon$ определяет гомоморфизм k-модулей

$$
\varepsilon^{*}: D \rightarrow \mathbf{k}[\boldsymbol{\alpha}]^{*}
$$

по формуле $\varepsilon^{*}(d)=\varepsilon \circ d, d \in D$. Доказательство следующего утверждения можно найти в [19].

ПреДЛОЖенИЕ 7.4. а) Для любого $d \in \mathscr{D}$ справедливо представление

$$
d=\sum_{\mathbf{n}} \varepsilon\left(d\left(\boldsymbol{\alpha}^{\mathbf{n}}\right)\right) \mathscr{D}_{\mathbf{n}}
$$

b) Ограничение $\varepsilon^{*}$ на подкольцо $\mathscr{D} \subset D$ индущирует кольщевой изоморфизм

$$
\varepsilon^{*}: \Delta \rightarrow \mathbf{k}[\boldsymbol{\alpha}]^{*}
$$

Оказывается, что если сдвиг $\Delta_{\boldsymbol{\alpha}}^{\boldsymbol{\beta}}$ соответствует умножению в группе $\mathscr{J}(\mathbf{k})$, то в кольце $D$ операторы $\mathscr{D}_{\mathbf{n}}$ однородны относительно общей степени, причем $\operatorname{deg}\left(\mathscr{D}_{\mathbf{n}}\right)=-2|\mathbf{n}|$. Кроме того, имеет место равенство

$$
\mathscr{D}_{\mathbf{n}}=\frac{1}{\mathbf{n} !} \partial^{\mathbf{n}}+\mathscr{D}_{\mathbf{n}}^{\prime}
$$

где $\mathscr{F}\left(\mathscr{D}_{\mathbf{n}}^{\prime}\right)<-2|\mathbf{n}|$.

Вернемся к случаю $\mathbf{k}=\mathbb{R}$; несложно проверить, что операторы $\mathscr{D}_{\mathbf{n}}, \mathbf{n} \in \mathbb{Z}_{+}^{\infty}$, оставляют подкольцо целочисленных полиномов $\mathbb{Z}[\boldsymbol{\alpha}] \subset \mathbb{R}[\boldsymbol{\alpha}]$ инвариантным. Кроме того, рассматриваемые как операторы на группе Ли $\mathscr{J}(\mathbb{R})$, они оказываются левоинвариантными дифференциальными операторами. Обозначим $\mathscr{D}_{\mathbb{Z}} \subset \mathscr{D}$ подмножество целочисленных операторов из $\mathscr{D}$, т. е. оставляющих кольцо $\mathbb{Z}[\boldsymbol{\alpha}]$ инвариантным. Из предложения 7.4 следует, что операторы $\left\{\mathscr{D}_{\mathbf{n}}\right\}_{\mathbf{n} \in \mathbb{Z}_{+}^{\infty}}$ образуют аддитивный базис $\mathbb{Z}$-алгебры $\mathscr{D}_{\mathbb{Z}}$. 
Положим $\mathbf{1}(k)=(0, \ldots, 0,1,0, \ldots) \in \mathbb{Z}_{+}^{\infty}$, где единица стоит на $k$-м месте. Операторы $\mathscr{D}_{\mathbf{1}(k)}, k=1,2, \ldots$, представляют особый интерес. Пусть $e_{k}=x^{k+1} \frac{d}{d x}$ - элемент канонического базиса алгебры Ли $L$ (см. (31)). Тогда соответствующее ему левоинвариантное векторное поле на $\mathscr{J}(\mathbb{R})$ задает целочисленный дифференциальный оператор первого порядка, совпадающий c $\mathscr{D}_{1(k)}$ :

$$
\mathscr{D}_{\mathbf{1}(k)}=\partial_{k}+\sum_{s>k}(s-k+1) \alpha_{s-k} \partial_{s}
$$

Для алгебры инвариантных дифференциальных операторов $\mathscr{D}$ справедлив вариант теоремы Л. Шварца (см. [63]), устанавливающей изоморфизм алгебр (Хопфа) $\mathscr{D} \simeq U(L)$, где $U(L)$ - универсальная обертывающая алгебра алгебры Ли $L$ группы $\mathscr{J}(\mathbb{R})$. В рассматриваемом случае изоморфизм будет иметь место над любым полем $\mathbf{k}$ характеристики 0. Иначе говоря, справедлив изоморфизм алгебр Хопфа

$$
\mathbf{k} \otimes_{\mathbb{Z}} \mathscr{D}_{\mathbb{Z}} \simeq U_{\mathbf{k}}(L),
$$

где $L$ следует рассматривать как $\mathbf{k}$-алгебру Ли с тем же базисом (31).

Наконец, определим еще одно подкольцо кольца $D$ дифференциальных операторов на группе $\mathscr{J}(\mathbb{R})$. Рассмотрим элемент $\phi(x)=1-\mathbf{e}^{-x} \in \mathscr{J}(\mathbb{R})$, и пусть $\mathscr{U} \subset \mathbb{Z}[\boldsymbol{\alpha}]$ - подкольцо целочисленных полиномов на группе $\mathscr{J}(\mathbb{R})$, которые правоинвариантны относительно $\phi(x)$. Имеется естественный мономорфизм

$$
\mathscr{U} \otimes_{\mathbb{Z}} \mathscr{D}_{\mathbb{Z}} \rightarrow D
$$

образ которого является левым $\mathscr{U}$-модулем. Обозначим $\mathscr{D} \mathscr{U}$ подкольцо $D$, порожденное этим $\mathscr{U}$-модулем.

7.2. Когомологические операции. Рассмотрим некоторую теорию когомологий $\mathscr{H}^{*}$ на категогии конечных клеточных комплексов. В качестве основных примеров мы имеем в виду теорию обычных (сингулярных) когомологий $H^{*}\left(, \mathbb{Z}_{p}\right)$ с коэффициентами в $\mathbb{Z}_{p}$ или теорию комплексных кобордизмов $\mathrm{U}^{*}($ ) (см., например, [103]). Напомним, что когомологической операцией степени $s$ называется набор отображений

$$
\alpha_{i}^{X}: \mathscr{H}^{i}(X) \rightarrow \mathscr{H}^{i+s}(X)
$$

заданный для каждого конечного клеточного комплекса $X$ и всех $i \in \mathbb{Z}$. Причем эти отображения естественны, т. е. для любого непрерывного отображения $f: X \rightarrow Y$ двух комплексов диаграмма

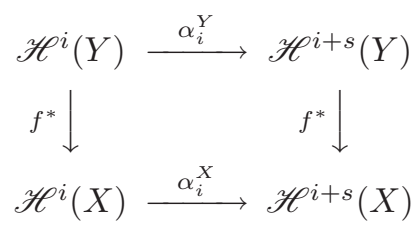


коммутативна. Когомологическая операция называется стабилъной, если она коммутирует с оператором надстройки: для любого комплекса $X$ коммутативна диаграмма

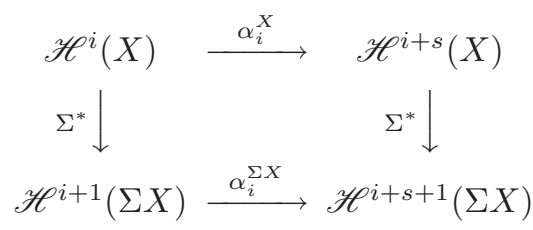

Иначе говоря, стабильные когомологические операции это операции, перестановочные с точной последовательностью пары $(X, Y)$ комплексов. Здесь, как обычно, считается, что $\mathscr{H}^{*}(X, Y) \simeq \widetilde{\mathscr{H}^{*}}(X / Y)$.

Далее мы рассматриваем лишь стабильные операции. Заметим, что хотя сами отображения $\alpha_{i}^{X}$ не обязаны быть гомоморфизмами, их можно складывать между собой, поскольку они принимают значения в абелевой группе. Это превращает множество операций данной степени $s$ в абелеву группу, а множество $\mathscr{A}_{\mathscr{H}}$ всех стабильных операций теории $\mathscr{H}^{*}$ в градуированную абелеву группу. Умножение в $\mathscr{A}_{\mathscr{H}}$ задается композицией операций $(\alpha \beta)^{X}=\alpha^{X} \circ \beta^{X}$, что превращает $\mathscr{A}_{\mathscr{H}}$ в ассоциативное (но не коммутативное) градуированное кольцо. Кольцо $\mathscr{A} \mathscr{H}$ считается уже пополненным относительно своей естественной градуировки. Кроме того, из определения немедленно следует, что для каждого комплекса $X$ его когомологии $\mathscr{H}^{*}(X)$ становятся модулем над алгеброй когомологических операций $\mathscr{A}_{\mathscr{H}}$.

Алгебры операций, возникающие для двух отмеченных выше теорий когомогий, хорошо известны. Это алгебра Стинрода $\mathscr{A}_{p}$ в случае когомологического функтора $H^{*}\left(, \mathbb{Z}_{p}\right)$ (см. [101]) и кольцо операций $\mathscr{A}^{U}$ в унитарных кобордизмах $\mathrm{U}^{*}()$. Это кольцо непосредственно связано с группой $\mathscr{J}(\mathbb{R})$.

Кольцо $\mathscr{A}^{U}$ может быть описано в виде свободного левого модуля над кольцом кобордизмов комплексных многообразий $\Omega_{U}^{*}$ с базисом из некоторой алгебры Хопфа $S$. Для любого комплекса $X$ элементы $\sigma \in \Omega_{U}^{*}$ действуют в $\mathrm{U}^{*}(X)$ как умножение на соответствующий элемент подкольца точки $\mathrm{U}^{*}(\mathrm{pt}) \subset \mathrm{U}^{*}(X)$, выбранный с помощью естественного изоморфизма $\mathrm{U}^{*}(\mathrm{pt}) \simeq \Omega_{U}^{*}$. Каждый элемент $\sigma \in \Omega_{U}^{r}$ задает операцию степени $-r$. Напомним, что, согласно теореме Милнора-Новикова, кольцо $\Omega_{U}^{*}$ изоморфно кольцу полиномов $\mathbb{Z}\left[\alpha_{1}, \alpha_{2}, \ldots\right]$, где образующая $\alpha_{k}$ рассматривается как элемент степени $2 k$.

Строение подкольца $S \subset \mathscr{A}^{U}$ играет существенную роль в изучении строения всего кольца операций $\mathscr{A}^{U}$. Его структура впервые изучалась в работах Ландвебера [70] и Новикова [86], теперь это кольцо называется алгеброй Ландвебера-Новикова. Структура кольца $S$ достаточно сложная. Как было показано в работах [70] и [86], в $S$ можно выбрать специальный базис $s_{\mathbf{n}}, \mathbf{n} \in \mathbb{Z}_{+}^{\infty}$, где $\mathbb{Z}_{+}^{\infty}$ было введено в предыдущем пункте после примера 7.1. Элементы $s_{\mathbf{n}}$ строятся при помощи классов Чженя в комплексных кобордизмах (на их конструкции мы не останавливаемся) и задают когомологические операции степени $2|\mathbf{n}|$. В [70] и [86] была доказана следующая важная теорема.

Теорема 7.5. Подгруппа $S \subset \mathscr{A}^{U}$, порожденная операциями $s_{\mathbf{n}}, \mathbf{n} \in \mathbb{Z}_{+}^{\infty}$, замкнута относительно композиции. Отображение $\Omega_{U}^{*} \otimes S \rightarrow \mathscr{A}^{U}$, заданное формулой $\sigma \otimes s \mapsto \sigma \circ s$, мономорфно и его образ всюду плотен в $\mathscr{A}^{U}$. 
Правила коммутирования $\sigma \circ s=s \circ \sigma^{\prime}, s \in S, \sigma, \sigma^{\prime} \in \Omega_{U}^{*}$, были впервые найдены Новиковым [86] и важны при вычислениях, например, гомотопических групп.

Связь алгебры Ландвебера-Новикова $S$ с группой $\mathscr{J}(\mathbb{R})$ была установлена Бухштабером и Шокуровым [22], в частности, ими была доказана следующая теорема.

Теорема 7.6. Алгебра Ландвебера-Новикова $S$ изоморфна кольцу $\mathscr{D}_{\mathbb{Z}}$ иелочисленных левоинвариантных дифференциальных операторов на группе $\mathscr{J}(\mathbb{R})$. Этот изоморфизм задается соответствием $s_{\mathbf{n}} \mapsto \mathscr{D}_{\mathbf{n}}, \mathbf{n} \in \mathbb{Z}_{+}^{\infty}$.

Следующие два утверждения также получены в [22]. Первое вытекает непосредственно из теоремы 7.6 и (59).

СлЕДСТВИЕ 7.7. Имеет место изоморфизм алгебр

$$
S \otimes \mathbf{k} \simeq U_{\mathbf{k}}(L)
$$

где $\mathbf{k}$ - поле характеристики 0.

С использованием проведенных в [50] вычислений когомологий алгебры $L=$ $W_{1}(1)$ в [22] было получено следующее утверждение.

СлеДСТвиЕ 7.8. Справедливо равенство

$$
\operatorname{Ext}_{S}^{n, k}(\mathbb{Q}, \mathbb{Q})= \begin{cases}\mathbb{Q}, & \text { если } k=3 n^{2} \pm n \\ 0, & \text { если } k \neq 3 n^{2} \pm n\end{cases}
$$

В работе [22] также была дана интерпретация алгебры всех когомологических операций $\mathscr{A}^{U}$ как подкольца в кольце дифференциальных операторов на группе $\mathscr{J}(\mathbb{R})$.

Теорема 7.9. Существует изоморфизм колеи, $\mathscr{A}^{U} \rightarrow \mathscr{D}_{\mathscr{U}}$, продолюсающий изоморфизм теоремы 7.6 подкольиа $S$ на $\mathscr{D}_{\mathbb{Z}}$.

Интерпретация алгебры Стинрода $\mathscr{A}_{p}$ в терминах групп диффеоморфизмов также существует и тоже была найдена Бухштабером [20]. Это, однако, приводит к необходимости рассмотрения так называемых "суперрасширений" групп $\mathscr{J}\left(\mathbb{Z}_{p}\right)$ и выходит за рамки настоящей статьи, детали можно найти в работе [20]. Появление нечетных суперобразующих связано с тем, что $H^{*}\left(X, \mathbb{Z}_{p}\right)$ $(p>2)$ кроме четномерной уже имеет и нетривиальную нечетномерную часть. Таким образом, действие $\mathscr{A}_{p}$ в $H^{*}\left(X, \mathbb{Z}_{p}\right)$ уже следует рассматривать как представление в $\mathbb{Z}_{p}$-векторном суперпространстве.

Тем не менее один из полученных в [20] результатов имеет непосредственное выражение в терминах групп $\mathscr{J}(\mathbf{k})$, им мы и завершим настоящий раздел. Когомологическая операция называется мультипликативной, если она действует на кольцах когомологий пространства как кольцевой гомоморфизм. Алгебра $\mathscr{A}_{p}$ содержит единицу, и это позволяет говорить о группе обратимых элементов этой алгебры. Мультипликативные операции из этой группы образуют так называемую подгруппу всех мультипликативных операций. 
Теорема 7.10. Индексная подгруппа $\mathscr{D}<\mathscr{J}\left(\mathbb{Z}_{p}\right)$ примера 4.24 изоморфна подгруппе всех мультипликативных операций обычной теории когомологий $H^{*}\left(, \mathbb{Z}_{p}\right)$.

\section{8. Аменабельность}

\section{1. Несколько замечаний об аменабельности не локально ком-} пактных групп. Пусть $G$ - группа, которую мы всегда рассматриваем как топологическую группу; если изначально топология не уточнена, то считаем ее дискретной. Пространство всех непрерывных ограниченных функций на $G$ обозначим $C^{\star}(G)$, соответственно через $C_{\mathrm{ru}}^{\star}(G)$ обозначим подпространство всех ограниченных равномерно непрерывных справа функций на $G$. Оба пространства наделяются нормой $L_{\infty}$ и становятся банаховыми пространствами.

Левоинвариантным средним (ЛИС) на пространстве $C^{\star}(G)$ (или $\left.C_{\mathrm{ru}}^{\star}(G)\right)$ называется непрерывный линейный функционал $F$, определенный на этом пространстве и обладающий следующими свойствами:

$$
\begin{cases}F(f) \geqslant 0, \text { если } f \geqslant 0 & \text { (положительность); } \\ F(1)=1 & \text { (нормировка); } \\ F\left({ }_{h} f\right)=F(f), h \in G & \text { (инвариантность), }\end{cases}
$$

где ${ }_{h} f(g)=f(h g)$. Под аменабельностью группы $G$ понимается существование ЛИС на соответствующем пространстве $C^{\star}(G)$ (или $C_{\mathrm{ru}}^{\star}(G)$ ). Эквивалентные определения, а также детальное введение в предмет можно найти в [53] или [89]. Мы лишь отметим, что свойство аменабельности устойчиво относительно взятия подгруппы и сюръективного образа. Оно также сохраняется при переходе от некоторой аменабельной группы к ее расширению с помощью другой аменабельной группы. К основным примерам аменабельных групп относятся абелевы группы, что вместе со свойством устойчивости при расширениях приводит к аменабельности всех разрешимых групп. Основным примером неаменабельной группы является свободная группа ранга 2 с дискретной топологией. Сохранение аменабельности при переходе к подгруппам влечет за собой неаменабельность любой группы, содержащей дискретную свободную некоммутативную подгруппу.

Для дискретных групп пространства $C^{\star}(G)$ и $C_{\mathrm{ru}}^{\star}(G)$ совпадают с пространством $l_{\infty}(G)$ всех ограниченных на $G$ функций, и оба вида аменабельности, очевидным образом, не различаются. Хорошо известно [53; теорема 2.2.1], что для локально компактной группы $G$ существование ЛИС на пространстве $C_{\mathrm{ru}}^{\star}(G)$ влечет существование среднего на пространстве $C^{\star}(G)$ (обратное очевидно для любой группы $G$ ). Таким образом, для локально компактных групп оба типа аменабельности также совпадают. Для не локально компактных групп это, вообще говоря, неверно (см. [31]) и выбор подходящего пространства функций становится достаточно деликатной проблемой.

Для коммутативного кольца с единицей $\mathrm{k}$ имеется канонический гомоморфизм $i: \mathbb{Z} \rightarrow \mathbf{k}$, заданный равенством $i(n)=n \mathbf{1}$. Если $\operatorname{ker} i \neq 0$, то $\mathbf{k}$ содержит подкольцо, изоморфное $\mathbb{Z}_{p}$ для некоторого простого $p$, и, следовательно, группа $\mathscr{J}(\mathbf{k})$ содержит подгруппу, изоморфную $\mathscr{J}\left(\mathbb{Z}_{p}\right)$. В этом случае из 
теоремы 4.6 следует существование в $\mathscr{J}(\mathbf{k})$ свободной подгруппы индекса 2. В случае $\operatorname{ker} i=0$ группа $\mathscr{J}(\mathbf{k})$ содержит $\mathscr{J}(\mathbb{Z})$ как подгруппу и наличие в $\mathscr{J}(\mathbf{k})$ свободной подгруппы индекса 2 вытекает из следствия 5.10. Таким образом, на пространстве $l_{\infty}(\mathscr{J}(\mathbf{k}))$ всех ограниченных функций, определенных на группе $\mathscr{J}(\mathbf{k})$, левоинвариантное среднее отсутствует, и мы видим, что $\mathscr{J}(\mathbf{k})$, рассматриваемая как дискретная группа, не является аменабельной. Это показывает, что при изучении возможных инвариантных средних на группе $\mathscr{J}(\mathbf{k})$ важно учитывать ее естественную топологию и исходя из этого выбирать пространство ограниченных функций, на котором существовало бы такое инвариантное среднее.

В случае компактного (например, конечного) кольца $\mathbf{k}$ группа $\mathscr{J}(\mathbf{k})$ также компактна и вопрос об инвариантных средних на пространстве непрерывных функций положительно решается в рамках теории меры Хаара. В случае некомпактного (например, бесконечного дискретного) кольца $\mathbf{k}$, согласно теореме 2.19 , группа $\mathscr{J}(\mathbf{k})$ может не вкладываться ни в какую локально компактную группу. Для таких колец $\mathbf{k}$ наиболее естественно искать ЛИС на пространстве равномерно непрерывных функций на группе $\mathscr{J}(\mathbf{k})$.

Для общих топологических групп $G$, в отличие от локально компактного случая, существование ЛИС именно на пространстве $C_{\mathrm{ru}}^{\star}(G)$ приобретает первостепенную важность благодаря следующему общему результату (теорема 8.2 ниже), который следует считать классическим. Прежде чем его сформулировать, напомним несколько определений. Будем говорить, что топологическая группа $G$ обладает свойством неподвижной точки, если для любого выпуклого компакта $K$ в некотором локально выпуклом топологическом векторном пространстве $E$ любое непрерывное аффинное действие $G$ на $K$ обладает неподвижной точкой. Через $\gamma_{\mathrm{u}}(G)$ будем обозначать максимальное компактное эквивариантное расширение группы $G$. Пространство $\gamma_{\mathrm{u}}(G)$ называется также эквивариантной компактификацией группы $G$, это же пространство известно как компактификация Самюэля [94], отвечающая правой равномерности на группе $G$.

В качестве возможной конструкции этого расширения можно взять следующую (детали и соответствующие свойства этого расширения см. в [55], [77]). В пространстве $\left(C_{\mathrm{ru}}^{\star}(G)\right)^{*}$, двойственном к $C_{\mathrm{ru}}^{\star}(G)$, рассмотрим единичный шар $B$ в *-слабой топологии. Имеется естественное вложение $i: G \rightarrow B$, определяемое формулой $i(g)(f)=f(g), g \in G, f \in C_{\mathrm{ru}}^{\star}(G)$. Пусть $\gamma_{\mathrm{u}}(G)=\overline{i(G)}-$ замыкание образа этого вложения. Компактность пространства $\gamma_{\mathrm{u}}(G)$ вытекает из компактности $B$ в $*$-слабой топологии. Основные свойства компактификации $\gamma_{\mathrm{u}}(G)$ приведены в следующем предложении.

ПРЕДЛОЖЕНИЕ 8.1. а) Естественное вложение $G \subset \gamma_{\mathrm{u}}(G)$ индущирует изоморфизм пространств функиий $C_{\mathrm{ru}}^{\star}(G) \simeq C\left(\gamma_{\mathrm{u}}(G)\right)$.

b) Пусть $X \supset G$ - компакт, в котором $G$ всюду плотна, и левое действие $G$ на себе продолжается до непрерьвного действия $G$ на $X$. Тогда существует непрерывное эквивариантное отображсение $f: \gamma_{\mathrm{u}}(G) \rightarrow X$, тождественное на $G$ (свойство максимальности).

c) Если группа $G$ дискретна, то $\gamma_{\mathrm{u}}(G) \simeq \beta(G)$. 
Здесь $\beta(G)$ - стоун-чеховская компактификация топологического пространства $G$ (см., например, [27], [34]).

Теорема 8.2. Для топологической группы $G$ следующие условия эквивалентны:

а) на пространстве $C_{\mathrm{ru}}^{\star}(G)$ существует ЛИС;

b) группа $G$ обладает свойством неподвижной точки;

c) любое непрерьвное действие $G$ на любом компакте обладает инвариантной вероятностной мерой;

d) естественное действие группы $G$ на своем максимальном эквивариантном компактном расширении $\gamma_{\mathrm{u}}(G)$ обладает инвариантной вероятностной мерой.

Эквивалентность условий b) и с) теоремы 8.2 в основном доказана Деем [29], [30]. Формулировка для общих топологических групп и соответствующее доказательство, использующее идеи Дея, принадлежат Риккерту [93]. Классический результат Боголюбова [16] утверждает, что наличие ЛИС на пространстве $C^{\star}(G)$ всех непрерывных и ограниченных функций на группе $G$ влечет за собой наличие инвариантной меры для любого непрерывного действия $G$ на компакте. В действительности для существования инвариантных мер некоторого непрерывного действия $G$ на компакте $X$ достаточно потребовать существование ЛИС лишь на пространстве $C_{\text {ru }}^{\star}(G)$. Такие меры в точности являются неподвижными точками ассоциированного действия $G$ на компакте вероятностных мер на $X$, и их существование обеспечено результатом Дея-Риккерта. С другой стороны, существование инвариантной меры для любого непрерывного действия $G$ на компакте влечет, в частности, существование такой меры для естественного действия $G$ на своей равномерной компактификации $\gamma_{\mathrm{u}}(G)$. Поскольку пространства функций $C_{\mathrm{ru}}^{\star}(G)$ и $C\left(\gamma_{\mathrm{u}}(G)\right)$ естественно изоморфны, интеграл по любой инвариантной мере на $\gamma_{\mathrm{u}}(G)$ определяет инвариантное среднее на пространстве $C_{\mathrm{ru}}^{\star}(G)$. Укажем, что эквивалентность условий с) и d) теоремы 8.2 можно получить и непосредственно из свойства максимальности равномерной компактификации $\gamma_{\mathrm{u}}(G)$.

8.2. Аменабельность $\mathscr{J}(\mathbf{k})$. Несложно видеть, что в случае группы $G=$ $\mathscr{J}(\mathbf{k})$ левая и правая равномерности совпадают. Таким образом, мы можем рассматривать просто ограниченные равномерно непрерывные функции на группе $\mathscr{J}(\mathbf{k})$, пространство которых обозначим $C_{\mathrm{u}}^{\star}(\mathscr{J}(\mathbf{k}))$. Двусторонние инвариантные средние, в отличие от ЛИС, называем далее инвариантными средними. Следующий результат получен в [4].

ТеОрема 8.3. Пусть $\mathbf{k}$ - коммутативное топологическое кольцо с единицей. На пространстве $C_{\mathrm{u}}^{\star}(\mathscr{J}(\mathbf{k}))$ равномерно непрерывных ограниченных функиий на группе $\mathscr{J}(\mathbf{k})$ существует инвариантное среднее.

Отсюда и из теоремы 8.2 вытекает следующее утверждение.

СлеДСтвиЕ 8.4. Пусть $\mathbf{k}$ - коммутативное топологическое кольцо с единицей. Любое непрерывное действие группы $\mathscr{J}(\mathbf{k})$ на любом компакте обладает инвариантной вероятностной мерой. 
ПримеР 8.5. Компакт $K_{\infty}$ определен в (36) как пространство правых смежных классов. Таким образом, $\mathscr{J}(\mathbb{R})$ естественно действует на $K_{\infty}$ левыми сдвигами. Несложно показать, что это действие непрерывно и что вероятностная мера $\mathbf{m}$ в теореме 5.16 инвариантна относительно этого действия.

Рассмотрим базисное кольцо $\mathbf{k}$, снабженное некоторой топологией $\theta$, и пусть $\theta_{0}$ - дискретная топология на том же кольце. Топологии, возникающие на $\mathscr{J}(\mathbf{k})$, обозначим соответственно $\Theta$ и $\Theta_{0}$. Очевидное включение пространств функций

$$
C_{\mathrm{u}}^{\star}(\mathscr{J}(\mathbf{k}) ; \Theta) \subset C_{\mathrm{u}}^{\star}\left(\mathscr{J}(\mathbf{k}) ; \Theta_{0}\right)
$$

показывает, что из существования среднего на пространстве $C_{\mathrm{u}}^{\star}\left(\mathscr{J}(\mathbf{k}) ; \Theta_{0}\right)$ вытекает наличие среднего на пространстве ограниченных равномерно непрерывных функций, ассоциированных с любой другой топологией $\theta$, заданной на $\mathbf{k}$. Иначе говоря, для изучения аменабельности групп $\mathscr{J}(\mathbf{k})$ наибольший интерес представляют как раз дискретные кольца. Отметим, что существование ЛИС на пространстве всех непрерывных ограниченных функций $C^{\star}(\mathscr{J}(\mathbf{k}))$ остается неясным даже в случае $\mathbf{k}=\mathbb{Z}$.

Дискретные кольца $\mathbf{k}$ интересны и с точки зрения динамики, связанной с группой $\mathscr{J}(\mathbf{k})$. Рассмотрим действие $\mathscr{J}(\mathbf{k})$ на своей стоун-чеховской компактификации $\beta(\mathscr{J}(\mathbf{k}))$.

ЗАмЕчАниЕ 8.6. Если $G$ - топологическая группа, то ее действие на себе левыми сдвигами продолжается до действия гомеоморфизмами на ее стоун-чеховской компактификации $\beta(G)$. Вообще говоря, это действие может быть как разрывным, так и несвободным. В случае дискретной группы $G$ это действие является непрерывным. Известный результат Эллиса [38] утверждает, что для дискретной группы $G$ ее действие на $\beta(G)$ свободно. Позднее этот результат был обобщен Вичем [106] на случай локально компактных групп. Таким образом, дискретная группа $G$ действует непрерывно и свободно на своей стоун-чеховской компактификации $\beta(G)$. Заметим, что действие локально компактной группы на своей стоун-чеховской компактификации уже не всегда непрерывно, по этому поводу см. [78].

Заключим этот пункт результатом [4], описывающим свойства действия группы $\mathscr{J}(\mathbf{k})$ на своей стоун-чеховской компактификации.

Теорема 8.7. а) Для любого дискретного колъца $\mathbf{k}$ действие группь $\mathscr{J}(\mathbf{k})$ на своей компактификации Стоуна-Чеха $\beta(\mathscr{J}(\mathbf{k}))$ является свободным.

b) Если кольцо $\mathbf{k}$ бесконечно и дискретно, то действие группь $\mathscr{J}(\mathbf{k})$ на $\beta(\mathscr{J}(\mathbf{k}))$ разрывно.

\section{Список литературы}

[1] И.В. Артельных, "Произведения Масси и спектральная последовательность Бухштабера", УМH, 55:3(333) (2000), 165-166; англ. пер.: I. V. Artel'nykh, "Massey products and the Bukhshtaber spectral sequence", Russian Math. Surveys, $\mathbf{5 5 : 3}$ (2000), 559-561. 
[2] И. К. Бабенко, С. А. Богатый, "О группе подстановок формальных целочисленных рядов", Изв. РАН. Сер. матем., 72:2 (2008), 39-64; англ. пер.: I. K. Babenko, S.A. Bogatyi, "On the group of substitutions of formal power series with integer coefficients", Izv. Math., 72:2 (2008), 241-264.

[3] I. Babenko, S. Bogatyi, On topological properties of the formal power series substitution group, arXiv: 0912.1813v1.

[4] И. К. Бабенко, С. А. Богатый, "Аменабельность группы подстановок формальных степенных рядов", Изв. РАН. Сер. матем., 75:2 (2011), 19-34; англ. пер.: I. K. Babenko, S. A. Bogatyi, "The amenability of the substitution group of formal power series", Izv. Math., 75:2 (2011), 239-252.

[5] И. К. Бабенко, И. А. Тайманов, "О существовании неформальных односвязных симплектических многообразий", УМН, 53:5(323) (1998), 225-226; англ. пер.: I.K. Babenko, I. A. Taimanov, "On the existence of informal simply connected symplectic manifolds", Russian Math. Surveys, 53:5 (1998), 1082-1083.

[6] И.КК. Бабенко, И. А. Тайманов, "О неформальных односвязных симплектических многообразиях", Сиб. матем. журн., 41:2 (2000), 253-269; англ. пер.: I. K. Babenko, I. A. Taimanov, "On nonformal simply connected symplectic manifolds", Siberian Math. J., 41:2 (2000), 204-217.

[7] И.К. Бабенко, И.А. Тайманов, "Произведения Масси в симплектических многообразиях", Матем. сб., 191:8 (2000), 3-44; англ. пер.: I. K. Babenko, I. A. Taimanov, "Massey products in symplectic manifolds", Sb. Math., 191:7-8 (2000), 1107-1146.

[8] I. K. Babenko, I. A. Taimanov, "On the formality problem for symplectic manifolds", Global differential geometry: the mathematical legacy of Alfred Gray (Bilbao, 2000), Contemp. Math., 288, Amer. Math. Soc., Providence, RI, 2001, 1-9.

[9] I. N. Baker, "Zusammensetzungen ganzer Funktionen", Math. Z., 69:1 (1958), 121-163.

[10] I. N. Baker, "Permutable power series and regular iteration", J. Austral. Math. Soc., 2:3 (1962), 265-294.

[11] Y. Barnea, M. Ershov, T. Weigel, "Abstract commensurators of profinite groups", Trans. Amer. Math. Soc., 363:10 (2011), 5381-5417.

[12] Y. Barnea, B. Klopsch, "Index-subgroups of the Nottingham group", Adv. Math., 180:1 (2003), 187-221.

[13] Y. Barnea, A. Shalev, "Hausdorff dimension, pro- $p$ groups, and Kac-Moody algebras", Trans. Amer. Math. Soc., 349:12 (1997), 5073-5091.

[14] S. I. Bogataya, S. A. Bogatyi, "Series of commutants of the group of substitutions of formal series $\mathscr{J}\left(\mathbb{Z}_{2}\right)$ ", Topology Appl. (to appear).

[15] S. Bogatyi, "On the nonexistence of iterative roots", Topology Appl., 76:2 (1997), 97-123.

[16] Н. Н. Боголюбов, О некоторых эргодических свойствах непрерывных групп преобразований, Н.Н. Боголюбов, Избранные труды, т. 1, Наукова думка, Киев, 1969, 561-569.

[17] А.Д. Брюно, “О сходимости преобразования дифференциальных уравнений к нормальной форме", Докл. АН ССCP, 165 (1965), 987-989; англ. пер.: A.D. Bryuno, "Convergence of transformations of differential equations to normal form", Soviet Math. Dokl., 6 (1965), 1536-1538.

[18] А. Д. Брюно, “Аналитическая форма дифференциальных уравнений. I, II", Тр. ММО, 25, Изд-во Моск. ун-та, М., 1971, 119-262; 26, 1972, 199-239; англ. пер.: A. D. Bryuno, "Analytical form of differential equations. I, II", Trans. Moscow Math. Soc., 1973, 131-288; 1974, 199-239.

[19] В. М. Бухштабер, “Двузначные формальные группы. Алгебраическая теория и приложение к кобордизмам. I", Изв. АН СССР. Сер. матем., 39:5 (1975), 
1044-1064; англ. пер.: V. M. Buhštaber, "Two-valued formal groups. Algebraic theory and applications to cobordism. I", Math. USSR-Izv., 9:5 (1975), 987-1006.

[20] В. М. Бухштабер, "Алгебра Стинрода - обертывающая алгебра супергруппы p-адических диффеоморфизмов прямой”, Добавление к кн.: Н. Стинрод, Д. Эпстейн, Когомологические операции, Наука, М., 1983.

[21] В. М. Бухштабер, "Группы полиномиальных преобразований прямой, неформальные симплектические многообразия и алгебра Ландвебера-Новикова", УМH, 54:4(328) (1999), 161-162; англ. пер.: V.M. Buchstaber, "Groups of polynomial transformations of a line, non-formal symplectic manifolds, and the Landweber-Novikov algebra", Russian Math. Surveys, 54:4 (1999), 837-838.

[22] В. М. Бухштабер, А. В. Шокуров, "Алгебра Ландвебера-Новикова и формальные векторные поля на прямой”, Функи. анализ и его прил., 12:3 (1978), 1-11; англ. пер.: V. M. Buchstaber, A. V. Shokurov, "The Landweber-Novikov algebra and formal vector fields on the line", Funct. Anal. Appl., 12:3 (1978), 159-168.

[23] R. Camina, "Subgroups of the Nottingham group", J. Algebra, 196:1 (1997), 101-113.

[24] R. Camina, "Some natural subgroups of the Nottingham group", Proc. Edinburgh Math. Soc. (2), 42:2 (1999), 333-339.

[25] R. Camina, "The Nottingham group", New horizons in pro-p groups, Progr. Math., 184, eds. M. du Sautoy, D. Segal, A. Shalev, Birkhäuser Boston, Inc., Boston, MA, 2000, 205-221.

[26] J. W. Cannon, W. J. Floyd, W. R. Parry, "Introductory notes on Richard Thompson's groups", Enseign. Math. (2), 42:3-4 (1996), 215-256.

[27] J. Conway, A course in functional analysis, Grad. Texts in Math., 96, Springer-Verlag, New York, 1985, xiv+404 pp.

[28] H. Cremer, "Über die Häufigkeit der Nichtzentren", Math. Ann., 115:1 (1938), $573-580$.

[29] M. M. Day, "Fixed point theorems for compact convex sets", Illinois J. Math., 5 (1961), 585-590.

[30] M. M. Day, "Correction to my paper 'Fixed point theorems for compact convex sets'", Illinois J. Math., 8 (1964), 713.

[31] P. de la Harpe, "Moyennabilité de quelques groupes topologiques de dimension infinie", C. R. Acad. Sci. Paris Sér. A-B, 277 (1973), A1037-A1040.

[32] Ф.А. Гриффитс, П. Делинь, Д. Морган, Д. Сюлливан, "Вещественная гомотопическая теория кэлеровых многообразий", УМH, 32:3(195) (1977), 119-152; пер. с англ.: P. Deligne, P. Griffits, J. Morgan, D. Sullivan, "Real homotopy theory of Kähler manifolds", Invent. Math., 29:3 (1975), 245-274.

[33] M. du Sautoy, I. Fesenko, "Where the wild things are: ramification groups and the Nottingham group", New horizons in pro-p groups, Progr. Math., 184, Birkhäuser Boston, Boston, MA, 2000, 287-328.

[34] J. Dugundji, Topology, Allyn and Bacon, Inc., Boston, MA, 1966, xvi+447 pp.

[35] J. Écalle, "Quelques résultats nouveaux en théorie de la $C$-itération", C. R. Acad. Sci. Paris Sér. A-B, 272 (1971), A225-A227.

[36] J. Écalle, "Nature du groupe des ordres d'itération complexes d'une transformation holomorphe au voisinage d'un point fixe de multiplicateur 1", C. R. Acad. Sci. Paris Sér. A-B, 276 (1973), A261-A263.

[37] J. Écalle, "Invariants holomorphes simples des transformations de multiplicateur 1", C. R. Acad. Sci. Paris Sér. A-B, 276 (1973), A375-A378.

[38] R. Ellis, "Universal minimal sets", Proc. Amer. Math. Soc., 11:4 (1960), 540-543.

[39] D. B. A. Epstein, "Almost all subgroups of a Lie group are free", J. Algebra, 19:2 (1971), 261-262. 
[40] P. Erdős, E. Jabotinsky, "On analytic iteration", J. Anal. Math., 8 (1960/1961), 361-376.

[41] M. Ershov, "New just-infinite pro- $p$ groups of finite width and subgroups of the Nottingham group", J. Algebra, 275:1 (2004), 419-449.

[42] M. Ershov, "The Nottingham group is finitely presented", J. London Math. Soc. (2), 71:2 (2005), 362-378.

[43] M. Ershov, "On subgroups of the Nottingham group of positive Hausdorff dimension", Comm. Algebra, 35:1 (2007), 193-206.

[44] M. Ershov, "On the commensurator of the Nottingham group", Trans. Amer. Math. Soc., 362 (2010), 6663-6678.

[45] B. L. Feigin, D. G. Fuchs, V.S. Retakh, "Massey operations in the cohomology of the infinite-dimensional Lie algebra $L_{1}$ ", Topology and geometry - Rokhlin Seminar, Lecture Notes in Math., 1346, Springer, Berlin, 1988, 13-31.

[46] M. Fernández, M. Gotay, A. Gray, "Compact parallelizable four dimensional symplectic and complex manifolds", Proc. Amer. Math. Soc., 103:4 (1988), 1209-1212.

[47] M. Fernández, A. Gray, J. W. Morgan, "Compact symplectic manifolds with free circle actions, and Massey products", Michigan Math. J., 38:2 (1991), 271-283.

[48] I. Fesenko, "On just infinite pro- $p$-groups and arithmetically profinite extensions of local fields", J. Reine Angew. Math., 517 (1999), 61-80.

[49] Д. Б. Фукс, Когомологии бесконечномерных алгебр Ли, Наука, М., 1984, 272 с.; англ. пер.: D. B. Fuks, Cohomology of infinite-dimensional Lie algebras, Contemp. Soviet Math., Consultants Bureau, New York, 1986, xii+339 pp.

[50] Л.В.Гончарова, "Когомологии алгебр Ли формальных векторных полей на прямой”, Функи. анализ и его прил., 7:2 (1973), 6-14; англ. пер.: L. V. Goncharova, "The cohomologies of Lie algebras of formal vector fields on the line", Funct. Anal. Appl., 7:2 (1973), 91-97.

[51] M. Gotô, "On the group of formal analytic transformations", Kōdai Math. Seminar Reports, 2:3 (1950), 45-46.

[52] M. Goze, E. Remm, "Non existence of complex structures on filiform Lie algebras", Comm. Algebra, 30:8 (2002), 3777-3788.

[53] Ф. Гринлиф, Инвариантные средние на топологических группах и их приложения, Мир, М., 1973, 136 с.; пер. с англ.: F. P. Greenleaf, Invariant means on topological groups and their applications, Van Nostrand Mathematical Studies, 16, Van Nostrand Reinhold Co., New York-Toronto-London, 1969, ix+113 pp.

[54] P. Hegedüs, "The Nottingham group for $p=2$ ", J. Algebra, 246:1 (2001), 55-69.

[55] N. Hindman, D. Strauss, Algebra in the Stone-Čech compactification: Theory and applications, de Gruyter Exp. Math., 27, Walter de Gruyter \& Co., Berlin, 1998, xiv $+485 \mathrm{pp}$.

[56] S.-T. Hu, "Cohomology theory in topological groups", Michigan Math. J., 1:1 (1952), $11-59$.

[57] E. Jabotinsky, "Analytic iteration", Trans. Amer. Math. Soc., 108:3 (1963), 457-477.

[58] E. Jabotinsky, "l-sequences for nonembeddable functions", Proc. Amer. Math. Soc., 17:3 (1966), 738-741.

[59] S.A. Jennings, "Substitution groups of formal power series", Canad. J. Math., 6 (1954), 325-340.

[60] D. L. Johnson, "The group of formal powers series under substitution", J. Austral. Math. Soc. Ser. A, 45:3 (1988), 296-302.

[61] G. Julia, "Sur quelques problèmes rélatifs à l'itération des fractions rationnelles", C. R. Acad. Sci. Paris, 168 (1919), 147-149.

[62] K. Keating, "How close are $p$ th powers in the Nottingham group?", J. Algebra, 287:2 (2005), 294-309. 
[63] А.А. Кириллов, Элементы теории представлений, 2-е изд., Наука, М., 1978, 343 с.; англ. пер. 1-го изд.: А. А. Kirillov, Elements of the theory of representations, Grundlehren Math. Wiss., 220, Springer-Verlag, Berlin-New York, 1976, xi+315 pp.

[64] G. Klaas, C. R. Leedham-Green, W. Plesken, Linear pro-p-groups of finite width, Lecture Notes in Math., 1674, Springer-Verlag, Berlin, 1997, viii+115 pp.

[65] B. Klopsch, "Automorphisms of the Nottingham group", J. Algebra, 223:1 (2000), $37-56$.

[66] B. Klopsch, "Normal subgroups in substitution groups of formal power series", J. Algebra, 228:1 (2000), 91-106.

[67] K. Kodaira, "On the structure of compact complex analytic surfaces. I", Amer. J. Math., 86:4 (1964), 751-798.

[68] G. Koenigs, "Recherches sur les intégrales de certaines équations fonctionnelles", Ann. Sci. Ecole Norm. Sup. (3), 3 (1884), 3-41.

[69] D. Kraines, "Massey higher products", Trans. Amer. Math. Soc., 124:3 (1966), 431-449.

[70] P. S. Landweber, "Cobordism operations and Hopf algebras", Trans. of Amer. Math. Soc., 129:1 (1967), 94-110.

[71] C. R. Leedham-Green, S. McKay, The structure of groups of prime power order, London Math. Soc. Monogr. (N.S.), 27, Oxford Univ. Press, Oxford, 2002, xii+334 pp.

[72] A. Lubotzky, J.S. Wilson, "An embedding theorem for profinite groups", Arch. Math. (Basel), 42:5 (1984), 397-399.

[73] Б. Я. Левин, Распределение корней цельх функиий, ГИТТЛ, М., 1956, 632 с.; англ. пер.: В. Ya. Levin, Distribution of zeros of entire functions, Amer. Math. Soc., Providence, RI, 1964, viii+493 pp.

[74] А. И. Мальцев, "Об одном классе однородных пространств", Изв. АН СССР. Сер. матем., 13:1 (1949), 9-32; англ. пер.: A. I. Malcev, On a class of homogeneous spaces, Amer. Math. Soc. Transl., 1951, № 39, 1951, 33 pp.

[75] А.И. Мальцев, "Нильпотентные группы без кручения", Изв. АН СССР. Сер. матем., 13:3 (1949), 201-212.

[76] J.P. May, "Matric Massey products", J. Algebra, 12:4 (1969), 533-568.

[77] М.Г. Мегрелишвили, "Об эквивариантной нормальности”, Сообщ. АН ГССР, 111:1 (1983), 17-19.

[78] М. Г. Мегрелишвили, "Тихоновское $G$-пространство, не обладающее бикомпактным $G$-расширением и $G$-линеаризацией”, УМH, 43:2(260) (1988), 145-146; англ. пер.: M. G. Megrelishvili, "A Tikhonov $G$-space not admitting a compact Hausdorff G-extension or G-linearization", Russian Math. Surveys, 43:2 (1988), 177-178.

[79] Д. В. Миллионщиков, “Алгебра формальных векторных полей на прямой и гипотеза Бухштабера", Функи. анализ и его прил., 43:4 (2009), 26-44; англ. пер.: D. V. Millionshchikov, "Algebra of formal vector fields on the line and Buchstaber's conjecture", Funct. Anal. Appl., 43:4 (2009), 264-278.

[80] D. V. Millionschikov, "Cohomology of nilmanifolds and Gontcharova's theorem" (Bilbao, 2000), Contemp. Math., 288 (2001), 381-385.

[81] J. Milnor, "Remarks on infinite-dimensional Lie groups", Relativity, groups and topology, v. II (Les Houches, 1983), North-Holland, Amsterdam, 1984, 1007-1057.

[82] B. Muckenhoupt, "Automorphisms of formal power series under substitution", Trans. Amer. Math. Soc., 99:3 (1961), 373-383.

[83] B. Muckenhoupt, "Some results on analytic iteration and conjugacy", Amer. J. Math., 84:1 (1962), 161-169.

[84] B. H. Neumann, H. Neumann, "Embedding theorems for groups", J. London Math. Soc., 34 (1959), 465-479. 
[85] K. Nomizu, "On the cohomology of homogeneous spaces of nilpotent Lie groups", Ann. of Math. (2), 59 (1954), 531-538.

[86] С.П. Новиков, "Методы алгебраической топологии с точки зрения теории кобордизмов", Изв. АН СССР. Сер. матем., 31:4 (1967), 855-951; англ. пер.: S.P. Novikov, "The methods of algebraic topology from the viewpoint of cobordism theory", Math. USSR-Izv., 1:4 (1967), 827-913.

[87] P. P. Pálfy, "The number of conjugacy classes in some quotients of the Nottingham group", Proc. Edinburgh Math. Soc. (2), 41:2 (1998), 369-384.

[88] K. R. Parthasarathy, Probability mesures on metric spaces, Probab. Math. Statist., 3, Academic Press, Inc., New York-London, 1967, xi+276 pp.

[89] A. L. T. Paterson, Amenability, Math. Surveys Monogr., 29, Amer. Math. Soc., Providence, RI, 1988, xx+452 pp.

[90] R. Pérez-Marco, "Solution complète au problème de Siegel de linéarisation d'une application holomorphe au voisinage d'un point fixe (d'après J.-C. Yoccoz)", Séminaire Bourbaki, v. 1991/92, Astérisque, 206, Soc. Math. France, Paris, 1992, 273-310 (exp. $753)$.

[91] G. A. Pfeiffer, "On the conformal mapping of curvilinear angles. The functional equation $\phi[f(x)]=a_{1} \phi(x)$ ", Trans. Amer. Math. Soc., 18:2 (1917), 185-198.

[92] G. Pólya, G. Szegö, Problems and theorems in analysis, v.2: Theory of functions, zeros, polynomials, determinants, number theory, geometry, Revised and enlarged translation of the 4th German edition, Grundlehren Math. Wiss., 216, Springer-Verlag, New York-Heidelberg-Berlin, 1976, xi+392 pp.

[93] N.W. Rickert, "Amenable groups and groups with the fixed point property", Trans. Amer. Math. Soc., 127:2 (1967), 221-232.

[94] P. Samuel, "Ultrafilters and compactifications of uniform spaces", Trans. Amer. Math. Soc., 64:1 (1948), 100-132.

[95] E. Schröder, "Ueber iterirte Functionen", Math. Ann., 3:2 (1870), 296-322.

[96] J.-P. Serre, Cohomologie galoisienne, Troisième édition, Lecture Notes in Math., 5, Springer-Verlag, Berlin-New York, 1965, v+212 pp.; рус. пер. 2-го издания: Ж. П. Серр, Когомологии Галуа, Мир, М., 1968, 208 с.

[97] A. Shalev, "Some problems and results in the theory of pro- $p$ groups", Groups'93 Galway/St. Andrews, v. 2, London Math. Soc. Lecture Note Ser., 212, Cambridge Univ. Press, Cambridge, 1995, 528-542.

[98] A. Shalev, "Lie methods in the theory of pro- $p$ groups", New horizons in pro- $p$ groups, Progr. Math., 184, Birkhäuser Boston, Boston, MA, 2000, 1-54.

[99] C. L. Siegel, "Iteration of analytic functions", Ann. of Math. (2), 43:4 (1942), 607-612.

[100] Э. Спеньер, Алгебрачческая топология, Мир, М., 1971, 680 с.; пер. с англ.: E. H. Spanier, Algebraic topology, McGraw-Hill, New York-Toronto-London, 1966, xiv $+528 \mathrm{pp}$.

[101] Н. Стинрод, Д. Эпстейн, Когомологические операции, Наука, М., 1983, 232 с.; пер. с англ.: N.E. Steenrod, Cohomology operations, Lectures. Written and revised by D. B. A. Epstein, Ann. of Math. Stud., 50, Princeton Univ. Press, Princeton, NJ, 1962, vii+139 pp.

[102] S. Sternberg, "On the structure of local homeomorphisms of Euclidean $n$-space, II", Amer. J. Math., 80:3 (1958), 623-631.

[103] Р. Стонг, Заметки по теории кобордизмов, Мир, М., 1973, 372 с.; пер. с англ.: R. Stong, Notes on cobordism theory, Math. Notes, Princeton Univ. Press, Princeton, NJ; Univ. of Tokyo Press, Tokyo, 1968, v+354+lvi pp.

[104] B. Szegedy, "Almost all finitely generated subgroups of the Nottingham group are free", Bull. London Math. Soc., 37:1 (2005), 75-79. 
[105] G. Szekeres, "Fractional iteration of entire and rational functions", J. Austral. Math. Soc., 4:2 (1964), 129-142.

[106] W. A. Veech, "Topological dynamics", Bull. Amer. Math. Soc., 83:5 (1977), 775-830.

[107] J. von Neumann, "Zur allgemeinen Theorie des Masses", Fundamenta, 13 (1929), $73-116$.

[108] С. М. Воронин, "Аналитическая классификация ростков конформных отображений $(\mathbb{C}, 0) \rightarrow(\mathbb{C}, 0)$ с тождественной линейной частью", Функи. анализ и его прил., 15:1 (1981), 1-17; англ. пер.: S. M. Voronin, "Analytic classification of germs of conformal mappings $(\mathbb{C}, 0) \rightarrow(\mathbb{C}, 0)$ with identity linear part", Funct. Anal. Appl., 15:1 (1981), 1-13.

[109] J.S. Wilson, "Embedding theorems for residually finite groups", Math. Z., 174:2 (1980), 149-157.

[110] J. S. Wilson, Profinite groups, London Math. Soc. Monogr. (N.S.), 19, Oxford Univ. Press, New York, 1998, xii+284 pp.

[111] J.-P. Wintenberger, "Extensions abéliennes et groupes d'auto-morphismes de corps locaux", C. R. Acad. Sci. Paris Sér. A-B, 290:5 (1980), A201-A203.

[112] E. Witt, "Konstruktion von galoisschen Körpern der Charakteristik $p$ zu vorgegebener Gruppe der Ordnung $p^{f} "$, J. Reine Angew. Math., 174 (1936), 237-245.

[113] J.-C. Yoccoz, "Théorème de Siegel, nombres de Bruno et polynômes quadratiques", Petits diviseurs en dimension 1, Astérisque, 231, Soc. Math. France, Paris, 1995, 3-8.

[114] J.-C. Yoccoz, "Linéarisation des germes de difféomorphismes holomorphes de (C, 0)", C. R. Acad. Sci. Paris Sér. I Math., 306:1 (1988), 55-58.

[115] I. O. York, "The exponent of certain finite p-groups", Proc. Edinburgh Math. Soc. (2), 33:3 (1990), 483-490.

И. К. Бабенко (I. K. Babenko)

Université Montpellier II, Montpellier, France;

Московский государственный университет

им. М. В. Ломоносова

E-mail: babenko@math.univ-montp2.fr
Поступила в редакцию

28.02 .2012 